\title{
MEDIDAS REPETIDAS CATEGORIZADAS INCOMPLETAS: UMA APLICAÇÃO À CONFIABILIDADE DE CULTIVARES
}

\author{
FRANCISCO DAS CHAGAS ESTEVAM DA FONSECA
}

Engenheiro Agrônomo

Orientador: Prof. Dr. ANTONIO FRANCISCO IEMMA

Tese apresentada à Escola Superior de Agricultura "Luiz de Queiroz", Universidade de São Paulo, para obtenção do título de Doutor em Agronomia. Área de Concentração: Estatística e Experimentação Agronômica.

PIRACICABA

Estado de São Paulo - Brasil

Fevereiro - 1999 
Dados Internacionais de Catalogação na Publicação (CIP) DIVISÃO DE BIBLIOTECA E DOCUMENTAÇÃO - Campus "Luiz de Oueiroz"/USP

Fonseca, Francisco das Chagas Estevam da Medidas repetidas categorizadas incompletas: uma aplicaçăo à confiabilidade de cultivares / Francisco das Chagas Estevam da Fonseca. - - Piracicaba, 1999.

$77 \mathrm{p}$.

Tese (doutorado) - Escola Superior de Agricultura Luiz de Queiroz, 1999. Bibliografia.

1. Confiabilidade 2. Estatistica aplicada 3. Método estatistico 4. Variedade I. Título

CDD 519.5 


\section{AGRADECIMENTOS}

Ao Prof. Dr. Antonio Francisco Iemma, pela amizade, pela atenciosidade e pela orientação segura durante todo o transcorrer do Curso;

À Universidade Federal do Rio Grande do Norte, pela oportunidade de aperfeiçoamento;

À CAPES/PICD, pela bolsa concedida;

Aos Professores do Departamento de Matemática e Estatística da Escola Superior de Agricultura "Luiz de Queiroz", pela cordialidade e pelos ensinamentos recebidos;

Aos Pós-graduandos do Curso de Estatística e Experimentação Agronômica do Departamento de Matemática e Estatística, pelo companheirismo e pelos incentivos nas horas mais dificeis;

Aos Funcionários do Departamento de Matemática e Estatística da Escola Superior de Agricultura "Luiz de Queiroz", pela maneira fraterna e prestativa com que me trataram;

Aos companheiros de Natal, por me estimularem a fazer o Curso;

Aos companheiros de Piracicaba, por me ajudarem a afastar a solidão; 


\section{DEDICATÓRIA}

Ao meu pai, Chicola.

Aos meus irmãos Antônio, Zé Antônio, Nadinha e Claudinha.

À minha esposa, Carlinda (Nêga).

Aos meus filhos Neluza (Babuba), Daniel (Dedé) e Daliana (Pixotota).

Ao "velho" Manuel Rosa, por me deixar ficar sob a sombra do "seu" juazeiro e ouvir um pouco da sua sabedoria simples.

Ao meu avô, Chico Elesbão, por ter, algumas vezes, "fingido" ser criança quando eu é que o era.

Ao meu sogro, "seo" João de Bento.

À minha sogra, dona Neuza.

Ao meu cunhado, Joãozinho.

Ao meu irmão, Giovani.

À minha irmã, Lucinha.

À minha mãe, LUiZa, que mesmo brilhando em outra dimensão, continua a alumiar o meu caminho.

(Já encantados) 


\section{SUMÁRIO}

\section{Página}

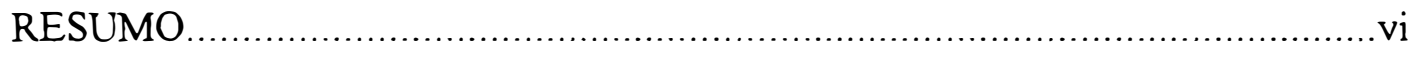

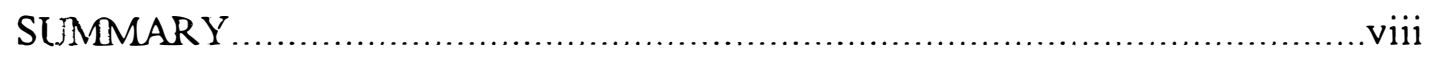

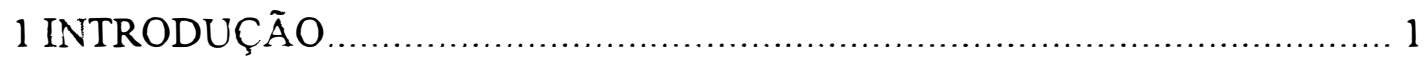

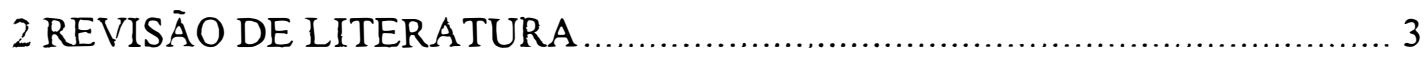

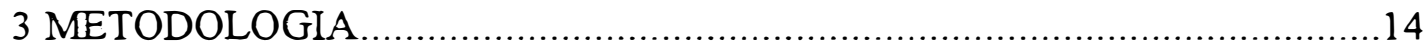

3.10 procedimento CATMOD: PROC CATMOD (SAS, 1990) ....................17

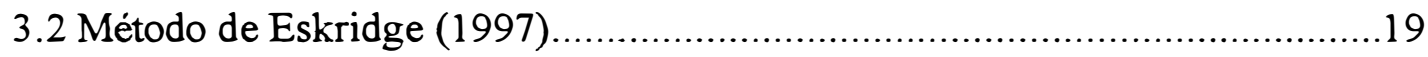

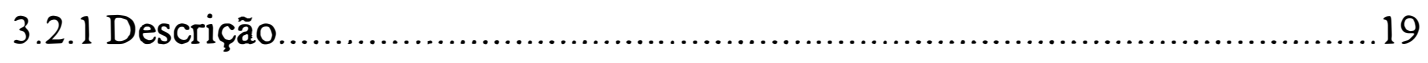

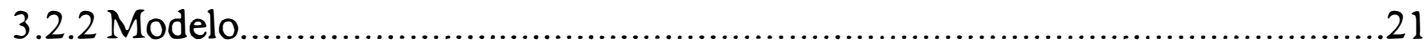

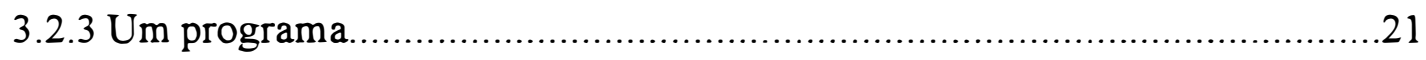

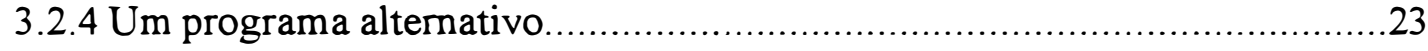

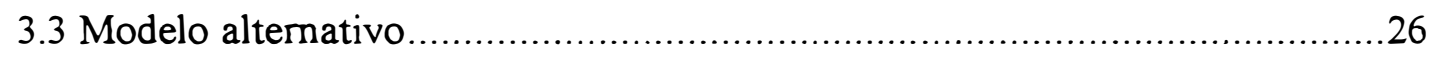

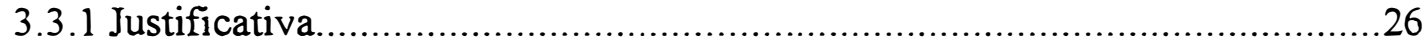

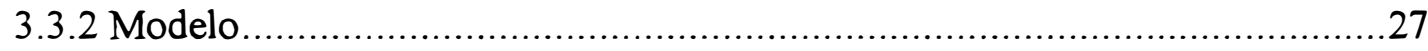

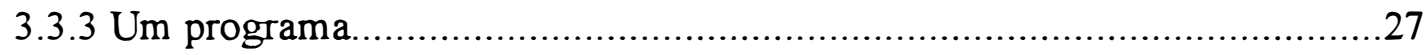

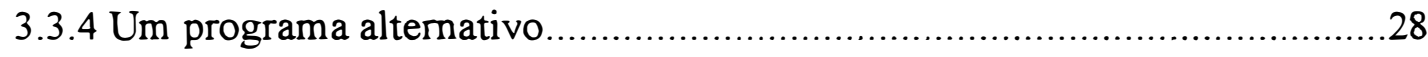

3.4 Teste do mecanismo de dados incompletos (Park \& Davis, 1993) ................. 31

3.4.1 A estatística de Wald para homogeneidade de parâmetros específicos

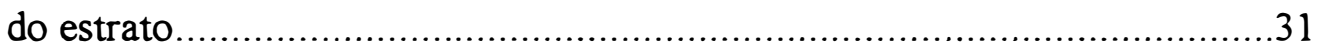

3.4.2 A estatística de Wald para o mecanismo de dados incompletos.................32

3.4.3 Teste do mecanismo de dados incompletos usando anos no modelo...........34

3.4.4 Teste do mecanismo de dados incompletos usando locais no modelo.........38

4 RESULTADOS E DISCUSSÃO ............................................... 41

4.1 Primeiro conjunto de dados..................................................... 41

4.1.1 Considerações iniciais.......................................................... 41 


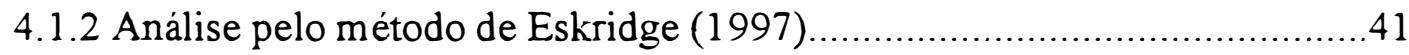

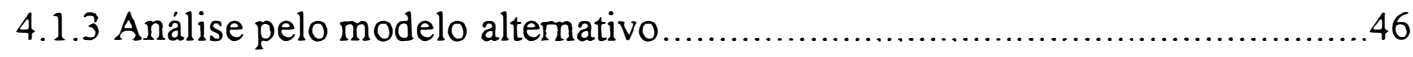

4.1.4 Teste do mecanismo de dados incompletos ........................................50

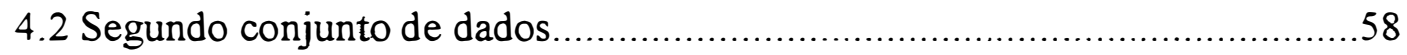

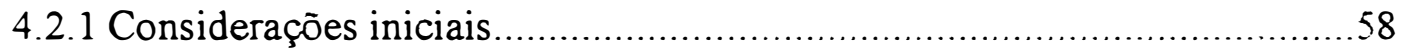

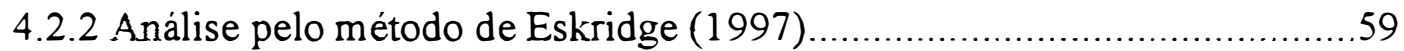

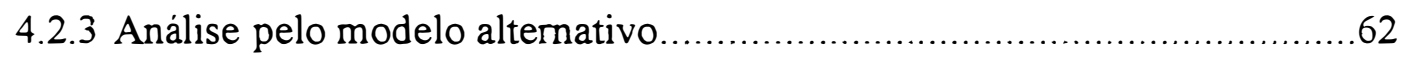

4.2.4 Teste do mecanismo de dados incompletos .........................................6

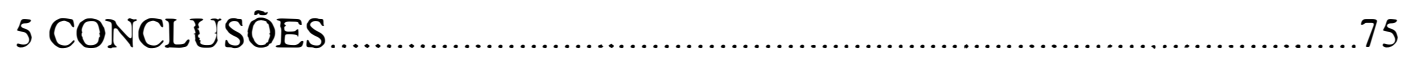

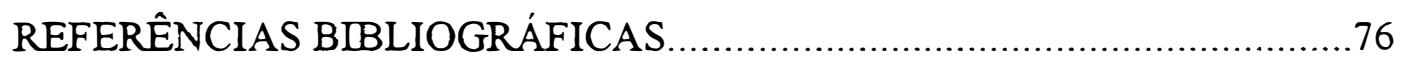




\title{
MEDIDAS REPETIDAS CATEGORIZADAS INCOMPLETAS: UMA APLICAÇÃO À CONFIABILIDADE DE CULTIVARES
}

\author{
Autor: FRANCISCO DAS CHAGAS ESTEVAM DA FONSECA \\ Orientador: Prof. Dr. ANTONIO FRANCISCO IEMMA
}

\section{RESUMO}

Experimentos com medidas repetidas são aqueles nos quais cada individuo é observado em diversos tempos diferentes ou sob condições experimentais diferentes. As medidas repetidas são denominadas categorizadas quando a variável resposta é representada por categorias de respostas.

Em muitas situações ocorre a existência de dados incompletos.

Um dos métodos mais utilizados para a análise de dados incompletos é o de Woolson \& Clarke (1984), que assume que os dados são "incompletos por razões totalmente aleatórias" (MCAR - "missing completely at random"), conforme definido em Little \& Rubin (1987).

Uma aplicação agronômica importante de medidas repetidas categorizadas com dados incompletos foi apresentada por Eskridge (1997). O autor utilizou o conceito de confiabilidade e o método de Woolson \& Clarke (1984) para avaliar e comparar hibridos de milho, provenientes de um programa de melhoramento genético.

O objetivo do presente trabalho foi aplicar o método de Eskridge (1997), em dois conjuntos de dados, propor um modelo alternativo àquele usado pelo autor, bem como verificar, em cada caso, a suposição de MCAR, utilizando-se o método de Park \& Davis (1993).

Após as análises, concluiu-se que: 
- em alguns casos o modelo alternativo pode ser mais informativo que o modelo de Eskridge (1997);

- o teste do mecanismo de dados incompletos de Park \& Davis (1993) é dependente do modelo, não analisando a estrutura, propriamente dita, dos dados. 


\title{
REPEATED CATEGORICAL INCOMPLETE MEASUREMENTS: AN APPLICATION TO RELIABILITY OF VARIETIES
}

\author{
Author: FRANCISCO DAS CHAGAS ESTEVAM DA FONSECA \\ Adviser: Prof. Dr. ANTONIO FRANCISCO IEMMA
}

\section{SUMMARY}

Repeated measures studies are that which each subject is observed in many different times or under different experimentals conditions. The repeateds measurements are named categorical when the response variable is represented by categories of response.

In much situations occur a existency of missing data.

One of the methods more utilized for the analysis of missing data is from the Woolson \& Clarke (1984), that assumes the datas are missing completely at random (MCAR), according determined in Little \& Rubin (1987).

An important agronomic application of repeated categorical measurements with missing data was shown by Eskridge (1997). The author utilized the conceit of reliability and the method of Woolson \& Clarke (1984) to evaluate and to compare hybrid of com, originating of genetic breeding program.

The objective of the present work was to apply the method of Eskridge (1997), in two sets of data, to propose a alternative model from that used by author, also to verify in each case, the hypothesis of MCAR, utilizing o method of Park \& Davis (1993).

Thereafter the analysis, was concluded that::

- in some cases the alternative model can be more informative than the model of Eskridge (1997); 
- the test of the missing data mechanism of Park \& Davis (1993) is dependent of the model, does not analysing the struture of the data. 


\section{INTRODUÇÃO}

Experimentos com medidas repetidas são aqueles nos quais cada indivíduo é observado em diversos tempos diferentes ou sob condições experimentais diferentes. As medidas repetidas são denominadas categorizadas quando a variável resposta é representada por categorias de respostas.

A análise de dados completos é realizada, sem maiores dificuldades, utilizando-se, por exemplo, o método de mínimos quadrados ponderados proposto por Grizzle, Starmer \& Koch (1969), denominado, posteriormente, método GSK.

Uma forma, simplista e cômoda, de analisarem-se experimentos com dados incompletos é, simplesmente, ignorar-se a parte correspondente aos dados incompletos, efetuando-se a análise somente na parte referente aos dados completos. Evidentemente, essa é uma maneira pouco eficiente de análise de dados.

Desta forma, torna-se de grande importância o conhecimento de métodos que incorporem os dados incompletos à análise, tais como aqueles apresentados por Koch et al. (1972), denotado por KO; Woolson \& Clarke (1984), denotado por WC. Estes métodos assumem que os dados são "incompletos por razões totalmente aleatórias" (MCAR - "missing completely at random"), conforme definido em Little \& Rubin (1987).

Uma aplicação agronômica importante de medidas repetidas categorizadas com dados incompletos foi apresentada por Eskridge (1997). O autor utilizou o conceito de confiabilidade e o método WC para avaliar e comparar híbridos de milho, provenientes de um programa de melhoramento genético. 
Ressalte-se que o autor utilizou o método WC sem testar a condição de MCAR dos dados

O objetivo do presente trabalho é aplicar o método de Eskridge (1997), em dois conjuntos de dados, propor um modelo alternativo àquele usado pelo autor, bem como verificar, em cada caso, a suposição de MCAR, através do método descrito em Park \& Davis (1993). Para tanto, são implementados programas utilizando-se o Procedimento CATMOD (CATegorical data MODeling) do SAS (Statistical Analysis System), conforme SAS (1990). 


\section{REVISĀO DE LITERATURA}

Grizzle, Starmer \& Koch (1969) desenvolveram um método pioneiro para a análise de dados categorizados através de modelos lineares, utilizando mínimos quadrados ponderados.

Com base neste trabalho, diversos outros métodos, utilizando mínimos quadrados ponderados, surgiram para lidar com situações específicas envolvendo dados categorizados, tais como dados incompletos e medidas repetidas. Posteriormente, este método, que é, na sua forma original, utilizado apenas para dados completos, ficou conhecido como GSK.

Para o desenvolvimento do método, os autores consideram os dados hipotéticos apresentados na Tabela 1 e as probabilidades esperadas das caselas apresentadas na Tabela 2 .

Tabela 1. Tabela de contingência dos dados observados

\begin{tabular}{cccccc}
\hline \multicolumn{7}{c}{ Categorias de respostas } & & \\
\hline Populações & 1 & 2 & $\ldots$ & $\mathrm{r}$ & Totais \\
\hline 1 & $\mathrm{n}_{11}$ & $\mathrm{n}_{12}$ & $\ldots$ & $\mathrm{n}_{1 \mathrm{r}}$ & $\mathrm{n}_{1 .}$ \\
2 & $\mathrm{n}_{21}$ & $\mathrm{n}_{22}$ & $\ldots$ & $\mathrm{n}_{2 \mathrm{r}}$ & $\mathrm{n}_{2 .}$ \\
$\cdot$ & $\cdot$ &. & $\ldots$ &. &. \\
$\cdot$ &. &. & $\ldots$ &. &. \\
$\cdot$ & $\cdot$ &. & $\ldots$ &. &. \\
$\mathrm{s}$ & $\mathrm{n}_{\mathrm{s} 1}$ & $\mathrm{n}_{\mathrm{s} 2}$ & $\ldots$ & $\mathrm{n}_{\mathrm{sr}}$ & $\mathrm{n}_{\mathrm{s} .}$ \\
\hline
\end{tabular}


Tabela 2. Tabela de contingência das probabilidades esperadas das caselas

\begin{tabular}{|c|c|c|c|c|c|}
\hline \multicolumn{4}{|c|}{ Categorias de respostas } & \multirow[b]{2}{*}{$\mathrm{r}$} & \multirow[b]{2}{*}{ Totais } \\
\hline Populações & 1 & 2 & $\ldots$ & & \\
\hline 1 & $\pi_{11}$ & $\pi_{12}$ & . & $\pi_{1 \mathrm{r}}$ & 1 \\
\hline 2 & $\pi_{21}$ & $\pi_{22}$ & $\cdots$ & $\pi_{2 r}$ & 1 \\
\hline . & . & . & $\cdots$ & . & . \\
\hline . & . & . & $\cdots$ & $\cdot$ & \\
\hline . & . & . & $\cdots$ & & . \\
\hline s & $\pi_{\mathrm{s} 1}$ & $\pi_{\mathrm{s} 2}$ & $\cdots$ & $\pi_{\mathrm{s} T}$ & 1 \\
\hline
\end{tabular}

E definem:

$\pi_{\mathrm{i}}{ }^{\prime}=\left[\pi_{\mathrm{i} 1}, \pi_{\mathrm{i} 2}, \ldots, \pi_{\mathrm{ir}}\right]=$ vetor $1 \times \mathrm{r}$ das probabilidades de uma resposta $\mathrm{j}$, na $\mathrm{i}$-ésima população;

$\pi^{\prime}=\left[\pi_{1}^{\prime}, \pi_{2}^{\prime}, \ldots, \pi_{\mathrm{s}}^{\prime}\right]=$ vetor composto $1 \times$ rs das probabilidades das caselas;

$\mathrm{p}_{\mathrm{ij}}=\mathrm{n}_{\mathrm{ij}} / \mathrm{n}_{\mathrm{i} \text {. }}=$ proporção observada de uma resposta $\mathrm{j}$, na i-ésima população;

$\mathrm{p}_{\mathrm{i}}^{\prime}=\left[\mathrm{p}_{\mathrm{i} 1}, \mathrm{p}_{\mathrm{i} 2}, \ldots, \mathrm{p}_{\mathrm{ir}}\right]=$ vetor $1 \times \mathrm{r}$ das proporções observadas, na i-ésima população;

$p^{\prime}=\left[p_{1}^{\prime}, p_{2}^{\prime}, \ldots, p_{s}^{\prime}\right]=$ vetor composto $1 \times$ rs das proporções observadas das caselas;

Ademais,

$$
\operatorname{var}\left(\mathrm{p}_{\mathrm{i}}\right)=\mathrm{V}\left(\pi_{\mathrm{i}}\right)=\frac{1}{n_{i .}}\left[\begin{array}{cccc}
\pi_{i 1}\left(1-\pi_{i 1}\right) & -\pi_{i 1} \pi_{i 2} & \ldots & -\pi_{i 1} \pi_{i r} \\
-\pi_{i 2} \pi_{i 1} & \pi_{i 2}\left(1-\pi_{i 2}\right) & \ldots & -\pi_{i 2} \pi_{i r} \\
\ldots & \cdots & \cdots & \ldots \\
-\pi_{i r} \pi_{i 1} & -\pi_{i r} \pi_{i 2} & \cdots & \pi_{i r}\left(1-\pi_{i r}\right)
\end{array}\right],
$$

onde:

$\mathrm{V}\left(\mathrm{p}_{\mathrm{i}}\right)=$ matriz $\mathrm{r} \times \mathrm{r}$ da estimativa amostral de $\mathrm{V}\left(\pi_{\mathrm{i}}\right)$;

$\mathrm{V}(\mathrm{p})=$ matriz bloco diagonal rs $\mathrm{x}$ rs, tendo $\mathrm{V}\left(\mathrm{p}_{\mathrm{i}}\right)$ na diagonal principal;

$f_{m}(\pi)=$ qualquer função dos elementos de $\pi$ que tem derivadas parciais até a segunda ordem em relação aos $\pi_{\mathrm{ij}}, \mathrm{m}=1,2, \ldots, \mathrm{u} \leq(\mathrm{r}-1) \mathrm{s}$;

$f_{m}(p)=f_{m}(\pi)$ avaliada em $\pi=p$; 
$f_{m}(p)=$ qualquer função dos elementos de $p$ que descrevem, em algum aspecto, a relação entre a distribuição das respostas e a natureza das populações;

$[F(\pi)]^{\prime}=\left[f_{1}(\pi), f_{2}(\pi), \ldots, f_{u}(\pi)\right]=$ vetor $1 \times$ u que representa um conjunto de $u$ funções de $\pi$;

$F^{\prime}=[F(p)]^{\prime}=\left[f_{1}(p), f_{2}(p), \ldots, f_{u}(p)\right]=$ vetor $1 \times$ u que representa um conjunto de $u$ funções de $\mathrm{p}$;

$\mathrm{H}=\left[\frac{\partial f_{m}(\pi)}{\partial \pi_{i j}} \mid \pi_{i j}=p_{i j}\right]=$ matriz u $\mathrm{x}$ rs das primeiras derivadas parciais das

funções de $F$, avaliada em $p$;

$\mathrm{S}=\mathrm{HV}(\mathrm{p}) \mathrm{H}^{\prime}$

A matriz $\mathrm{S}$, de dimensão ( $\mathrm{u} \times \mathrm{u}$ ), é a estimativa amostral da matriz de covariâncias de F. Quando $f_{m}(p)$ é uma função linear dos elementos de $p, S$ é a matriz de covariâncias exata de $F$; quando $f_{m}(p)$ é uma função não linear dos elementos de $p$, $S$ é a matriz de covariâncias assintóticas de F, que é obtida pelo método "delta". Uma explanação sobre o método "delta" encontra-se em Agresti (1990).

Admitem, ainda, que $F(\pi)=X \beta$, onde $F(\pi)$ é um vetor de dimensão ( $u x$ 1), $X$ é uma matriz conhecida de posto $v \leq u$, de dimensão ( $u x v$ ), e, $\beta$ é um vetor de parâmetros desconhecidos, de dimensão ( $v$ x 1).

Se o modelo hipotético ajusta-se aos dados, a melhor estimativa normal assintótica (BAN - "best asymptotic normal") de $\beta$ é dada por $b$, onde b é o vetor que minimiza $(\mathrm{F}-\mathrm{Xb}){ }^{\prime} \mathrm{S}^{-1}(\mathrm{~F}-\mathrm{X} \beta)$. O valor minimo dessa forma quadrática pode ser usado para testar o ajuste do modelo $F(\pi)=X \beta$. Admitindo que o modelo presumido proporciona um ajuste adequado aos dados, os autores mostram que um teste da hipótese Ho : $C \beta=0$, é produzido por métodos convencionais de regressão múltipla ponderada, 
onde $\mathrm{C}$ é uma matriz de dimensão $(\mathrm{d} \times \mathrm{v})$, de constantes arbitrárias, de posto linha completo $\mathrm{d} \leq \mathrm{v}$.

A estatística para testar o ajuste do modelo é

$$
\operatorname{SS}[F(\pi)=X \beta]=F^{\prime} S^{-1}-b^{\prime}\left(X^{\prime} S^{-1} X\right) b
$$

que tem uma distribuição qui-quadrado assintoticamente central com $u-v$ graus de liberdade, se o modelo ajusta-se, onde $b=\left(X^{\prime} S^{-1} X\right)^{-1} X^{\prime} S^{-1} F$. Admitindo o modelo, $o$ teste da hipótese $\mathrm{H}_{0}: \mathrm{C} \beta=0$ é produzido por

$$
\mathrm{SS}[\mathrm{C} \beta=0]=\mathrm{b}^{\prime} \mathrm{C}^{\prime}\left[\mathrm{C}\left(\mathrm{X}^{\prime} \mathrm{S}^{-1} \mathrm{X}\right)^{-1} \mathrm{C}^{\prime}\right]^{-1} \mathrm{Cb}
$$

que tem, assintoticamente, uma distribuição qui-quadrado com d graus de liberdade, se $\mathrm{H}_{0}$ é verdadeira.

Koch et al. (1972) estenderam o método GSK para envolver situações com dados incompletos. $\mathrm{O}$ método $\mathrm{KO}$ é bastante similar ao método GSK, utilizado para dados completos. O método GSK efetua a análise de dados categorizados através de modelos lineares, utilizando minimos quadrados ponderados. $\mathrm{O}$ método $\mathrm{KO}$ assume que há s populações de elementos, das quais são selecionadas amostras aleatórias independentes, de tamanhos fixados $n_{1}, n_{2}, \ldots, n_{s}$, respectivamente. As respostas dos $n_{i}$ elementos da i-ésima população são classificados em $r_{i}$ categorias, com $n_{i j}$, onde $j=1,2$, . . . , $\mathrm{r}_{\mathrm{i}}$, indicando o número de elementos classificados na $\mathrm{j}$-ésima categoria para a $\mathrm{i}$ ésima população. Esta estrutura é diferente daquela considerada pelo método GSK, porque ela permite a definição de categorias de respostas e de seu número, $r_{i}$, que pode variar de uma população para outra.

O vetor $\mathbf{n}_{\mathrm{i}}$, onde $\mathbf{n}_{\mathrm{i}}{ }^{\prime}=\left(\mathrm{n}_{\mathrm{i} 1}, \mathrm{n}_{\mathrm{i} 2}, \ldots, \mathrm{n}_{\mathrm{ir}}\right)$, é assumido com uma distribuição multinomial de parâmetros $n_{j}$ e $\pi_{j}$, onde $\pi_{i j}$ é a probabilidade da resposta j na população i. 
Sejam $\mathbf{p}_{\mathrm{i}}=\left(\mathbf{n}_{\mathrm{i}} / \mathbf{n}_{\mathrm{i}}\right)$ e $\mathbf{p}_{\mathrm{g}}$ o vetor composto definido por $\mathbf{p}_{\mathrm{g}}{ }^{\prime}=\left(\mathbf{p}_{1}{ }^{\prime}, \mathbf{p}_{2}{ }^{\prime}, \ldots\right.$, $\left.\mathbf{p}_{\mathrm{s}}{ }^{\prime}\right)$. Uma estimativa consistente para a matriz de covariâncias de $\mathbf{p}_{\mathrm{g}}$ é dada pela matriz bloco diagonal $\mathbf{V}\left(\mathbf{p}_{\mathrm{g}}\right)$, com as matrizes $\mathbf{V}_{\mathrm{i}}\left(\mathbf{p}_{\mathrm{i}}\right)=\left(1 / \mathrm{n}_{\mathrm{i}}\right)\left\{\mathbf{D}_{\mathrm{pi}}-\mathbf{p}_{\mathbf{i}} \mathbf{p}_{\mathrm{i}}{ }^{\prime}\right\}, \mathrm{i}=1,2, \ldots, \mathrm{s}$, dispostas na diagonal principal. Aqui, $\mathbf{D}_{\mathrm{pi}}$ é uma matriz diagonal com elementos do vetor $\mathbf{p}_{\mathrm{i}}$ na diagonal principal.

Seja $F_{1}\left(\mathbf{p}_{\mathrm{g}}\right), \mathrm{F}_{2}\left(\mathbf{p}_{\mathrm{g}}\right), \ldots, \mathrm{F}_{\mathrm{u}}\left(\mathbf{p}_{\mathrm{g}}\right)$ um conjunto de u funções de $\mathbf{p}_{\mathrm{g}}$, cada uma com derivadas parciais até a segunda ordem, em relação aos elementos de $\mathbf{p}_{g}$, presentes em uma região contendo $\pi_{\mathrm{g}}=\mathrm{E}\left\{\mathbf{p}_{\mathrm{g}}\right\}$, onde $\pi_{\mathrm{g}}{ }^{\prime}=\left(\pi_{1}{ }^{\prime}, \pi_{2}{ }^{\prime}, \ldots, \pi_{\mathrm{s}}{ }^{\prime}\right)$. Se $\mathbf{F} \equiv$ $\mathbf{F}\left(\mathbf{p}_{\mathrm{g}}\right)$ é definido por $\left.\mathbf{F}^{\prime} \equiv \mathbf{F}^{\prime}\left(\mathbf{p}_{\mathrm{g}}\right)=\left(\mathrm{F}_{1}\left(\mathbf{p}_{\mathrm{g}}\right), \mathrm{F}_{2}\left(\mathbf{p}_{\mathrm{g}}\right), \ldots, \mathrm{F}_{\mathrm{u}} \mathbf{p}_{\mathrm{g}}\right)\right)$, então a matriz de covariâncias de $\mathbf{F}$ pode ser consistentemente estimada por $\mathbf{V}_{\mathbf{F}}=\mathbf{H}\left[\mathbf{V}\left(\mathbf{p}_{\mathrm{g}}\right)\right] \mathbf{H}^{\prime}$, onde $\mathbf{H}=$ $\left[\mathbf{d F}(\mathbf{x}) / \mathbf{d x} \mid \mathbf{x}=\mathbf{p}_{\mathrm{g}}\right]$. Em todas as aplicações, as funções em $\mathbf{F}$ são escolhidas de modo que $V_{F}$ seja assintoticamente não singular.

$\mathrm{O}$ vetor de funções $\mathbf{F}$ é um estimador consistente de $\mathbf{F}\left(\pi_{\mathrm{g}}\right)$. Portanto, pode-se ajustar um modelo linear $\mathbf{E}\left\{\mathbf{F}\left(\mathbf{p}_{\mathrm{g}}\right)\right\}=\mathbf{F}\left(\pi_{\mathrm{g}}\right)=\mathrm{X} \beta$, onde $\mathrm{X}$ é uma matriz conhecida, de dimensão $(u \times$ t) e $\beta$ é um vetor de parâmetros desconhecidos, de dimensão ( $\mathrm{t} \times 1$ ). $\mathrm{O}$ método dos mínimos quadrados ponderados é aplicado para determinar um estimador BAN, $\mathbf{b}$, para $\beta$, através de

$$
\mathbf{b}=\left(\mathbf{X}^{\prime} \mathbf{V}_{\mathbf{F}}^{-1} \mathbf{X}\right)^{-1} \mathbf{X}^{\prime} \mathbf{V}_{\mathbf{F}}^{-1} \mathbf{F}
$$

Testes estatísticos para o ajuste do modelo e para hipóteses lineares envolvendo $\beta$, podem ser efetuados através, respectivamente, de

$$
\begin{gathered}
\mathrm{X}^{2}=\operatorname{SS}(\mathbf{E}\{\mathbf{F}\}=\mathbf{X} \beta)=\mathbf{F}^{\prime} \mathbf{V}_{\mathbf{F}}^{-1} \mathbf{F}-\mathbf{b}^{\prime}\left(\mathbf{X}^{\prime} \mathbf{V}_{\mathbf{F}}^{-1} \mathbf{X}\right) \mathbf{b} \\
\mathbf{e} \\
\mathrm{X}^{2}=\operatorname{SS}(\mathbf{C} \beta=0)=\mathbf{b}^{\prime} \mathbf{C}^{\prime}\left[\mathbf{C}\left(\mathbf{X}^{\prime} \mathbf{V}_{\mathbf{F}}^{-1} \mathbf{X}\right)^{-1} \mathbf{C}^{\prime}\right]^{-1} \mathbf{C b} \\
\mathrm{O} \text { método } \mathrm{KO} \text { assume que os dados são MCAR isto é, que a }
\end{gathered}
$$
probabilidade de resposta independe dos dados observados e dos dados não observados.

Koch et al. (1977) demonstraram que o método GSK pode ser utilizado para a análise de medidas repetidas categorizadas. Segundo os autores, em experimentos 
com medidas repetidas, cada individuo é observado em d diferentes condições e as respostas correspondentes são classificadas em termos de $L$ categorias. Assim, há $r=L^{d}$ possiveis perfis de respostas. Estes perfis de respostas podem ser indexados pelo vetor $\mathrm{j}$ $=\left(\mathrm{j}_{1}, \mathrm{j}_{2}, \ldots, \mathrm{j}_{\mathrm{d}}\right)$, onde $\mathrm{j}_{\mathrm{g}}=1,2, \ldots, \mathrm{L}$ para $\mathrm{g}=1,2, \ldots, \mathrm{d}$. Como resultado $\pi_{\mathrm{ij}}=\pi_{\mathrm{ij} 1, \mathrm{j} 2}$, jd representa a probabilidade conjunta do perfil de resposta $j$ para individuos selecionados aleatoriamente da i-ésima população. A partir dessas considerações, os autores formulam diversas hipóteses de interesse em experimentos com medidas repetidas categorizadas.

Woolson \& Clarke (1984), visando incluir dados incompletos na análise de medidas repetidas categorizadas, consideram uma não resposta como mais uma categoria de resposta e, a seguir, aplicam, de modo natural, o método GSK.

Seguindo o raciocínio dos autores, conside-se uma única variável resposta, que é discreta, com k-1 categorias de respostas. Além disso, assume-se que esta variável deva ser medida em $\mathrm{N}$ indivíduos, em $\mathrm{t}$ pontos distintos no tempo, $\mathrm{T}_{1}, \mathrm{~T}_{2}, \ldots$, $T_{1}$. Sendo assim, cada um dos $\mathrm{N}$ indivíduos tem um perfil de resposta que pode ser classificado em uma das $(\mathrm{k}-1)^{\mathrm{t}}$ categorias mutuamente exclusivas.

Frequentemente, com dados longitudinais, alguns dos $\mathrm{N}$ indivíduos podem não ter a variável resposta medida em um ou mais pontos do tempo. Em tal situação, é útil considerar uma k-ésima categoria para a variável resposta em cada ponto no tempo, onde esta k-ésima categoria representa uma resposta perdida naquele ponto do tempo. Assim, os dados podem ser imaginados como uma tabela de $\mathrm{k}^{\mathrm{l}}-1$, ao invés de ( $\mathrm{k}$ $1)^{t}$ categorias, onde há $k^{1}-1$ categorias, em vez de $k^{1}$, visto que uma das $k^{1}$ categorias representaria dados incompletos para todos os pontos no tempo.

Os autores aplicaram a metodologia precedente a um estudo de fator de risco em coronárias de crianças na idade escolar. Levantamentos de coorte ("crosssectional") foram conduzidos em escolas nos anos de 1977, 1979 e 1981. Em cada um dos três levantamentos, a participação das crianças foi classificada em obesa $(0)$ ou não (N). O objetivo era modelar a probabilidade de obesidade, em função da idade e do 
sexo. Nesse caso, há duas categorias de respostas em cada ponto no tempo (O e N), que em conjunto com a categoria representando a resposta perdida (M-"missing") formam as três possíveis respostas. No exemplo, há três pontos no tempo em que a resposta é avaliada, então há 26 categorias de resposta $\left(k=3, t=3\right.$ e $\left.3^{3}-1=26\right)$. Os autores concluiram que probabilidades preditas de obesidade podem ser obtidas, sem influência do sexo, por $\operatorname{Pr}($ obesidade $)=-0,2069+0,0684 \times$ idade $-0,0026 \times$ idade $^{2}$.

Ogliari (1984), visando estudar os problemas causados pela aproximação assintótica dos testes utilizados em tabelas de contingência, utilizou o método GSK para efetuar análise de modelos log-lineares e verificar a independência, em tabelas do tipo CxDxF, obtidas por meio de simulação, de dados de populações com tamanhos variados. $\mathrm{O}$ autor verificou que:

- com tamanhos "pequenos" de amostras ( $N=200, N=250$ e $N=300)$, os testes estatísticos mostraram-se rigorosos em não rejeitar a estrutura de independência para as tabelas de contingência. Porém, os modelos ajustados aos dados nem sempre corresponderam exatamente aos que estavam sendo analisados;

- para tamanhos maiores de amostras $(\mathrm{N}=1000$ e $\mathrm{N}=1500)$, os testes estatísticos comportam-se de modo excelente e os modelos, que explicavam as relações entre as diversas variáveis em estudo, ficaram bem ajustados.

Segundo Little \& Rubin (1987) o mecanismo que govema os dados incompletos pode ser classificado em uma das três categorias:

1. Incompletos por razões totalmente aleatórias (MCAR - "missing completely at random"). Os dados são MCAR se a probabilidade de resposta é independente dos dados observados e dos dados não observados;

2. Incompletos ao acaso (MAR - "missing at random"). Os dados são MAR se a probabilidade de resposta depende somente dos dados observados;

3. Incompletos não ignoráveis. Os dados são não ignoráveis se a probabilidade de resposta depende dos dados não observados. 
Formalmente, seja $y_{i}=\left[y_{i 1}, y_{i 2}, \ldots, y_{i p}\right]^{\prime}$ um vetor $\mathrm{p} \times 1$ das medidas repetidas para o i-ésimo indivíduo. Considere $y_{i}$ particionado em dois componentes, $y_{i}{ }^{0} \mathrm{e}$ $y_{i}{ }^{m}$, onde $y_{i}^{o}$ é o vetor de dados observados e $y_{i}{ }^{m}$ é o vetor de dados não observados. Seja $u_{i}=\left[u_{i 1}, u_{i 2}, \ldots, u_{i p}\right]$ ' um vetor de variáveis aleatórias indicadoras, que descreve se as variáveis respostas, $y_{i j j}$, são observadas ou não $(j=1, \ldots, p)$. Os dados são MCAR se $u_{i}$ é independente de $y_{i}$. Nesse caso, tem-se que a densidade condicional $\pi\left(u_{i} / y_{i}\right)=$ $\pi\left(u_{i} / y_{i}^{o}, y_{i}^{m}\right)=$ densidade marginal $\pi\left(u_{i}\right)$. Alternativamente, os dados são MAR se $u_{i}$ é independente de $y_{i}{ }^{m}$, mas dependente de $y_{i}^{0}$, de modo que $\pi\left(u_{i} / y_{i}^{0}, y_{i}{ }^{m}\right)=\pi\left(u_{i} / y_{i}{ }^{0}\right)$. Quando $u_{i}$ depende de $y_{i}^{m}$, tem-se, então, um caso de dados incompletos não ignoráveis. Os métodos de análise de dados incompletos, em geral, assumem que os dados são MCAR.

As consequências de dados MCAR são saudáveis do ponto de vista de viés, pois as principais abordagens de análise são válidas. A grande dificuldade consiste em implementar a análise com dados incompletos. Análises que incluem apenas os dados completos e descartam as unidades com dados incompletos fornecem inferências válidas, embora possam significar perda de eficiência. Evidentemente, quando a suposição de MCAR é falsa, estas análises podem causar resultados viesados (Laird, 1988).

Little (1988) propôs um teste da suposição de MCAR para dados multivariados, baseado na razão de verossimilhança. Os dados são divididos em diversas subpopulações de acordo com seus padrões de dados incompletos. O teste de MCAR é, então, efetuado comparando-se as médias das subpopulações. Se os dados incompletos são MCAR, então as subpopulações têm a mesma matriz de médias e a mesma matriz de covariâncias. Assim, se as médias estimadas das subpopulações são diferentes, então o autor sugere que os dados não são do tipo MCAR.

Eskridge \& Mumm (1992) definem confiabilidade como sendo a probabilidade de uma dada cultivar superar uma outra (testemunha) em uma ampla variedade de ambientes. Formalmente, confiabilidade pode ser expressa por 


$$
\operatorname{Pr}\left(Y_{c}-Y_{1}>0\right)
$$

onde $Y_{c}$ e $Y_{t}$ são as respostas da c-ésima cultivar e da testemunha t, respectivamente, e Pr indica probabilidade. Os autores utilizam dados de ensaios internacionais de milho para ilustrar como confiabilidade de uma cultivar pode ser usada para ajudar a tomar decisões na escolha de cultivares superiores, bem como demonstrar como confiabilidade é relacionada com diversas medidas de estabilidade de cultivares.

Segundo os autores, a utilidade da confiabilidade em identificar cultivares superiores é baseada em duas suposições. Primeiro, o melhorista está principalmente interessado em identificar cultivares que tenham uma boa chance de superarem a testemunha, em ambientes onde a testemunha é normalmente cultivada. A segunda suposição é que os ensaios são conduzidos em ambientes que são representativos da população de ambientes onde a testemunha é bem adaptada.

Park \& Davis (1993), baseados no teste de Little (1988), desenvolveram um teste do mecanismo de dados incompletos para medidas repetidas categorizadas. Eles sugerem estratificar a população de acordo com os padrões de dados incompletos, ajustando os modelos de interesse dentro de cada estrato e, então, testar homogeneidade das estimativas dos parâmetros através dos estratos.

Os autores aplicaram essa metodologia ao exemplo utilizado por Woolson \& Clarke (1984), que trata de modelar a probabilidade de obesidade em função da idade e do sexo, em crianças na idade escolar. Verificaram, divergindo ligeiramente daqueles autores, que probabilidades preditas de obesidade podem ser calculadas através de $\operatorname{Pr}($ obesidade $)=\hat{\beta}_{0}+0,0638 \times$ idade $-0,00243 \times$ idade $^{2}$,

onde $\hat{\beta}_{0}=-0,1448$ para meninas com dados incompletos e $\hat{\beta}_{0}=-0,1965$ para todos os outros individuos, isto é, meninos e meninas com dados completos.

Torna-se evidente, portanto, que a inclusão de dados incompletos na análise de experimentos com medidas repetidas categorizadas, deve ser feita com cautela e de forma criteriosa. 
Lipsitz et al. (1994) propuseram um método, denotado por LLH (as iniciais dos autores), de dois passos para analisar medidas repetidas com dados incompletos. No primeiro passo é utilizado o método da máxima verossimilhança, enquanto que no segundo é utilizado o método dos minimos quadrados ponderados.

De acordo com os autores, seja $\Omega=\{1, \ldots, t\}$ o conjunto de $t$ ocasiões quando as medidas são feitas em todos indivíduos. Indivíduos com valores de covariáveis idênticos são agrupados no estrato $\mathrm{i}, \mathrm{i}=1, \ldots$, I. Os valores das covariáveis para o i-ésimo estrato podem ser arranjados em uma matriz $X_{i}$, de dimensão ( $t \times p$ ), sendo $X_{i}=\left[x_{i 1}, \ldots, x_{i t}\right]^{\prime}$, onde $X_{i t}$ é um vetor de covariáveis medido no tempo $t$ (e inclui intercepto e efeitos do tempo). Quando as covariáveis variam no tempo, isto é, $\mathrm{x}_{\mathrm{is}} \neq \mathrm{x}_{\mathrm{it}}$ para $s \neq t$, elas variam da mesma maneira para todos os $n_{i}$ indivíduos no estrato i. $O$ resultado para o indivíduo $\mathrm{k}\left(\mathrm{k}=1, \ldots, \mathrm{n}_{\mathrm{j}}\right)$ no estrato $\mathrm{i}$, no tempo $\mathrm{t}, \mathrm{y}_{\mathrm{ikt}}$, é binário, isto é, $\mathrm{y}_{\mathrm{ikt}}$ é 1 se a resposta é sucesso e 0 se a resposta é falha, no tempo t. Então, o vetor resposta para o indivíduo $k$, no estrato $i$, é $y_{i k}=\left[y_{i k l}, \ldots, y_{i k t}\right]^{\prime}$. $O$ vetor $E\left[y_{i k}\right]=\theta_{i}=$ $\left[\theta_{i 1}, \ldots, \theta_{i \mathfrak{i t}}\right]^{\prime}$ contém as probabilidades marginais no estrato $i$, onde $\theta_{\mathrm{it}}=\operatorname{Pr}\left(\mathrm{y}_{\mathrm{ikt}}=1\right)$.

Em geral, algum vetor de função, $\mathbf{F}\left(\theta_{\mathrm{i}}\right)$, das probabilidades marginais, no estrato $i$, é linearmente relacionado com as covariáveis $X_{i}$ e com os parâmetros $\beta$, isto é,

$$
\mathbf{F}\left(\theta_{\mathrm{i}}\right)=\mathrm{X}_{\mathrm{i}} \beta \text {. }
$$

O processo de estimação de $\beta$ é efetuado em dois passos.

Passo 1. Obter uma estimativa consistente $\left(\hat{\theta}_{\mathrm{i}}\right)$ do vetor de probabilidades marginais e de sua matriz de covariâncias, $V\left(\hat{\theta}_{i}\right)$, no estrato $i$.

Passo 2. Fazer $\hat{F}_{\mathrm{i}}=\mathbf{F}\left(\hat{\theta}_{\mathrm{i}}\right)$, que é consistente para $\mathbf{F}\left(\theta_{\mathrm{i}}\right)$ e assintoticamente normal multivariada. Uma estimativa consistente de sua matriz de covariâncias, derivada pelo

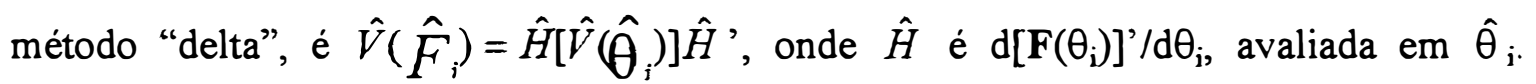
Então, para o modelo $\mathbf{F}\left(\theta_{\mathrm{i}}\right)=\mathrm{X}_{\mathrm{i}} \beta$, análise por mínimos quadrados ponderados é efetuada para consistentemente estimar $\beta$, usando os $\left\{\hat{F}_{i}\right\}$ como os vetores de respostas e as inversas dos $\left\{\hat{V}\left(\hat{F}_{i}\right)\right\}$ como as matrizes de ponderação: 


$$
\hat{\beta}=\left\{\sum_{i=1}^{J} \mathrm{X}_{\mathrm{i}}^{\prime}\left[\hat{V}\left(\hat{F}_{i}\right)\right]^{-1} \mathrm{X}_{\mathrm{i}}\right\}^{-1}\left\{\sum_{i=1}^{J} \mathrm{X}_{\mathrm{i}}^{\prime}\left[\hat{V}\left(\hat{F}_{i}\right)\right]^{-1} \hat{F}_{i}\right\}
$$

Uma estimativa consistente da matriz de covariâncias de $\hat{\beta}$ é

$$
\hat{V}(\hat{\beta})=\left\{\sum_{i=1}^{I} \mathrm{X}_{\mathrm{i}}^{\prime}\left[\hat{F}\left(\hat{F}_{i}\right)\right]^{-1} \mathrm{X}_{\mathrm{i}}\right\}^{-1}
$$

Segala (1997), utilizando três exemplos citados na literatura, comparou três métodos de análise de medidas repetidas categorizadas com dados incompletos: o método KO (Koch et al., 1972), o método WC (Woolson \& Clarke, 1984) e o método LLH (Lipsitz et al., 1994). O autor verificou que:

- quanto à facilidade computacional o método WC supera os demais;

- em todos os três exemplos analisados o método LLH forneceu os menores erros padrões das estimativas dos parâmetros, mas em dois deles proporcionou o pior ajuste do modelo.

Fonseca (1997) aplicou o teste do mecanismo de dados incompletos em medidas repetidas categorizadas, proposto por Park \& Davis (1993), utilizando o procedimento CATMOD do SAS (SAS, 1990), a um estudo de fator de risco em coronárias de crianças na idade escolar. Esse exemplo havia sido anteriormente estudado por Woolson \& Clarke (1984) e por Park \& Davis (1993).

Eskridge (1997) apresentou um método para avaliar e comparar híbridos de milho, que são candidatos a lançamento, baseado em suas probabilidades de superarem um outro híbrido (testemunha). Segundo o autor, o método é útil porque:

- é fácil de entender e pode ser implementado usando o procedimento CATMOD do SAS;

- usa toda informação disponível de dados incompletos, que surgem em ensaios de competição de cultivares;

- não faz suposições sobre a distribuição da característica de interesse;

- pode ser utilizado para comparar cultivares e avaliar os efeitos de variáveis ambientais sobre a probabilidade de cultivares superarem uma testemunha. 


\section{METODOLOGIA}

A metodologia, exposta a seguir, será aplicada em dois conjuntos de dados fictícios, ambos representando rendimentos de 3 cultivares de feijão (Phaseolus vulgaris, L), uma delas sendo a testemunha, provenientes de 72 ensaios distribuídos em 5 locais (regiões produtoras), em 3 anos (Tabelas 3 e 4). Os dados foram propositadamente escolhidos para mostrar algumas nuances do método de Eskridge (1997), que utiliza o conceito de confiabilidade para selecionar cultivares superiores, bem como do teste do mecanismo de dados incompletos (Park \& Davis, 1993), que verifica se os dados suportam a condição de MCAR. Além disso, é introduzido um modelo alternativo ao de Eskridge (1997) visando observar a utilidade desse modelo.

O interesse, nesse caso, é comparar a confiabilidade das duas cultivares, bem como testar se os dados são MCAR. 
Tabela 3. Dados de rendimentos de feijão ( $R 1, R 2, R 3)$, em $\mathrm{kg} / \mathrm{ha}$, de três cultivares ( $\mathrm{R} 3$ é o rendimento da testemunha), de acordo com o local e o ano do ensaio (Primeiro conjunto de dados).

\begin{tabular}{|c|c|c|c|c|c|c|c|c|c|}
\hline Local & Ano & $\mathrm{R} 1$ & $\mathrm{R} 2$ & $\mathrm{R} 3$ & Local & Ano & $\mathrm{R} 1$ & $\mathrm{R} 2$ & $\mathrm{R} 3$ \\
\hline$A$ & 88 & . & 1250 & 1280 & A & 88 & t. & 1400 & 1380 \\
\hline A & 88 & . & 1500 & 1450 & A & 88 & & 1380 & 1370 \\
\hline A & 88 & . & 1600 & 1580 & B & 88 & 1150 & & 1230 \\
\hline B & 88 & 1120 & 1125 & 1200 & B & 88 & 1118 & 1125 & 1210 \\
\hline B & 88 & 1225 & 1200 & 1330 & B & 88 & 1400 & 1280 & 1420 \\
\hline B & 88 & 1365 & 1300 & 1420 & B & 88 & 1210 & 1650 & 1400 \\
\hline B & 88 & 1425 & 1610 & 1475 & C & 88 & 1125 & 1710 & 1540 \\
\hline C & 88 & 1250 & 1310 & 1420 & B & 88 & 1520 & 1180 & 1270 \\
\hline B & 88 & 1380 & 1110 & 1250 & C & 88 & 1250 & 1270 & 1150 \\
\hline $\mathrm{M}$ & 88 & 1550 & 1487 & 1398 & A & 88 & 1480 & 1399 & 1298 \\
\hline A & 88 & 1575 & 1550 & 1530 & A & 88 & 1499 & 1255 & 1200 \\
\hline A & 88 & 1690 & 1550 & 1430 & B & 88 & 1290 & 1270 & 1221 \\
\hline $\mathbf{M}$ & 88 & 1300 & 1350 & 1125 & $N$ & 88 & 1310 & 1410 & 1198 \\
\hline $\mathbf{N}$ & 88 & 1390 & 1320 & 1421 & $\mathbf{M}$ & 88 & 1450 & 1490 & 1298 \\
\hline A & 88 & 1299 & 1250 & 1370 & $\mathrm{~N}$ & 88 & 1497 & 1420 & 1401 \\
\hline $\mathbf{M}$ & 88 & 1550 & 1490 & 1280 & $\mathbf{M}$ & 88 & 1290 & 1350 & 1186 \\
\hline C & 89 & 1299 & 1450 & 1311 & C & 89 & 1250 & 1418 & 1255 \\
\hline C & 89 & 1280 & 1411 & 1310 & C & 89 & 1200 & 1390 & 1280 \\
\hline A & 89 & . & 1450 & 1350 & $\mathbf{M}$ & 89 & . & 1498 & 1354 \\
\hline $\mathbf{M}$ & 89 & . & 1410 & 1325 & $\mathbf{M}$ & 89 & . & 1456 & 1222 \\
\hline $\mathbf{M}$ & 89 & 1290 & 1150 & 1423 & $\mathrm{M}$ & 89 & 1256 & 1326 & 1359 \\
\hline M & 89 & 1285 & 1299 & 1310 & A & 89 & 1380 & 1456 & 1390 \\
\hline M & 89 & 1200 & 1550 & 1389 & M & 89 & 1589 & 1250 & 1501 \\
\hline $\mathrm{M}$ & 89 & 1610 & 1290 & 1411 & $\mathrm{M}$ & 89 & 1565 & 1598 & 1480 \\
\hline$M$ & 89 & 1369 & 1454 & 1286 & $M$ & 89 & 1390 & & 1284 \\
\hline $\mathbf{M}$ & 90 & . & 1324 & 1413 & $M$ & 90 & . & 1365 & 1412 \\
\hline$M$ & 90 & . & 1290 & 1398 & M & 90 & . & 1510 & 1498 \\
\hline M & 90 & . & 1510 & 1390 & $N$ & 90 & 1366 & & 1399 \\
\hline$M$ & 90 & 1290 & . & 1325 & $\mathbf{M}$ & 90 & 1366 & 1391 & 1412 \\
\hline M & 90 & 1450 & 1270 & 1522 & M & 90 & 1360 & 1390 & 1380 \\
\hline M & 90 & 1310 & 1490 & 1390 & M & 90 & 1290 & 1499 & 1356 \\
\hline M & 90 & 1421 & . & 1395 & M & 90 & 1500 & 1390 & 1420 \\
\hline M & 90 & 1650 & 1385 & 1501 & $\mathbf{M}$ & 90 & 1601 & 1225 & 1425 \\
\hline$M$ & 90 & 1390 & 1410 & 1250 & M & 90 & 1450 & 1400 & 1289 \\
\hline $\mathrm{M}$ & 90 & 1550 & 1480 & 1320 & $\mathbf{M}$ & 90 & 1580 & 1450 & 1380 \\
\hline $\mathbf{M}$ & 90 & 1650 & 1510 & 1290 & $\mathrm{M}$ & 90 & 1520 & 1450 & 1250 \\
\hline
\end{tabular}


Tabela 4. Dados de rendimentos de feijão (R1, R2, R3), em kg/ha, de três cultivares (R3 é o rendimento da testemunha), de acordo com o local e o ano do ensaio (Segundo conjunto de dados).

\begin{tabular}{|c|c|c|c|c|c|c|c|c|c|}
\hline Local & Ano & R1 & R2 & R3 & Local & Ano & Rl & R2 & $\mathrm{R3}$ \\
\hline$A$ & 88 & . & 1290 & 1356 & $A$ & 88 & . & 1460 & 1380 \\
\hline A & 88 & . & 1389 & 1356 & A & 88 & . & 1492 & 1420 \\
\hline A & 88 & . & 1395 & 1356 & B & 88 & 1297 & . & 1365 \\
\hline B & 88 & 1298 & 1320 & 1360 & B & 88 & 1297 & 1320 & 1365 \\
\hline B & 88 & 1356 & 1330 & 1420 & B & 88 & 1365 & 1320 & 1392 \\
\hline B & 88 & 1361 & 1290 & 1489 & B & 88 & 1351 & 1478 & 1402 \\
\hline B & 88 & 1520 & 1295 & 1360 & C & 88 & 1652 & 1321 & 1456 \\
\hline C & 88 & 1265 & 1325 & 1425 & $B$ & 88 & 1698 & 1380 & 1456 \\
\hline B & 88 & 1690 & 1385 & 1450 & C & 88 & 1520 & 1621 & 1490 \\
\hline $\mathrm{M}$ & 88 & 1650 & 1630 & 1592 & A & 88 & 1620 & 1600 & 1523 \\
\hline A & 88 & 1594 & 1552 & 1497 & A & 88 & 1613 & 1596 & 1485 \\
\hline A & 88 & 1580 & 1546 & 1456 & B & 88 & 1489 & 1395 & 1290 \\
\hline $\mathrm{M}$ & 88 & 1460 & 1490 & 1365 & $N$ & 88 & 1465 & 1498 & 1310 \\
\hline$N$ & 88 & 1458 & 1390 & 1489 & $M$ & 88 & 1423 & 1540 & 1369 \\
\hline A & 88 & 1520 & 1420 & 1550 & $N$ & 88 & 1620 & 1580 & 1550 \\
\hline M & 88 & 1590 & 1550 & 1456 & $M$ & 88 & 1490 & 1520 & 1398 \\
\hline C & 89 & 1390 & 1587 & 1432 & C & 89 & 1456 & 1587 & 1520 \\
\hline C & 89 & 1547 & 1230 & 1356 & C & 89 & 1594 & 1356 & 1387 \\
\hline A & 89 & . & 1532 & 1498 & $M$ & 89 & . & 1456 & 1365 \\
\hline M & 89 & . & 1523 & 1398 & $\mathrm{M}$ & 89 & . & 1487 & 1369 \\
\hline $\mathrm{M}$ & 89 & 1458 & 1326 & 1542 & $\mathrm{M}$ & 89 & 1356 & 1369 & 1420 \\
\hline $\mathrm{M}$ & 89 & 1330 & 1450 & 1498 & A & 89 & 1498 & 1458 & 1469 \\
\hline $\mathrm{M}$ & 89 & 1520 & 1463 & 1495 & $M$ & 89 & 1565 & 1395 & 1468 \\
\hline M & 89 & 1623 & 1395 & 1458 & M & 89 & 1421 & 1498 & 1347 \\
\hline M & 89 & 1439 & 1524 & 1385 & M & 89 & 1450 & + & 1346 \\
\hline M & 90 & . & 1380 & 1420 & M & 90 & . & 1345 & 1385 \\
\hline $\mathrm{M}$ & 90 & . & 1375 & 1395 & $M$ & 90 & . & 1450 & 1325 \\
\hline $\mathrm{M}$ & 90 & . & 1480 & 1420 & $M$ & 90 & 1360 & . & 1420 \\
\hline M & 90 & 1380 & . & 1435 & M & 90 & 1320 & 1350 & 1420 \\
\hline $\mathrm{M}$ & 90 & 1410 & 1350 & 1450 & $\mathrm{M}$ & 90 & 1360 & 1550 & 1460 \\
\hline$M$ & 90 & 1475 & 1280 & 1355 & $M$ & 90 & 1580 & 1350 & 1420 \\
\hline$M$ & 90 & 1450 & . & 1420 & M & 90 & 1480 & 1290 & 1360 \\
\hline $\mathrm{M}$ & 90 & 1650 & 1460 & 1520 & $M$ & 90 & 1535 & 1325 & 1450 \\
\hline $\mathrm{M}$ & 90 & 1425 & 1450 & 1350 & $M$ & 90 & 1550 & 1525 & 1485 \\
\hline$M$ & 90 & 1485 & 1465 & 1380 & $M$ & 90 & 1520 & 1490 & 1420 \\
\hline $\mathrm{M}$ & 90 & 1490 & 1450 & 1365 & $M$ & 90 & 1500 & 1455 & 1420 \\
\hline
\end{tabular}




\subsection{O Procedimento CATMOD: PROC CATMOD (SAS, 1990)}

O CATMOD é um procedimento que efetua análises por mínimos quadrados ponderados de funções generalizadas de dados categorizados, além de análises por máxima verossimilhança em modelos log-lineares.

O PROC CATMOD admite diversas declarações. Aqui são usadas as seguintes: MODEL, CONTRAST, POPULATION, REPEATED e RESPONSE. Neste caso, as especificações do CATMOD são:

\section{PROC CATMOD;}

MODEL resposta $=$ efeitos $</$ opções $>$;

CONTRAST "nome" descrição < , ..., descrição >;

POPULATION variáveis;

REPEATED < descrição do fator, ..., descrição do fator $><$ opções $>$; onde descrição do fator $=$ nome do fator $<$ níveis $>$;

RESPONSE < função >;

\section{Declaração PROC CATMOD}

A declaração PROC CATMOD é obrigatória e inicia o Procedimento CATMOD.

\section{Declaração MODEL}

A declaração MODEL é obrigatória.

A resposta indica as variáveis dependentes que determinam as categorias de respostas (as colunas da tabela de contingência). Aqui ela é apresentada pelas variáveis dependentes unidas por asterisco.

Os efeitos especificam as fontes de variação do modelo. A palavra-chave _RESPONSE_ pode ser usada como um efeito. Assim, estes efeitos determinam o 
número de parâmetros do modelo. Se não for usada a declaração POPULATION , o CATMOD usa estes efeitos para determinar as populações (as linhas da tabela de contingência). Os efeitos também podem ser definidos especificando a matriz do delineamento entre parênteses. As linhas da matriz do delineamento são separadas por vírgula.

As opções usadas neste trabalho são descritas a seguir.

ADDCELL = número, que adiciona um número para a frequência de cada casela;

FREQ, que formece a tabela de frequência de populações $\mathrm{x}$ respostas;

PRED, fornece os valores observados e preditos das funções das respostas para cada população, seus erros-padrões e os resíduos;

\section{Declaração CONTRAST}

A declaração CONTRAST forma e testa funções lineares dos parâmetros do modelo. Cada conjunto de efeitos (separados por vírgula) especifica uma linha ou conjunto de linhas da matriz $C$ que o $C A T M O D$ usa para testar a hipótese $C \beta=0$. A declaração CONTRAST deve ser precedida da declaração MODEL. A palavra-chave ALL_PARMS é considerada como um efeito com o número de parâmetros igual ao número de colunas da matriz do delineamento. Isto é particularmente útil quando a matriz do delineamento é fornecida diretamente na declaração MODEL.

O nome do contraste é obrigatório e deve conter no máximo 24 caracteres.

\section{Declaração POPULATION}

A declaração POPULATION indica que populações devem ser formadas a partir do cruzamento das variáveis especificadas. 


\section{Declaração REPEATED}

A declaração REPEATED é usada para incorporar medidas repetidas no modelo. Pode ser usada quando há mais que uma variável dependente e a palavra-chave _RESPONSE_é usada no modelo. Se as variáveis dependentes correspondem a um ou mais fatores de medidas repetidas, deve-se usar a declaração REPEATED para definir _RESPONSE_em termos daqueles fatores.

O valor de níveis é o número de níveis do fator de medidas repetidas identificado por um dado nome do fator.

\section{Declaração RESPONSE}

A declaração RESPONSE especifica funções das probabilidades de resposta.

\subsection{Método de Eskridge (1997)}

\subsubsection{Descrição}

O método de Eskridge utiliza-se do conceito de confiabilidade e do método WC.

Confiabilidade de uma cultivar é estimada pela probabilidade condicional que a cultivar tem de superar a testemunha, dado que a cultivar esteja presente no ambiente. Esta probabilidade condicional é a razão entre a probabilidade de que a cultivar supere a testemunha, estando a cultivar presente no ambiente, e a probabilidade que a cultivar esteja presente no ambiente. Estas probabilidades formarão um vetor de confiabilidade, que pode ser expresso por

$$
f(\hat{p})=\exp (B \otimes I) \log _{e}((A \otimes I) \hat{p})
$$


onde I indica matriz identidade, $\otimes$ é o produto direto e, $\mathrm{A}$ e $\mathrm{B}$ são matrizes que são escolhidas de forma conveniente. Este vetor constituirá a declaração "Response" do procedimento CATMOD do SAS.

Para utilizar o método WC, o autor agiu da seguinte maneira: para um ambiente particular (uma combinação local-ano) e um par híbrido-testemunha, o desempenho do híbrido assume o valor 1 se o híbrido supera a testemunha; 0 se o híbrido não supera a testemunha e -1 se o hibrido está ausente naquele ambiente. Estes três resultados possiveis constituem uma variável resposta categorizada com três categorias (1,0 e -1). Esta variável é medida em t híbridos, que são comparados com uma única testemunha em n ambientes. Assim, cada ambiente tem um perfil de resposta com um total de $3^{\mathrm{t}}-1$ categorias mutuamente exclusivas.

Para esclarecer melhor estas idéias, suponha-se os dados apresentados na Tabela 5.

Tabela 5. Frequência de locais, para cada categoria de resposta, para duas cultivares de feijão, em três anos.

\begin{tabular}{lrrrrrrrrr}
\hline Ano & \multicolumn{1}{c}{ Resposta } & & & & Total \\
& $-1,0$ & $-1,1$ & $0,-1$ & 0,0 & 0,1 & $1,-1$ & 1,0 & 1,1 & \\
\hline 88 & 1 & 4 & 1 & 8 & 3 & 0 & 2 & 13 & 32 \\
89 & 0 & 4 & 0 & 3 & 6 & 1 & 2 & 2 & 18 \\
90 & 3 & 2 & 2 & 2 & 3 & 1 & 3 & 6 & 22 \\
\hline
\end{tabular}

O resultado da primeira casela (1) significa que em 1988, em um local, a cultivar 1 estava ausente e, ao mesmo tempo, a cultivar 2 não superou a testemunha. $O$ resultado da segunda casela (4) significa que em 1988, em quatro locais, a cultivar 1 estava ausente e, ao mesmo tempo, a cultivar 2 superou a testemunha, e assim por diante.

Em 1988, a probabilidade condicional de que a cultivar 1 supere a testemunha é $0,556=(0+2+13) /(1+8+3+0+2+13)$, onde o denominador 32 é cancelado. 


\subsubsection{Modelo}

O modelo adotado por Eskridge (1997) é o seguinte:

$$
y_{i j}=\mu+a_{i}+c_{j}+a c_{i j}+e_{i j}
$$

com $\mathrm{i}=1, \ldots, \mathrm{I} ; \mathrm{j}=1, \ldots, \mathrm{J}$;

onde:

$\mathrm{y}_{\mathrm{ij}}$ é o valor observado da confiabilidade no i-ésimo ano e na j-ésima cultivar;

$\mu$ é uma constante inerente a todas as observações;

$a_{i}$ é o efeito do i-ésimo ano;

$c_{j}$ é o efeito da j-ésima cultivar;

$\mathrm{ac}_{\mathrm{ij}}$ é o efeito da interação do i-ésimo ano com a j-ésima cultivar;

$e_{i j}$ é o erro associado à observação $y_{i j}$.

\subsubsection{Um programa}

Para se efetuar a análise, utilizando o PROC CATMOD, para o primeiro conjunto de dados, pode-se utilizar o seguinte programa, adaptado de Eskridge (1997).

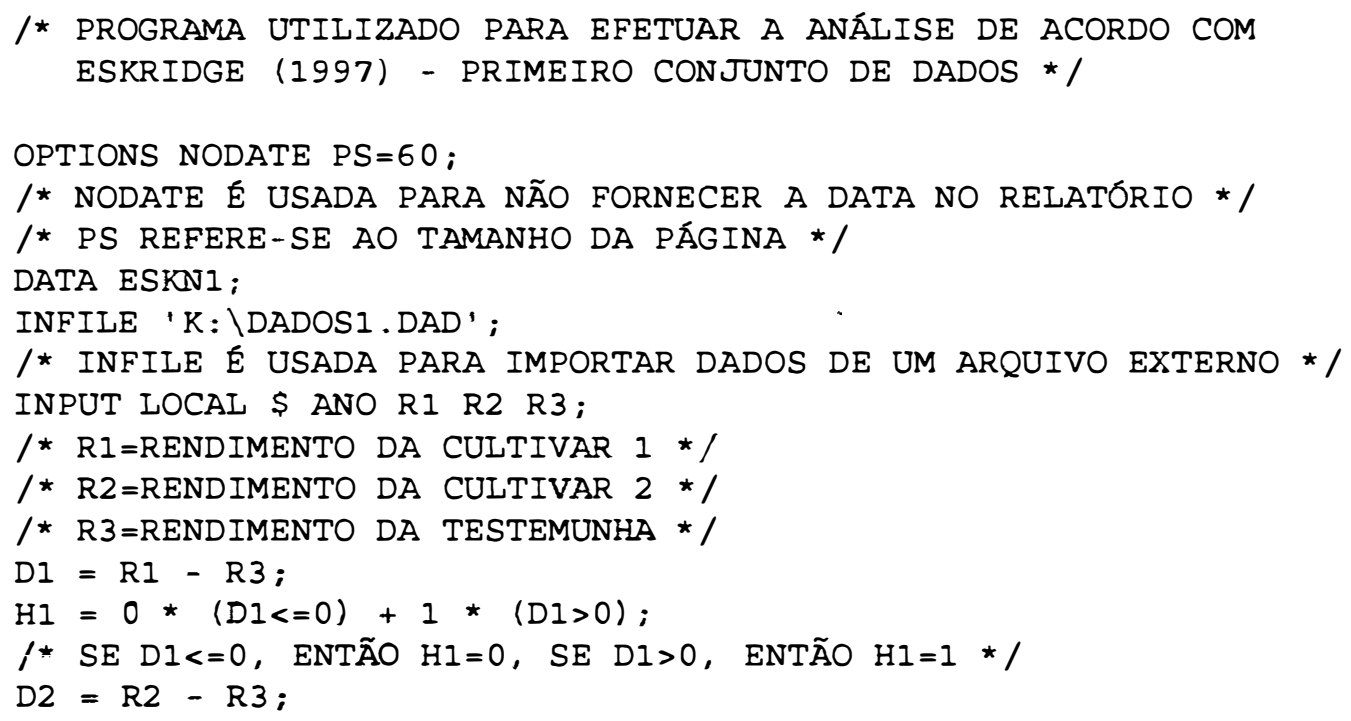




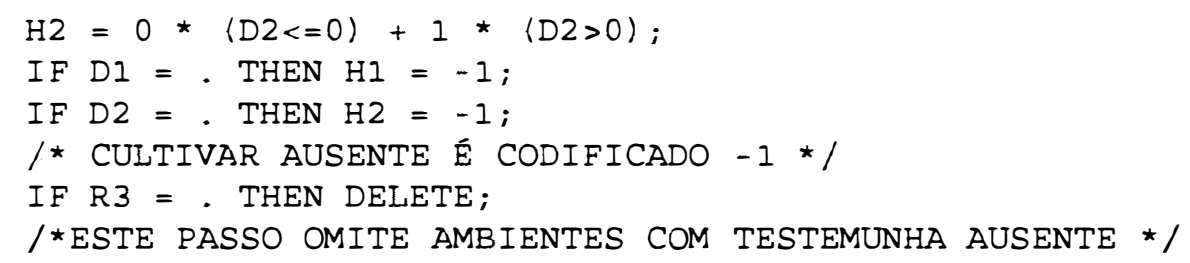

PROC CATMOD;

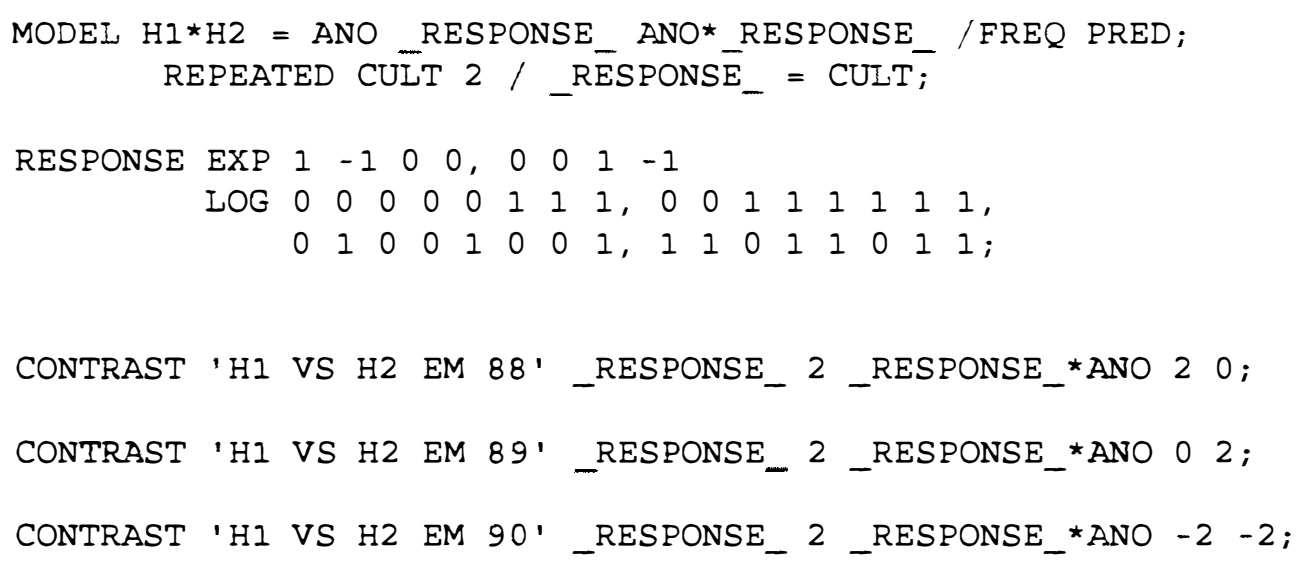

RUN ;

A declaração RESPONSE, neste caso, para cada população, especificará duas funções das probabilidades de resposta: a primeira representará a confiabilidade da cultivar 1 , enquanto a segunda representará a confiabilidade da cultivar 2 . Tome-se, por exemplo, a primeira população (ano de 88), cujo vetor de frequências observadas indicar-se-á por p. Os valores destas duas funções são obtidos da seguinte forma: sejam

$$
B=\left[\begin{array}{cccc}
1 & -1 & 0 & 0 \\
0 & 0 & 1 & -1
\end{array}\right], A=\left[\begin{array}{llllllll}
0 & 0 & 0 & 0 & 0 & 1 & 1 & 1 \\
0 & 0 & 1 & 1 & 1 & 1 & 1 & 1 \\
0 & 1 & 0 & 0 & 1 & 0 & 0 & 1 \\
1 & 1 & 0 & 1 & 1 & 0 & 1 & 1
\end{array}\right] \text { e } p=\left[\begin{array}{c}
1 \\
4 \\
1 \\
8 \\
3 \\
0 \\
2 \\
13
\end{array}\right]
$$


Então, calculando a expressão $\exp \left(\mathrm{B} \log _{\mathrm{e}}(\mathrm{A} \mathrm{p})\right.$, obtem-se o vetor das confiabilidades: $\left[\begin{array}{l}0,556 \\ 0,645\end{array}\right]$, onde 0,556 é o valor da confiabilidade da cultivar 1 em 88 e 0,645 é o valor da confiabilidade em 88 .

O primeiro contraste, CONTRAST 'H1 VS H2 EM 88'_RESPONSE_2_RESPONSE_*ANO 20 , significa comparar a confiabilidade da cultivar 1 com a confiabilidade da cultivar 2 , em 1988. Observa-se que, neste caso, os parâmetros envolvidos e seus coeficientes são: _RESPONSE_com coeficiente 2 e _RESPONSE_*ANO com coeficientes 20.

Os outros contrastes têm significados análogos ao primeiro.

Os parâmetros envolvidos no contraste e seus coeficientes serão melhor compreendidos no item seguinte.

Para a análise do segundo conjunto de dados basta alterar a declaração NFILE.

\subsubsection{Um programa alternativo}

Pode-se utilizar um programa alternativo, certamente mais compreensivel para pesquisadores pouco afeitos ao CATMOD, usando a matriz do delineamento diretamente na declaração MODEL. Neste caso, a matriz do delineamento é a seguinte:

$$
\left[\begin{array}{cccccc}
1 & 1 & 0 & 1 & 1 & 0 \\
1 & 1 & 0 & -1 & -1 & 0 \\
1 & 0 & 1 & 1 & 0 & 1 \\
1 & 0 & 1 & -1 & 0 & -1 \\
1 & -1 & -1 & 1 & -1 & -1 \\
1 & -1 & -1 & -1 & 1 & 1
\end{array}\right] .
$$


A matriz $\mathrm{X}$ tem, aqui, o mesmo papel que desempenha em regressão múltipla padrão, isto é, ela indica a maneira como a variação entre um certo conjunto de funções $F(\pi)$ das probabilidades das caselas pode ser caracterizado como funções lineares de um conjunto de parâmetros desconhecidos.

No caso em apreço, as colunas representam os parâmetros e as linhas as funções de interesse do pesquisador.

A primeira coluna representa o efeito do intercepto, a segunda e a terceira representam o efeito de anos, e assim por diante.

A primeira e a segunda linha representam, respectivamente, as confiabilidades das cultivares 1 e 2 , em 88 . A terceira e a quarta linha representam, respectivamente, as confiabilidades das cultivares 1 e 2 , em 89 . A quinta e a sexta linha representam, respectivamente, as confiabilidades das cultivares 1 e 2 , em 90 .

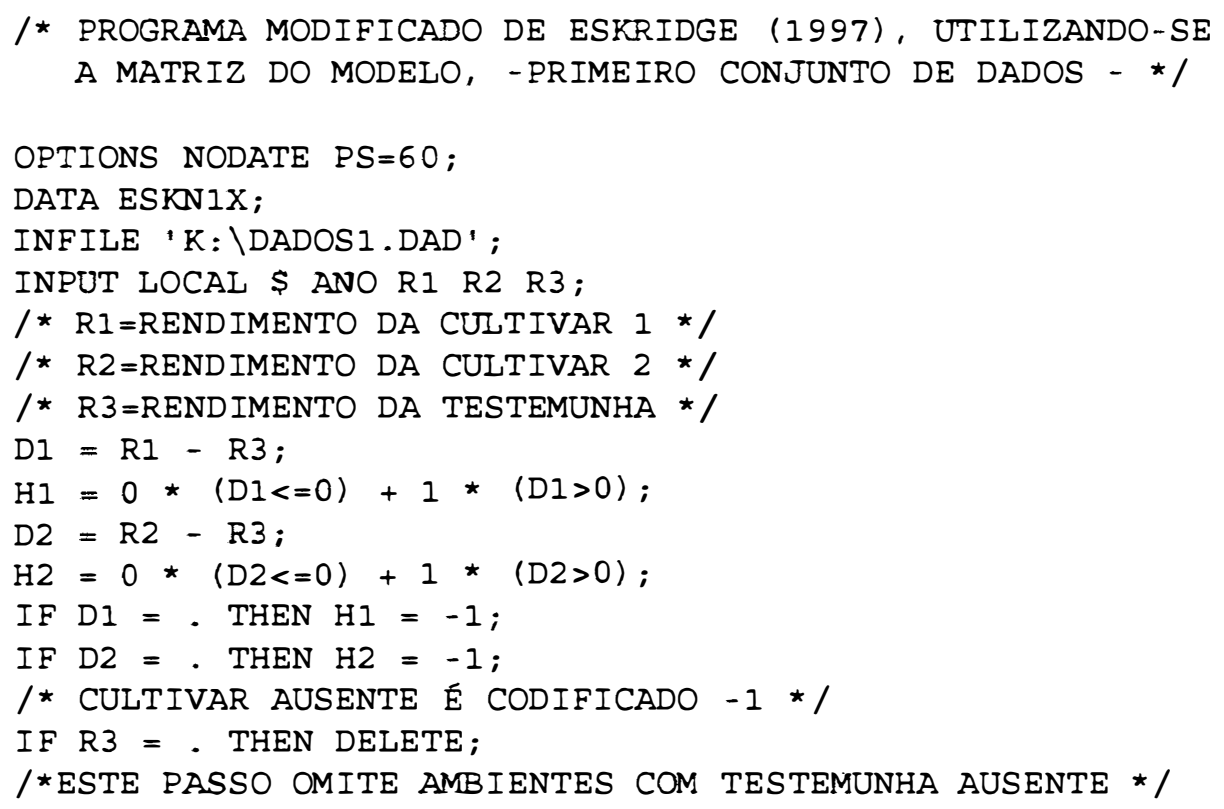


PROC CATMOD;

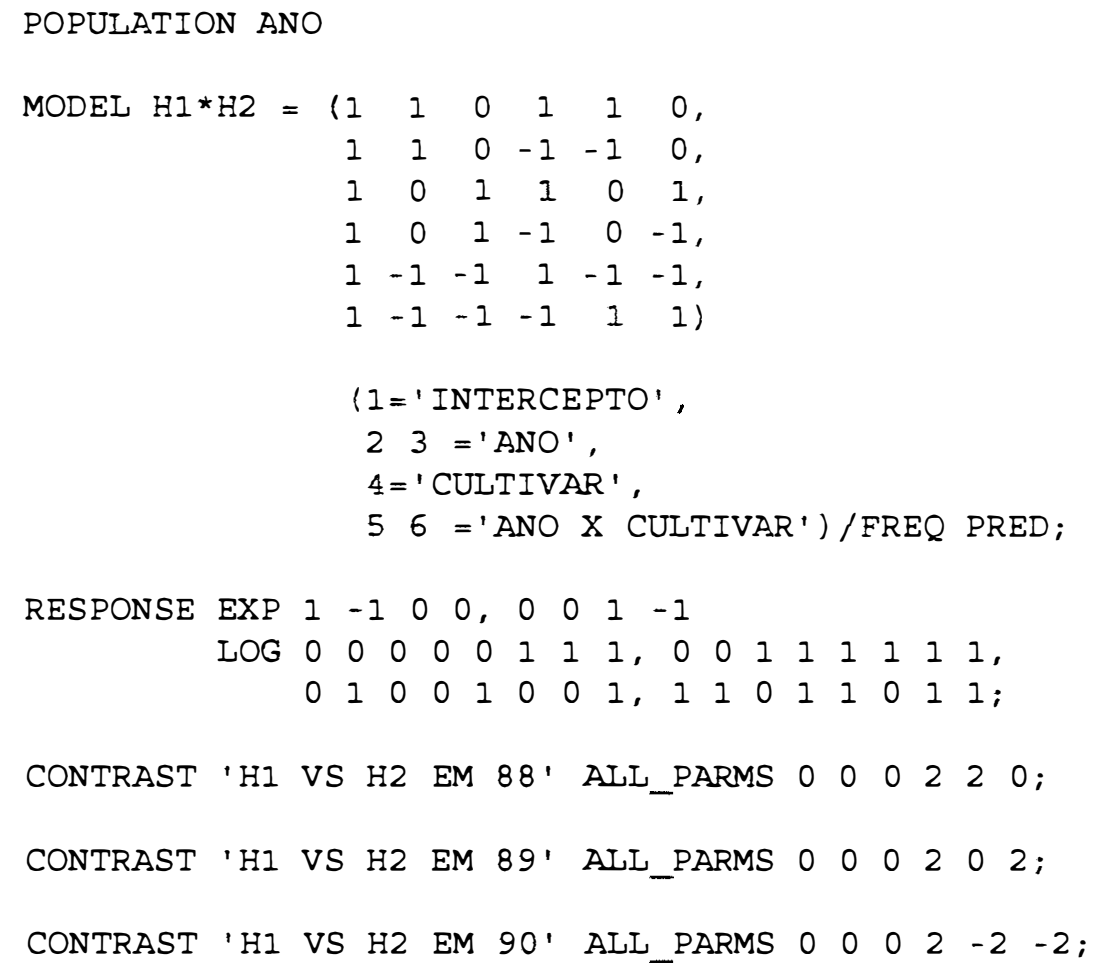

RUN ;

$$
\text { O primeiro contraste, }
$$

CONTRAST 'H1 VS H2 EM 88' ALL_PARMS 0000220 0,

significa comparar a confiabilidade da cultivar 1 com a confiabilidade da cultivar 2 , em 88. Os coeficientes deste contraste são obtidos da matriz $X$ da seguinte forma: subtrai-se os coeficientes da segunda linha dos seus correspondentes da primeira linha.

O significado dos outros contrastes e a obtenção de seus coeficientes são análogos ao primeiro.

Deve-se destacar que, conforme SAS (1990), quando a matriz do delineamento, que o próprio sistema fornece, mesmo quando não solicitado ("default"), é usada diretamente na declaração MODEL, o conteúdo da tabela da análise de variância pode variar em função de que se foram ou não fornecidos subconjuntos específicos de parâmetros a serem testados. Se forem fornecidos subconjuntos específicos, então a 
tabela contém um teste para cada subconjunto. Caso contrário, a tabela contém um teste para o que ele chama de efeito "MODEL / MEAN".

Como se verá, posteriormente, neste trabalho, este efeito "MODEL I MEAN", pode conduzir a resultados incoerentes na análise de variância, inclusive nos graus de liberdade.

Desta forma, para garantir que os resultados, referentes a cada fonte de variação, estão coerentes, foram incorporados ao programa anterior os seguintes contrastes:

CONTRAST 'INTERCEPTO' ALL_PARMS 100000 ;

CONTRAST 'ANO' ALL_PARMS 010000 , ALL_PARMS 001000 ;

CONTRAST 'CULTIVAR' ALL_PARMS 0000100 ; CONTRAST 'ANO X CULTIVAR' ALL_PARMS 000010 , ALL_PARMS 0000001 ;

Para a análise do segundo conjunto de dados basta alterar a declaração INFRE.

\subsection{Modelo alternativo}

\subsubsection{Justificativa}

O modelo utilizado por Eskridge (1997), no qual a variável ano é incluida, tem o inconveniente de que o pesquisador ou indica uma única cultivar para todos os locais ou não indica nenhuma. Com este modelo, se houver interação entre cultivares e locais, algum local certamente seria prejudicado, ocasionando perdas financeiras para os produtores deste local. Para evitar esta situação, pode-se suprimir ano e incluir local no modelo. 


\subsubsection{Modelo}

O modelo alternativo é o seguinte:

$$
y_{i j}=\mu+l_{i}-c_{j}+1 c_{i j}+e_{i j}
$$

$\operatorname{com} \mathrm{i}=1, \ldots, \mathrm{I} ; \mathrm{j}=1, \ldots, \mathrm{J}$;

onde:

$y_{\mathrm{ij}}$ é o valor observado da confiabilidade no i-ésimo local e na j-ésima cultivar;

$\mu$ é uma constante inerente a todas as observações;

$1_{\mathrm{i}}$ é o efeito do i-ésimo local;

$\mathrm{c}_{\mathrm{j}} \quad$ é o efeito da j-ésima cultivar;

$1 c_{i j}$ é o efeito da interação do i-ésimo local com a j-ésima cultivar;

$\mathrm{e}_{\mathrm{ij}}$ é o erro associado à observação $\mathrm{y}_{\mathrm{ij}}$.

\subsubsection{Um programa}

Para se efetuar a análise, utilizando o PROC CATMOD, para o primeiro conjunto de dados, pode-se utilizar o seguinte programa, adaptado de Eskridge (1997).

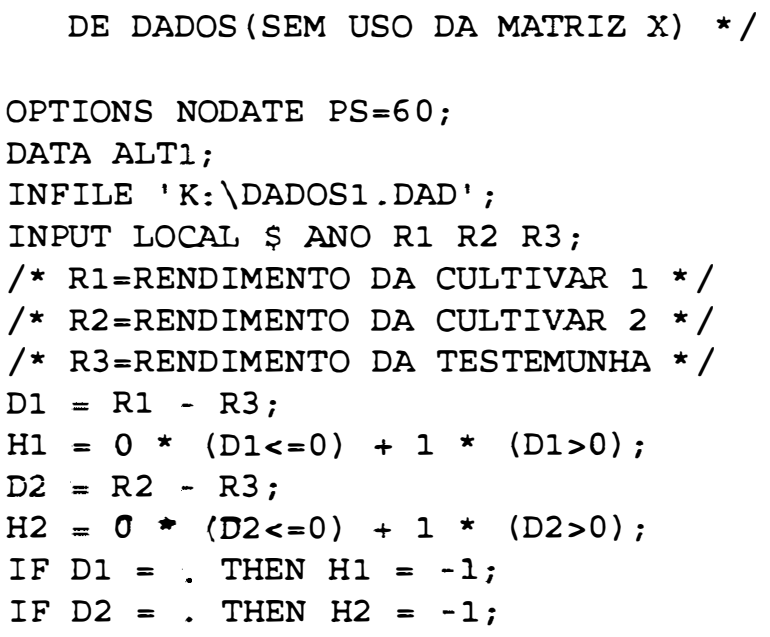


/* CULTIVAR AUSENTE E CODIFICADO - 1 * /

IF R3 = . THEN DELETE;

/*ESTE PASSO OMItE AMBIENTES COM testemunha AUSENTE */

PROC CATMOD;

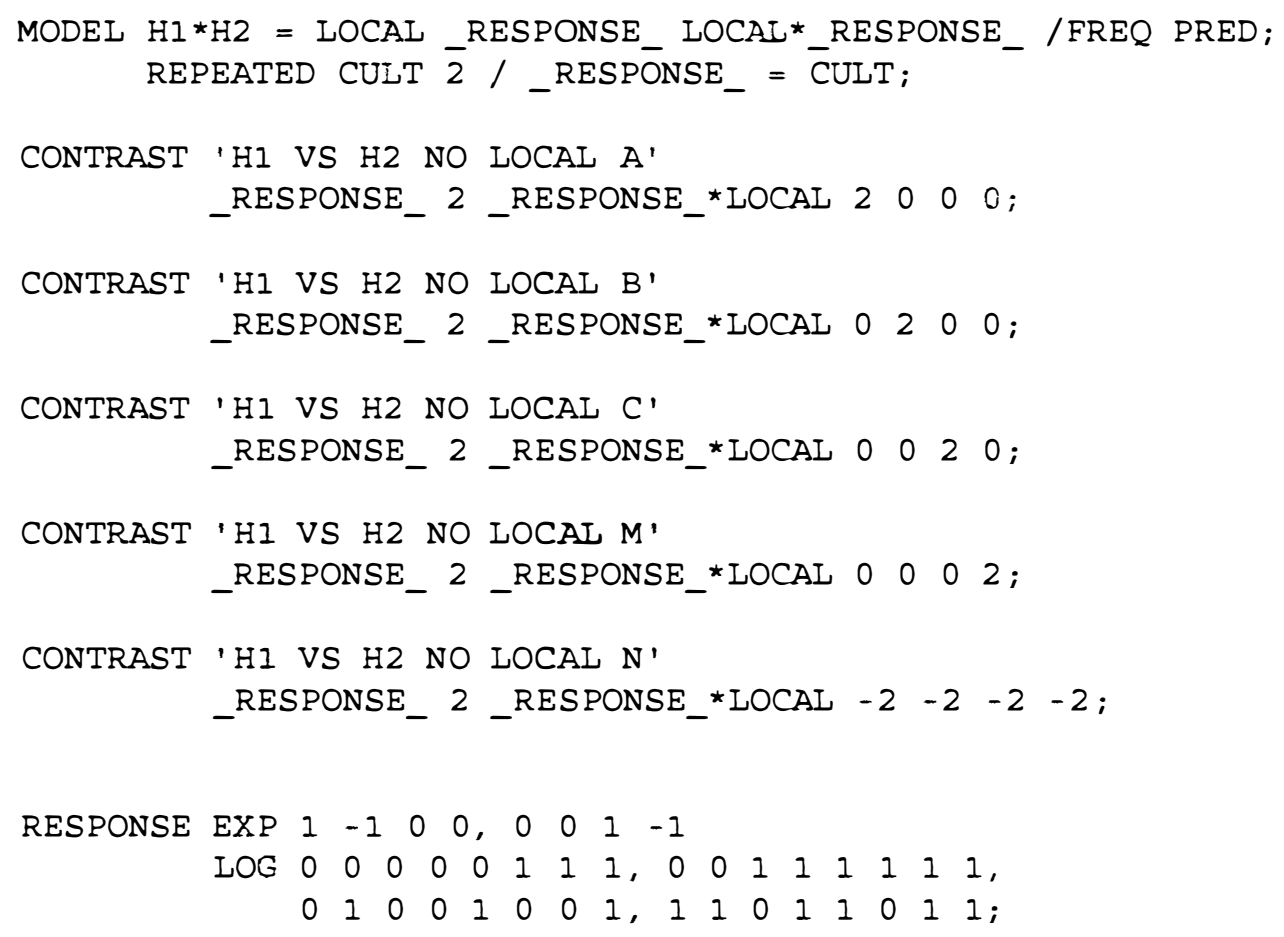

RUN ;

Para a análise do segundo conjunto de dados basta alterar a declaração INFILE.

\subsubsection{Um programa alternativo}

Pode-se, alternativamente, utilizar a matriz do delineamento diretamente como é mostrado no programa apresentado a seguir. Neste caso, a matriz do delineamento é 


$$
\left[\begin{array}{cccccccccc}
1 & 1 & 0 & 0 & 0 & 1 & 1 & 0 & 0 & 0 \\
1 & 1 & 0 & 0 & 0 & -1 & -1 & 0 & 0 & 0 \\
1 & 0 & 1 & 0 & 0 & 1 & 0 & 1 & 0 & 0 \\
1 & 0 & 1 & 0 & 0 & -1 & 0 & -1 & 0 & 0 \\
1 & 0 & 0 & 1 & 0 & 1 & 0 & 0 & 1 & 0 \\
1 & 0 & 0 & 1 & 0 & -1 & 0 & 0 & -1 & 0 \\
1 & 0 & 0 & 0 & 1 & 1 & 0 & 0 & 0 & 1 \\
1 & 0 & 0 & 0 & 1 & -1 & 0 & 0 & 0 & -1 \\
1 & -1 & -1 & -1 & -1 & 1 & -1 & -1 & -1 & -1 \\
1 & -1 & -1 & -1 & -1 & -1 & 1 & 1 & 1 & 1
\end{array}\right] .
$$

A matriz do delineamento tem a seguinte interpretação: cada coluna representa um parâmetro; cada linha representa a confiabilidade de uma das cultivares, em uma dada população (local).

A primeira coluna representa o intercepto; as quatro seguintes o efeito de locais, e assim por diante.

As duas primeiras linhas referem-se ao local A. A primeira representa a confiabilidade da cultivar 1 , enquanto a segunda representa a confiabilidade da cultivar 2.

As demais linhas têm sentido análogo.

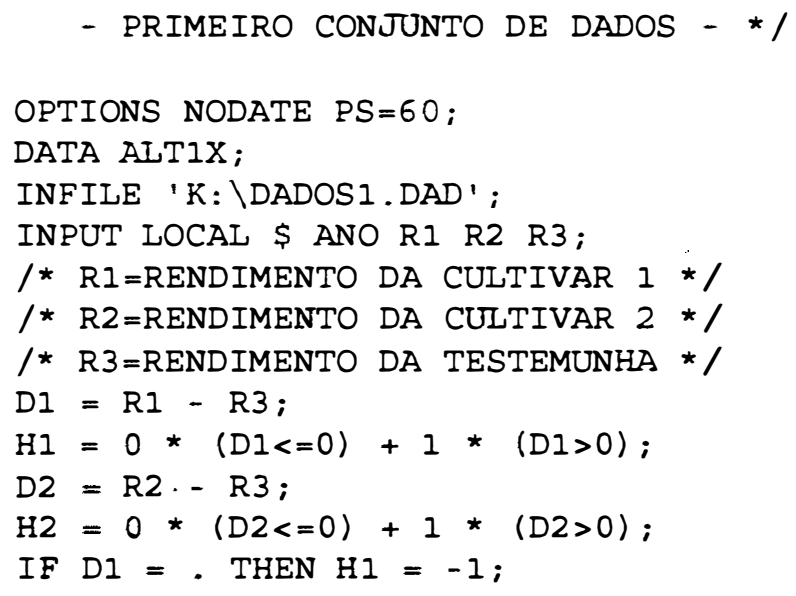




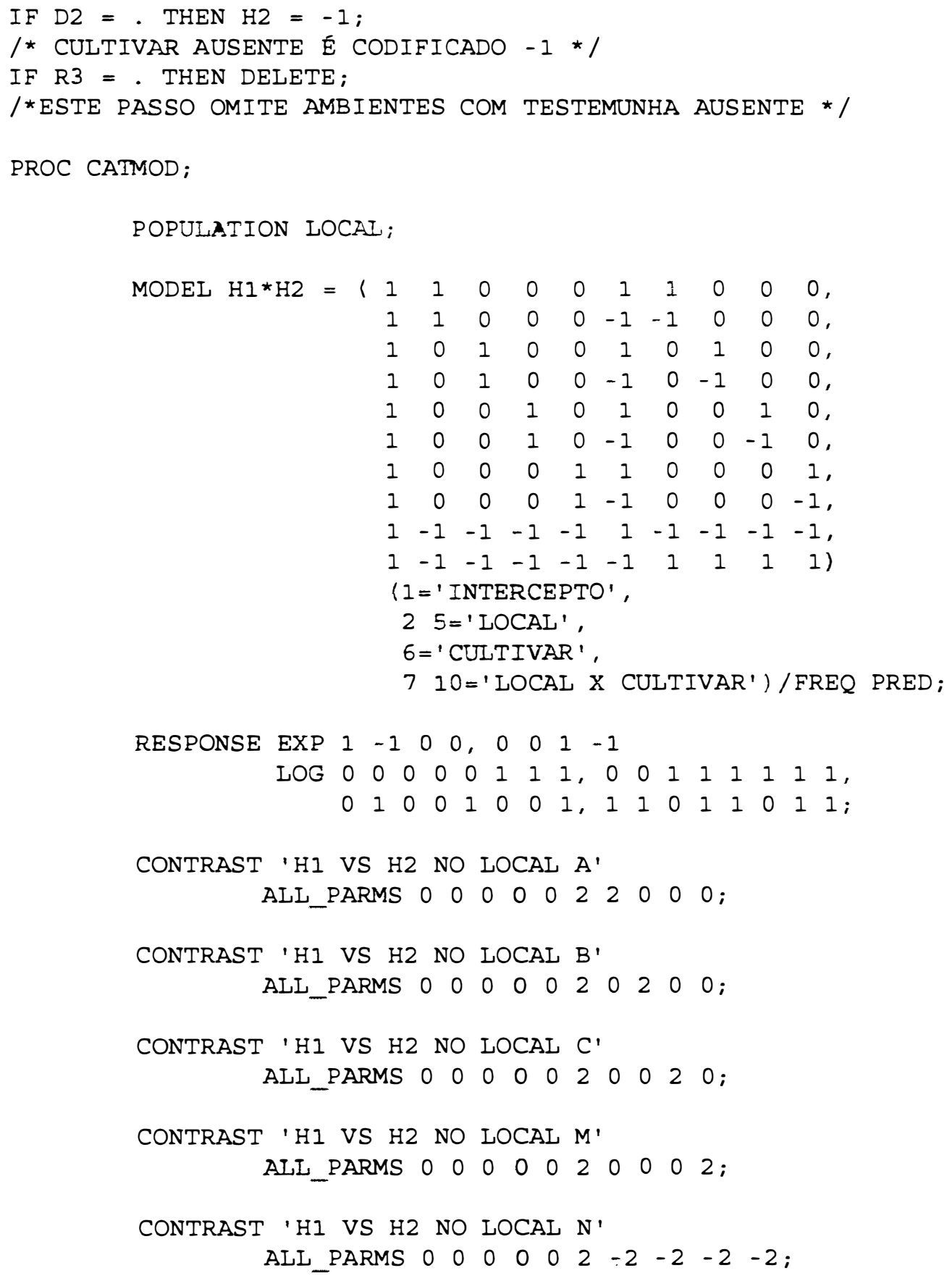


Para garantir resultados coerentes na análise de variância, de acordo com o exposto no item 3.2.4 (efeito "MODEL | MEAN"), foram incorporados ao programa anterior, os seguintes contrastes:

CONTRAST 'INTERCEPTO’ ALL_PARMS 100000000000 ;

CONTRAST 'LOCAL' ALL_PARMS 0100000000 ,

ALL_PARMS 0010000000 ,

ALL_PARMS 00001000000 ,

ALL_PARMS 0000100000 ,

ALL_PARMS 00000010000 ;

CONTRAST 'CULTIVAR' ALL_PARMS 00000010000 ;

CONTRAST 'LOCAL X CULTIVAR' ALL_PARMS 000000011000 ,

ALL_PARMS 0000000100 ,

ALL_PARMS 00000000010 ,

ALL_PARMS 00000000001 ;

Para a análise do segundo conjunto de dados basta alterar a declaração

INFILE.

3.4 Teste do mecanismo de dados incompletos (Park \& Davis, 1993)

\subsubsection{A estatística de Wald para homogeneidade de parâmetros específicos do estrato}

Seja $\mathrm{H}$ o número de estratos nos dados. Para o h-ésimo estrato, seja $\mathrm{n}_{\mathrm{h}} \mathrm{o}$ vetor das frequências observadas das caselas e $\pi_{\mathrm{h}} \mathrm{o}$ vetor das verdadeiras probabilidades. Seja $\mathrm{p}_{\mathrm{h}}$ o vetor correspondente de proporções amostrais com a matriz de covariâncias $\mathrm{V}_{\mathrm{h}}$. Seja $F\left(p_{h}\right)$ um vetor de $u_{h}$ funções de $p_{h}$. Então, os modelos de regressão de interesse são definidos como 


$$
E\left[F\left(p_{h}\right)\right]=X_{b} \beta_{h}, \text { para } h=1, \ldots, H,
$$

onde $X_{h}$ é uma matriz de dimensão $\left(u_{h} \times v\right)$, do modelo e $\beta_{h}$ é um vetor ( $\left.v x 1\right)$, de parâmetros desconhecidos. Definem-se os vetores compostos n e p como n' $=\left(n_{1}\right.$, . . , $\left.\mathrm{n}_{\mathrm{h}}{ }^{\prime}\right)$ e $\mathrm{p}^{\prime}=\left(\mathrm{p}_{1}{ }^{\prime}, \ldots, \mathrm{p}_{\mathrm{H}}{ }^{\prime}\right)$. Então, $\mathrm{V}=\operatorname{var}(\mathrm{p})=\operatorname{diag}\left(\mathrm{V}_{1}, \ldots, \mathrm{V}_{\mathrm{H}}\right)$. O modelo $(1)$ pode ser escrito como

$$
\mathrm{E}[\mathrm{F}(\mathrm{p})]=\mathrm{X} \beta \text {, }
$$

onde $\mathrm{X}=\operatorname{diag}\left(\mathrm{X}_{1}, \ldots, \mathrm{X}_{\mathrm{H}}\right)$ é uma matriz de dimensão $\left(\mathrm{u}^{*} \mathrm{x} \mathrm{v}^{*}\right)$ do modelo com $\mathrm{u}^{*}=$ $\sum_{h} u_{h}$ e $v^{*}=H v$, e $\beta=\left(\beta_{1}{ }^{\prime}, \ldots, \beta_{H}{ }^{\prime}\right)$.

A adequação do modelo (2) pode ser testada utilizando-se a estatística

$$
\mathrm{Q}=(\mathrm{F}-\mathrm{Xb})^{\prime} \mathrm{V}^{-1}(\mathrm{~F}-\mathrm{Xb}) \text {, }
$$

onde $b=\left(X^{\prime} V^{-1} X\right)^{-1} X^{\prime} V^{-1} F$ é o estimador de minimos quadrados ponderados de $\beta$. Um estimador consistente para a matriz de covariâncias de $\beta$ é dado por

$$
\mathrm{V}_{\mathrm{b}}=\left(\mathrm{X}^{\prime} \mathrm{V}^{-1} \mathrm{X}\right)^{-1} \text {. }
$$

A hipótese de homogeneidade é, então,

$$
\mathrm{H}_{0}: \beta_{1}, \ldots, \beta_{\mathrm{H}} \text {. }
$$

Equivalentemente, $\mathrm{H}_{0}$ pode ser expressa, com uma escolha conveniente de $C$, fazendo-se $C \beta=0$. A estatística de Wald para $\mathrm{H}_{0}$ é dada por

$$
\mathrm{Q}=(\mathrm{Cb})^{\prime}\left(\mathrm{C}\left(\mathrm{X}^{\prime} \mathrm{V}^{-1} \mathrm{X}\right)^{-1}(\mathrm{Cb})\right.
$$

que tem, aproximadamente, uma distribuição de qui-quadrado com $\mathrm{d}=\mathrm{v}(\mathrm{H}-1)$ graus de liberdade para grandes amostras, sob a hipótese de nulidade, onde $\mathrm{v}$ representa o número de parâmetros do modelo.

\subsubsection{A estatística de Wald para o mecanismo de dados incompletos}

Por conveniência, conforme sugestão dos autores, considera-se uma única variável resposta que tem c-1 categorias de respostas. Suponha-se que há $\mathrm{n}$ indivíduos e a variável resposta deva ser medida em cada um de $t$ pontos distintos no tempo, $T_{1}, \ldots$, $\mathrm{T}_{\mathbf{r}}$. Correspondentemente, cada um dos $\mathrm{n}$ indivíduos tem um perfil de resposta que pode 
ser classificado em uma das $(c-1)^{t}$ categorias mutuamente exclusivas. Frequentemente, alguns dos $\mathrm{n}$ indivíduos podem não ter a variável resposta medida em um ou mais pontos, no tempo. Nesta situação, é útil considerar-se a c-ésima categoria para a variável resposta em cada ponto no tempo, onde esta c-ésima categoria representa uma resposta perdida naquele ponto do tempo. Assim, os dados podem ser imaginados como uma tabela de $c^{t}-1$, ao invés de $(c-1)^{t}$ categorias, onde há $c^{t}-1$ categorias, ao invés de $c^{t}$, visto que uma das $c^{t}$ categorias representaria dados incompletos para todos os pontos no tempo.

Seja $\mathrm{H}$ o número de padrões distintos de dados incompletos. Desde que a categoria representando uma resposta incompleta é incluída como uma categoria adicional, o total $\mathrm{r}$ de categorias de respostas pode ser dividido em $\mathrm{H}$ grupos. Seja $\mathrm{S}_{\mathrm{h}} \mathrm{O}$ conjunto de categorias de respostas para o padrão de dados incompletos $h(h=1, \ldots, H)$ e seja $r_{h}$ o número de categorias de respostas em $S_{h}$. Por simplicidade, assume-se que $S_{1}$ indica o grupo de indivíduos que tem observações completas em todos os pontos no tempo.

Em vez de considerar-se $\mathrm{H}$ padrões de dados incompletos, consideram-se dois estratos $S_{1}$ e $S_{1}^{*}$, onde $S_{1}$ é o grupo de indivíduos que tem observações completas em todos os pontos no tempo e $S_{1}{ }^{*}$ é o grupo de indivíduos que tem pelo menos uma observação perdida. Assim,

$$
\mathrm{S}_{1}^{*}=\bigcup_{h=2}^{H} \mathrm{~S}_{\mathrm{h}} .
$$

Esta abordagem de dois estratos, segundo os autores, tem diversas vantagens: é útil para padrões específicos de dados incompletos, é fácil de aplicar e reduz o número de parâmetros a serem estimados. É esta abordagem de dois estratos que será utilizada neste trabalho.

Para verificar-se se os dados são MCAR, testa-se a homogeneidade dos parâmetros entre os casos com dados completos e com dados incompletos. Se há homogeneidade de parâmetros os dados são MCAR. 
ser classificado em uma das $(c-1)^{1}$ categorias mutuamente exclusivas. Frequentemente, alguns dos $\mathrm{n}$ indivíduos podem não ter a variável resposta medida em um ou mais pontos, no tempo. Nesta situação, é útil considerar-se a c-ésima categoria para a variável resposta em cada ponto no tempo, onde esta c-ésima categoria representa uma resposta perdida naquele ponto do tempo. Assim, os dados podem ser imaginados como uma tabela de $c^{t}-1$, ao invés de $(c-1)^{t}$ categorias, onde há $c^{t}-1$ categorias, ao invés de $c^{t}$, visto que uma das $c^{t}$ categorias representaria dados incompletos para todos os pontos no tempo.

Seja $\mathrm{H}$ o número de padrões distintos de dados incompletos. Desde que a categoria representando uma resposta incompleta é incluida como uma categoria adicional, o total $\mathrm{r}$ de categorias de respostas pode ser dividido em $\mathrm{H}$ grupos. Seja $\mathrm{S}_{\mathrm{h}} \mathrm{O}$ conjunto de categorias de respostas para o padrão de dados incompletos $h(h=1, \ldots, H)$ e seja $\mathrm{r}_{\mathrm{h}}$ o número de categorias de respostas em $\mathrm{S}_{\mathrm{h}}$. Por simplicidade, assume-se que $\mathrm{S}_{1}$ indica o grupo de indivíduos que tem observações completas em todos os pontos no tempo.

Em vez de considerar-se $\mathbf{H}$ padrões de dados incompletos, consideram-se dois estratos $S_{1}$ e $S_{1}^{*}$, onde $S_{1}$ é o grupo de indivíduos que tem observações completas em todos os pontos no tempo e $S_{1}{ }^{*}$ é o grupo de individuos que tem pelo menos uma observação perdida. Assim,

$$
\mathrm{S}_{1}^{*}=\bigcup_{h=2}^{H} \mathrm{~S}_{\mathrm{h}} .
$$

Esta abordagem de dois estratos, segundo os autores, tem diversas vantagens: é útil para padrões específicos de dados incompletos, é fácil de aplicar e reduz o número de parâmetros a serem estimados. É esta abordagem de dois estratos que será utilizada neste trabalho.

Para verificar-se se os dados são MCAR, testa-se a homogeneidade dos parâmetros entre os casos com dados completos e com dados incompletos. Se há homogeneidade de parâmetros os dados são MCAR. 


\subsubsection{Teste do mecanismo de dados incompletos usando anos no modelo}

A única maneira de se efetuar o teste do mecanismo de dados incompletos é fornecendo a matriz do delineamento diretamente na declaração MODEL. No caso presente, a matriz do delineamento é a seguinte:

$$
\left[\begin{array}{cccccccccccc}
1 & 1 & 0 & 1 & 1 & 0 & 0 & 0 & 0 & 0 & 0 & 0 \\
1 & 1 & 0 & -1 & -1 & 0 & 0 & 0 & 0 & 0 & 0 & 0 \\
0 & 0 & 0 & 0 & 0 & 0 & 1 & 1 & 0 & 1 & 1 & 0 \\
0 & 0 & 0 & 0 & 0 & 0 & 1 & 1 & 0 & -1 & -1 & 0 \\
1 & 0 & 1 & 1 & 0 & 1 & 0 & 0 & 0 & 0 & 0 & 0 \\
1 & 0 & 1 & -1 & 0 & -1 & 0 & 0 & 0 & 0 & 0 & 0 \\
0 & 0 & 0 & 0 & 0 & 0 & 1 & 0 & 1 & 1 & 0 & 1 \\
0 & 0 & 0 & 0 & 0 & 0 & 1 & 0 & 1 & -1 & 0 & -1 \\
1 & -1 & -1 & 1 & -1 & -1 & 0 & 0 & 0 & 0 & 0 & 0 \\
1 & -1 & -1 & -1 & 1 & 1 & 0 & 0 & 0 & 0 & 0 & 0 \\
0 & 0 & 0 & 0 & 0 & 0 & 1 & -1 & -1 & 1 & -1 & -1 \\
0 & 0 & 0 & 0 & 0 & 0 & 1 & -1 & -1 & -1 & 1 & 1
\end{array}\right] .
$$

A matriz do delineamento tem a seguinte interpretação: cada coluna representa um parâmetro; cada linha representa a confiabilidade de uma das cultivares, em uma dada população (ano), em um dado estrato (dados completos ou dados incompletos).

As seis primeiras colunas representam parâmetros dos dados completos, enquanto as seis últimas representam parâmetros dos dados incompletos. Entre as seis primeiras, a primeira representa o intercepto, a segunda e a terceira o efeito de anos, a quarta o efeito de cultivares, a quinta e a sexta o efeito da interação. As seis restantes têm sentido análogo.

As quatro primeiras linhas referem-se ao ano de 88 , as quatro seguintes referem-se ao ano de 89 e as quatro restantes referem-se ao ano de 90. 
A primeira linha representa a confiabilidade da cultivar 1 , com dados completos. A segunda linha representa a confiabilidade da cultivar 2 , com dados completos. A terceira linha representa a confiabilidade da cultivar 1 , com dados incompletos. A quarta linha representa a confiabilidade da cultivar 2 , com dados incompletos. As demais linhas têm sentido análogo.

Para testar o mecanismo de dados incompletos para o primeiro conjunto de dados, usando anos no modelo (Eskridge, 1997), através do CATMOD, foi elaborado o programa apresentado a seguir.

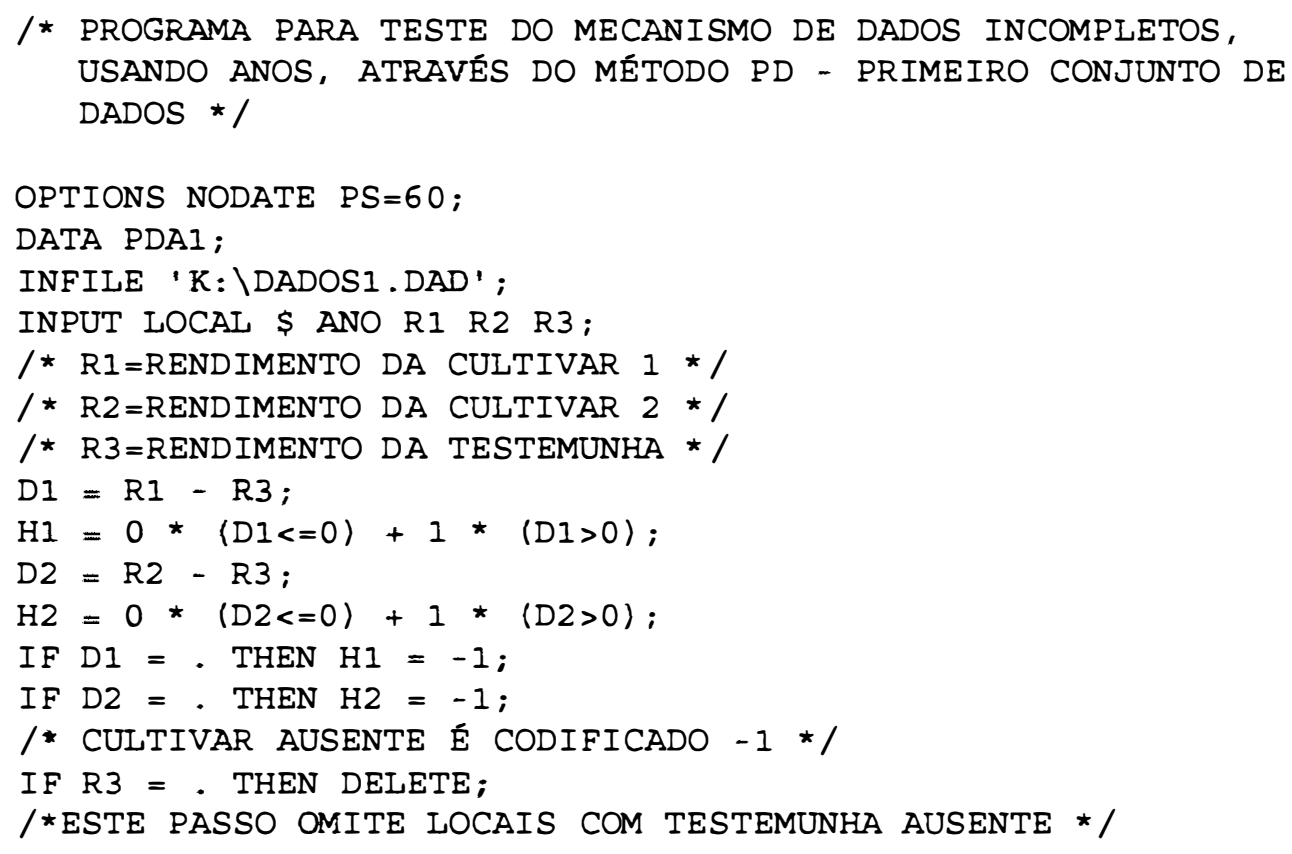


PROC CATMOD;

POPULATION ANO;

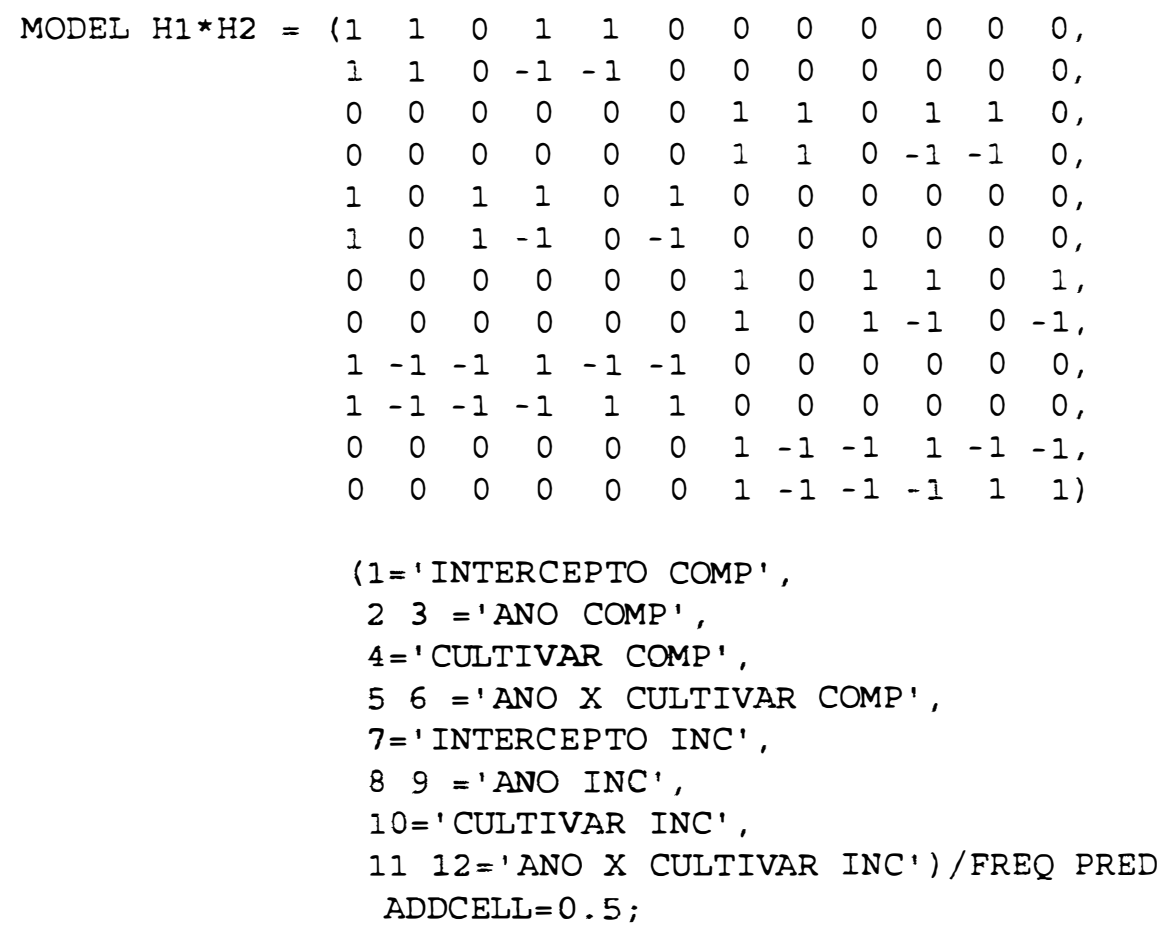

RUN ; 


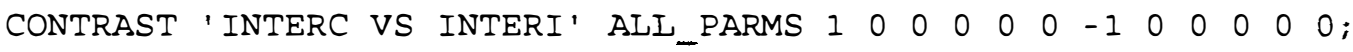
RUN ;



RUN ;

CONTRAST 'CULTC VS CULTI' ALI_PARMS $00 \begin{array}{lllllllllll}0 & 0 & 1 & 0 & 0 & 0 & 0 & 0 & -1 & 0 & 0\end{array}$ RUN ;

CONTRAST 'ANOCULTC VS ANOCULTI' ALI_PARMS $\begin{array}{llllllllllllllll}0 & 0 & 0 & 1 & 1 & 0 & 0 & 0 & 0 & -1 & -1 ;\end{array}$ RUN ;

A declaração

CONTRAST 'BETAC VS BETAI'

$$
\begin{array}{lllllllllllll}
\text { ALI_PARMS } & 1 & 0 & 0 & 0 & 0 & 0 & -1 & 0 & 0 & 0 & 0 & 0, \\
\text { ALI_PARMS } & 0 & 1 & 1 & 0 & 0 & 0 & 0 & -1 & -1 & 0 & 0 & 0, \\
\text { ALI_PARMS } & 0 & 0 & 0 & 1 & 0 & 0 & 0 & 0 & 0 & -1 & 0 & 0, \\
\text { ALI_PARMS } & 0 & 0 & 0 & 0 & 1 & 1 & 0 & 0 & 0 & 0 & -1 & -1 \text {; }
\end{array}
$$

é utilizada para comparar os parâmetros dos dados completos (BETAC) com os parâmetros dos dados incompletos (BETAI). Caso este contraste não seja significativo, então, segundo o teste de Park \& Davis (1993), os dados suportam a condição de MCAR.

Os demais contrastes servem para verificar que parâmetros especificos são diferentes, se for o caso.

A opção ADDCELL = 0.5 foi aplicada, neste e no programa seguinte, em virtude da ocorrência de matrizes de covariâncias singulares em algumas populações.

Para o segundo conjunto de dados basta alterar a declaração RNFILE. 


\subsubsection{Teste do mecanismo de dados incompletos usando locais no modelo}

Para testar o mecanismo dos dados incompletos para o primeiro conjunto de dados, usando locais no modelo (modelo alternativo), através do CATMOD, foi elaborado o seguinte programa:

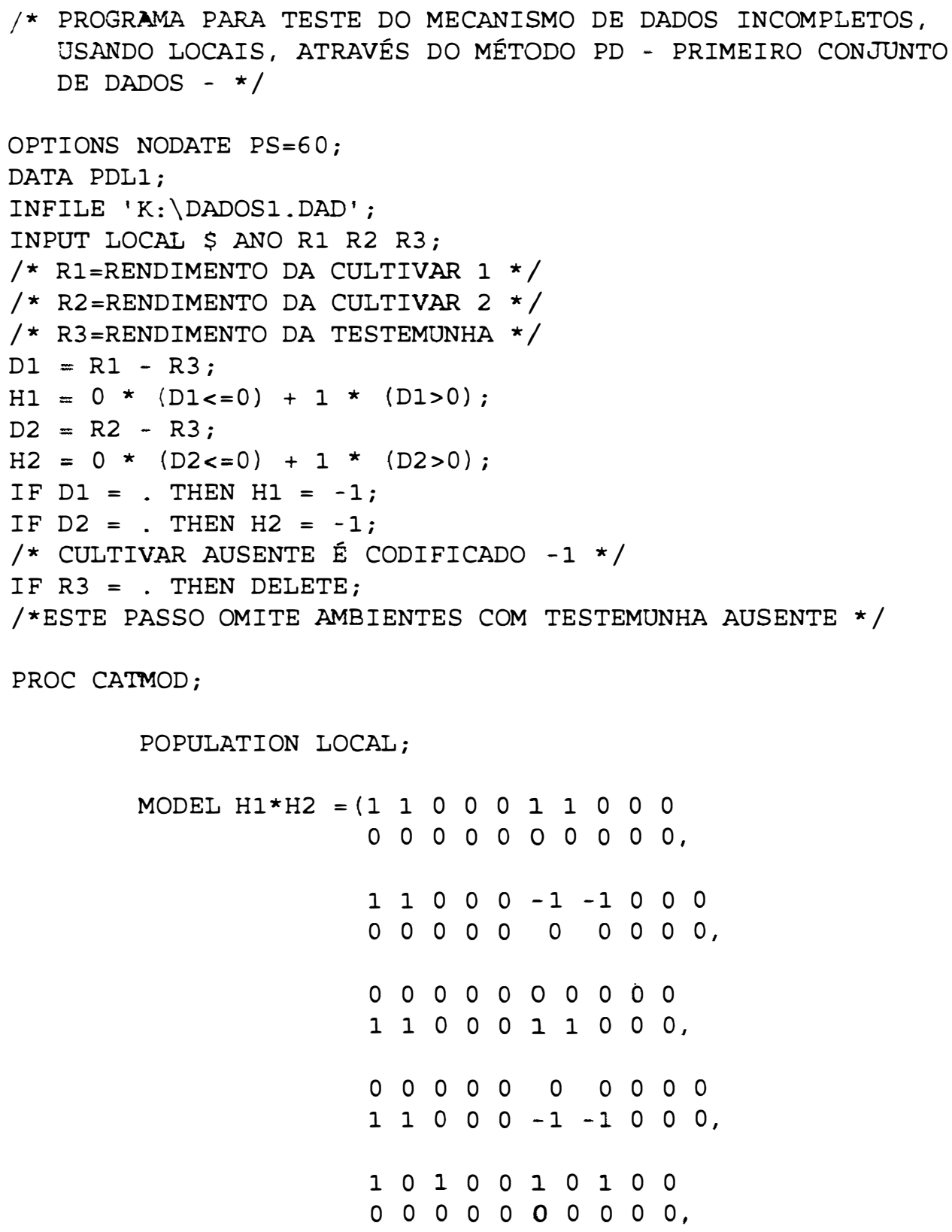




$$
\begin{aligned}
& \begin{array}{llllllllll}
1 & 0 & 1 & 0 & 0 & -1 & 0 & -1 & 0 & 0
\end{array}
\end{aligned}
$$

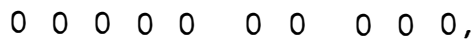



$$
\begin{aligned}
& \begin{array}{llllllllll}
1 & 0 & 1 & 0 & 0 & 1 & 0 & 1 & 0 & 0
\end{array} \text {, } \\
& \begin{array}{llllllllll}
0 & 0 & 0 & 0 & 0 & 0 & 0 & 0 & 0 & 0
\end{array} \\
& \begin{array}{lllllllllll}
1 & 0 & 1 & 0 & 0 & -1 & 0 & -1 & 0 & 0 & 0
\end{array}
\end{aligned}
$$



$$
\begin{aligned}
& 0000000000000 \text {, } \\
& \begin{array}{llllllllll}
1 & 0 & 0 & 1 & 0 & -1 & 0 & 0 & -1 & 0
\end{array} \\
& \begin{array}{llllllllll}
0 & 0 & 0 & 0 & 0 & 0 & 0 & 0 & 0 & 0
\end{array} \\
& 0 \begin{array}{llllllllll}
0 & 0 & 0 & 0 & 0 & 0 & 0 & 0 & 0 & 0
\end{array} \\
& \begin{array}{llllllllll}
1 & 0 & 0 & 1 & 0 & 1 & 0 & 0 & 1 & 0,
\end{array} \\
& \begin{array}{llllllllll}
0 & 0 & 0 & 0 & 0 & 0 & 0 & 0 & 0 & 0
\end{array}
\end{aligned}
$$

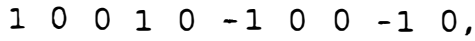

$$
\begin{aligned}
& \begin{array}{llllllllll}
1 & 0 & 0 & 0 & 1 & 1 & 0 & 0 & 0 & 1
\end{array} \\
& \begin{array}{llllllllll}
0 & 0 & 0 & 0 & 0 & 0 & 0 & 0 & 0 & 0
\end{array} \text {, } \\
& \begin{array}{llllllllll}
1 & 0 & 0 & 0 & 1 & -1 & 0 & 0 & 0 & -1
\end{array}
\end{aligned}
$$

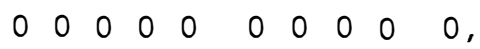

$$
\begin{aligned}
& 0 \begin{array}{llllllllll}
0 & 0 & 0 & 0 & 0 & 0 & 0 & 0 & 0 & 0
\end{array}
\end{aligned}
$$



$$
\begin{aligned}
& \begin{array}{llllllllll}
0 & 0 & 0 & 0 & 0 & 0 & 0 & 0 & 0 & 0
\end{array}
\end{aligned}
$$

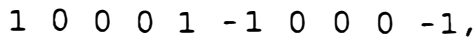

$$
\begin{aligned}
& \begin{array}{llllllllll}
1 & -1 & -1 & -1 & -1 & 1 & -1 & -1 & -1 & -1
\end{array}
\end{aligned}
$$

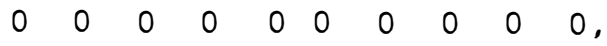

$$
\begin{aligned}
& \begin{array}{rrrrrrrrrr}
1 & -1 & -1 & -1 & -1 & -1 & 1 & 1 & 1 & 1 \\
0 & 0 & 0 & 0 & 0 & 0 & 0 & 0 & 0 & 0
\end{array} \\
& \begin{array}{llllllllll}
0 & 0 & 0 & 0 & 0 & 0 & 0 & 0 & 0 & 0
\end{array}
\end{aligned}
$$

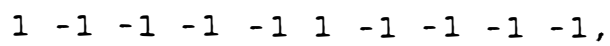

$$
\begin{aligned}
& \begin{array}{llllllllll}
0 & 0 & 0 & 0 & 0 & 0 & 0 & 0 & 0 & 0
\end{array} \\
& \begin{array}{lllllllllll}
1 & -1 & -1 & -1 & -1 & -1 & 1 & 1 & 1 & 1)
\end{array}
\end{aligned}
$$




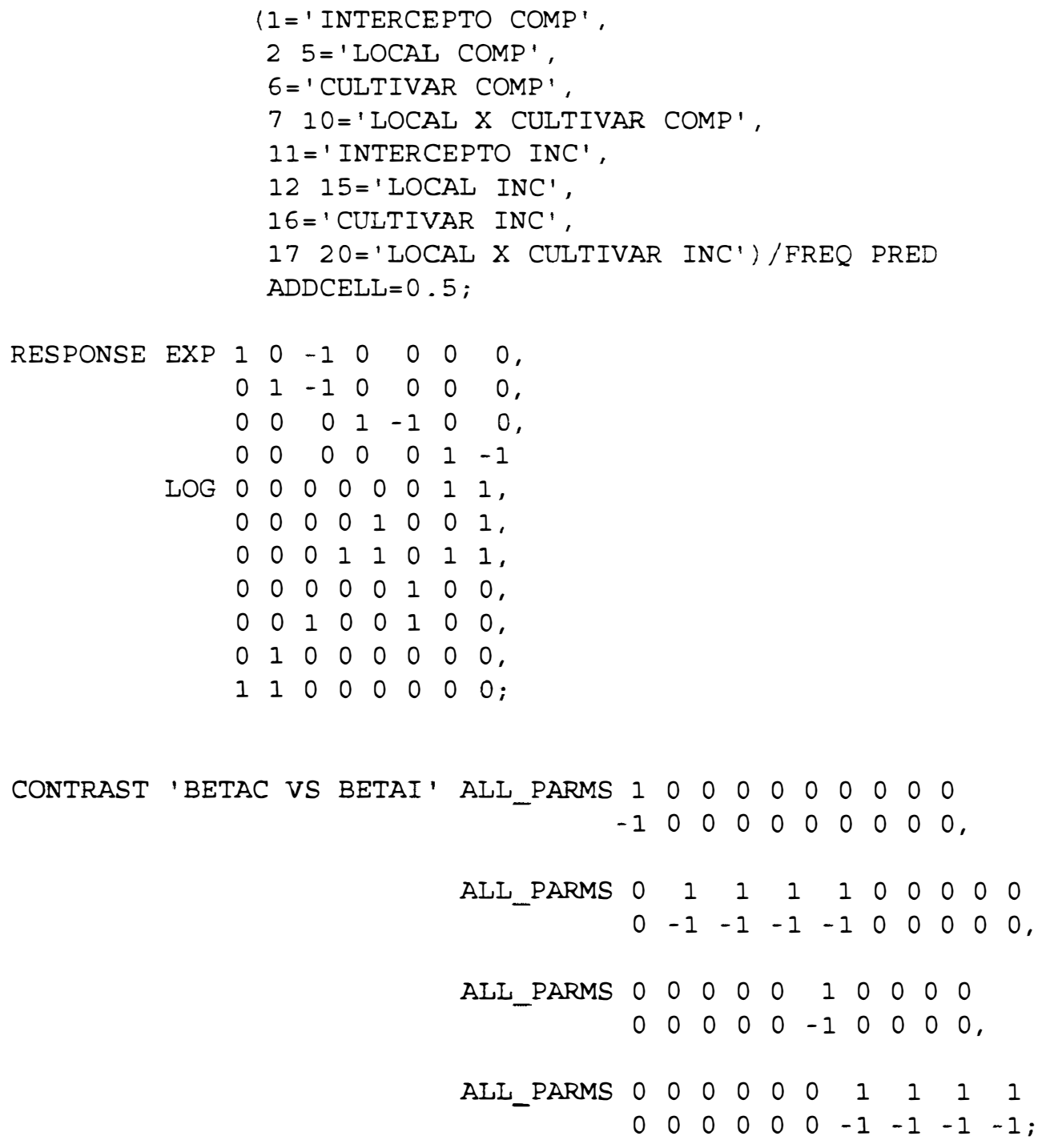

RUN ;

A matriz do delineamento que aparece na declaração MODEL tem sentido análogo à matriz do delineamento apresentada no item 3.4.3.

O contraste 'BETAC VS BETAI' é utilizado para comparar os parâmetros dos dados completos (BETAC) com os parâmetros dos dados incompletos (BETAI). Caso este contraste não seja significativo, então, segundo o teste de Park \& Davis (1993), os dados suportam a condição de MCAR

Para o segundo conjunto de dados basta alterar a declaração INFILE. 


\section{RESULTADOS E DISCUSSĀO}

\subsection{Primeiro conjunto de dados}

\subsubsection{Considerações iniciais}

O primeiro conjunto de dados foi forjado com a intenção de que:

- não houvesse diferença significativa, pelo método de Eskridge (1997), entre as confiabilidades das cultivares 1 e 2 ;

- houvesse diferença significativa, pelo modelo alternativo, entre as confiabilidades das cultivares 1 e 2 .

- qualquer que fosse o modelo adotado, os dados suportariam a condição de MCAR.

\subsubsection{Análise pelo método de Eskridge (1997)}

Através da utilização de qualquer um dos programas apresentados no item 3.2.2, o sistema SAS fornece o seguinte resultado:

CATMOD PROCEDURE

Response: $\mathrm{H} 1$ *H2

Weight Variable: None

Data Set: ESKNI

Frequency Missing: 0

$\begin{array}{lrr}\text { Response Levels }(R)= & 8 \\ \text { Populations } \quad(S)= & 3 \\ \text { Total Frequency }(N)= & 72 \\ \text { Observations (Obs) }= & 72\end{array}$


Aqui são apresentadas características gerais do primeiro conjunto de dados, tais como: são três as populações (anos) e oito níveis de respostas, conforme pode-se ver através dos perfis de respostas apresentados adiante.

\begin{tabular}{|c|c|c|}
\hline \multicolumn{2}{|c|}{ POPULATION } & \multirow{2}{*}{$\begin{array}{c}\text { PROFILES } \\
\text { Sample } \\
\text { Size }\end{array}$} \\
\hline Sample & ANO & \\
\hline & - & $\ldots \ldots$ \\
\hline 1 & 88 & 32 \\
\hline 2 & 89 & 18 \\
\hline 3 & 90 & 22 \\
\hline
\end{tabular}

Os perfis das populações mostram o número de ensaios em cada ano.

\begin{tabular}{crr} 
RESPONSE & \multicolumn{2}{l}{ PROFILES } \\
Response & H1 & H2 \\
1 & -1 & 0 \\
2 & -1 & 1 \\
3 & 0 & -1 \\
4 & 0 & 0 \\
5 & 0 & 1 \\
6 & 1 & -1 \\
7 & 1 & 0 \\
8 & 1 & 1
\end{tabular}

Os perfis das respostas mostram que cada população tem oito categorias de respostas, que são obtidas pelas combinações dos valores de HI com os valores de $\mathrm{H} 2$.

\begin{tabular}{ccccr}
\multicolumn{7}{c}{ RESPONSE FREQUENCIES } \\
Sample & 1 & 2 & 3 & 4 \\
\hdashline 1 & 1 & 4 & 1 & 8 \\
2 & 0 & 4 & 0 & 3 \\
3 & 3 & 2 & 2 & 2
\end{tabular}




\begin{tabular}{|c|c|c|c|c|}
\hline \multirow[b]{2}{*}{ Sample } & \multicolumn{4}{|c|}{ Response Number } \\
\hline & 5 & 6 & 7 & 8 \\
\hline \multicolumn{5}{|c|}{ 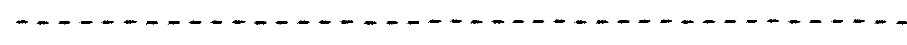 } \\
\hline 1 & 3 & 0 & 2 & 13 \\
\hline 2 & 6 & 1 & 2 & 2 \\
\hline 3 & 3 & 1 & 3 & 6 \\
\hline
\end{tabular}

As frequências das respostas apresentam uma tabela de dupla entrada das amostras (anos) $x$ respostas. Cada casela apresenta o número de ensaios que proporcionou uma dada resposta, em um determinado ano.

\begin{tabular}{lrrr}
\multicolumn{5}{c}{ ANALYSIS-OF-VARIANCE TABLE } \\
SOUrCe & DF & Chi-Square & Prob \\
\hdashline INTERCEPT & 1 & 148.20 & 0.0000 \\
ANO & 2 & 0.40 & 0.8169 \\
CULT & 1 & 2.83 & 0.0924 \\
ANO*CULT & 2 & 2.40 & 0.3019 \\
RESIDUAL & & &
\end{tabular}

Observa-se que não houve efeito significativo de cultivares, bem como de nenhum outro efeito, exceto o de intercepto, ao nível de 0.05 de probabilidade. Este é o nível de significância estatística usado neste trabalho.

Quando utilizado o programa que usa a matriz do delineamento, o sistema SAS fornece o seguinte resultado:

\begin{tabular}{lrrr}
\multicolumn{4}{c}{ ANALYSIS-OF-VARIANCE TABLE } \\
SOUrCe & DF & Chi-Square & Prob \\
\hline INTERCEPTO & 1 & 148.20 & 0.0000 \\
ANO & 2 & 0.40 & 0.8169 \\
CULTIVAR & 1 & 2.83 & 0.0924 \\
ANO X CULTIVAR & 2 & 2.40 & 0.3019 \\
& & &
\end{tabular}




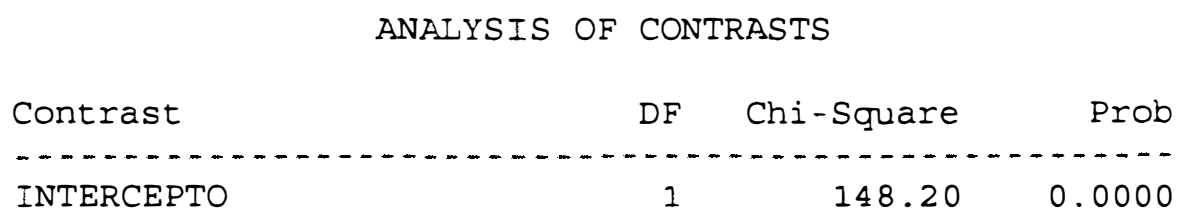

ANALYSIS OF CONTRASTS

Contrast DF Chi-Square Prob

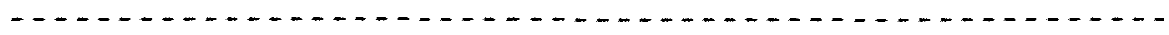

ANO

$20.40 \quad 0.8169$

ANALYSIS OF CONTRASTS
Contrast
DE

ANALYSIS OF CONTRASTS

Contrast DF Chi-Square Prob

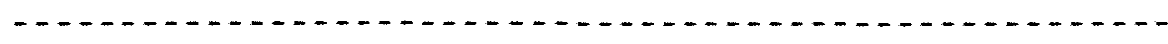

ANO X CULTIVAR

2

$2.40 \quad 0.3019$

Verifica-se que, neste caso, os valores da tabela da análise de variância estão coerentes com os anteriores.

ANALYSIS OF WEIGHTED-LEAST-SQUARES ESTIMATES

\begin{tabular}{lrrrrr} 
Effect & Parameter & Estimate & $\begin{array}{r}\text { Standard } \\
\text { Error }\end{array}$ & $\begin{array}{c}\text { Chi- } \\
\text { Square }\end{array}$ & Prob \\
\hline INTERCEPT & 1 & 0.5718 & 0.0470 & 148.20 & 0.0000 \\
ANO & 2 & 0.0285 & 0.0658 & 0.19 & 0.6646 \\
& 3 & -0.0403 & 0.0656 & 0.38 & 0.5391 \\
CULT & 4 & -0.0715 & 0.0425 & 2.83 & 0.0924 \\
ANO*CULT & 5 & 0.0267 & 0.0494 & 0.29 & 0.5884 \\
& 6 & -0.1029 & 0.0669 & 2.37 & 0.1240
\end{tabular}




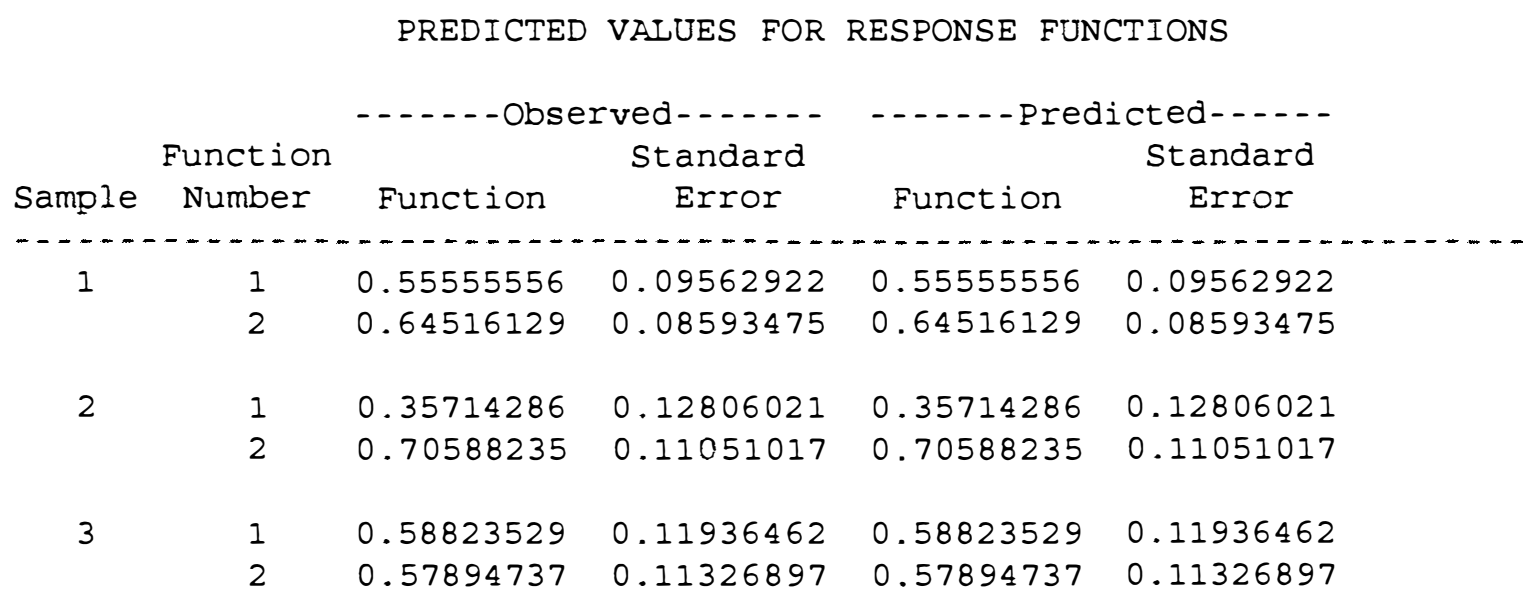

Aqui são apresentados os valores observados e preditos das funções das respostas (confiabilidades), acompanhados dos seus erros padrões. A primeira linha (amostra 1 e função número 1) refere-se à confiabilidade da cultivar 1 , em 1988. A segunda linha (amostra 1 e função número 2) refere-se à confiabilidade da cultivar 2 , em 1988 , e assim por diante.

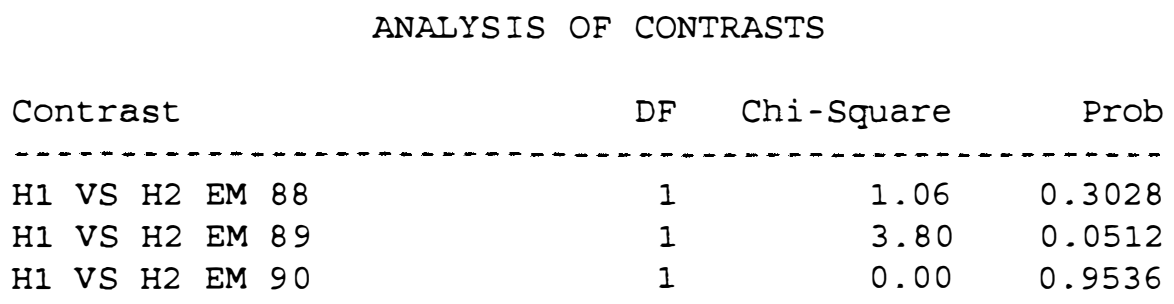

Os contrastes $\mathrm{H} 1$ vs $\mathrm{H} 2$ (confiabilidade da cultivar 1 versus confiabilidade da cultivar 2), dentro de cada ano, revelam que nenhuma cultivar seria indicada para substituir a testemunha, usando o método de Eskridge (1997), que utiliza anos no modelo. 
Os perfis das respostas mostram que cada população tem oito categorias de respostas, que são obtidas pelas combinações dos valores de $\mathrm{Hl}$ com os valores de $\mathrm{H} 2$.

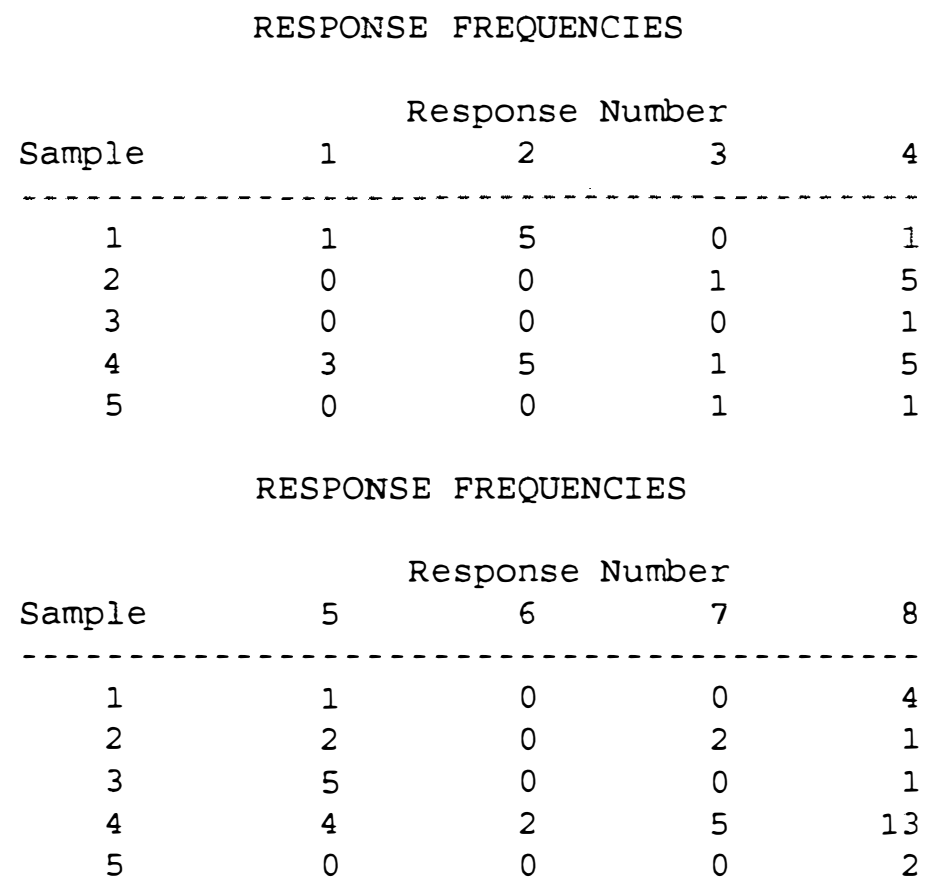

As frequências das respostas apresentam uma tabela de dupla entrada das amostras (locais) $\mathrm{x}$ respostas. Cada casela apresenta o número de ensaios que proporcionou uma dada resposta, em um determinado local.

\begin{tabular}{lrrr}
\multicolumn{4}{c}{ ANALYSIS-OF-VARIANCE TABLE } \\
SOUrCe & DF & Chi-Square & Prob \\
\hline INTERCEPT & 1 & 74.04 & 0.0000 \\
LOCAL & 4 & 11.62 & 0.0204 \\
CULT & 1 & 8.09 & 0.0044 \\
LOCAL ${ }^{2}$ CULT & 4 & 14.59 & 0.0056 \\
RESIDUAL & & &
\end{tabular}


Verifica-se efeito significativo de todas as fontes de variação. Desta forma, o modelo alternativo, que usa locais no modelo, é mais informativo do que o modelo de Eskridge (1997), que usa anos no modelo.

Ressalte-se que se for utilizado o programa que usa a matriz do delineamento no modelo, a qual o próprio sistema SAS fornece, mesmo quando não solicitado ("default"), o resultado da análise de variância é o seguinte:

ANALYSIS-OF-VARIANCE TABLE

$\begin{array}{lcrr}\text { SOurce } & \text { DF } & \text { Chi-Square } & \text { Prob } \\ \text { INTERCEPTO } & 1 & 74.04 & 0.0000 \\ \text { LOCAI } & 2 & 4.07 & 0.1307 \\ \text { CULTIVAR } & 1 & 8.09 & 0.0044 \\ \text { LOCAI X CULTIVAR } & 2 & 5.53 & 0.0631 \\ & & & \end{array}$

ANALYSIS OF CONTRASTS

\begin{tabular}{|c|c|c|}
\hline Contrast & $\mathrm{DF}$ & Chi-Square \\
\hline INTERCEPTO & 1 & 74.04 \\
\hline
\end{tabular}

ANALYSIS OF CONTRASTS

$\begin{array}{lrrr}\text { Contrast } & \text { DF } & \text { Chi-Square } & \text { Prob } \\ \text { LOCAL } & 4 & 11.62 & 0.0204\end{array}$

ANAIYSIS OF CONTRASTS

\begin{tabular}{|c|c|c|}
\hline Contrast & $\mathrm{DF}$ & Chi-Square \\
\hline CULTIVAR & 1 & 8.09 \\
\hline
\end{tabular}


ANALYSIS OF CONTRASTS

\begin{tabular}{|c|c|c|}
\hline ontrast & $D F$ & Chi-Square \\
\hline JCAI X CULTIVAR & 4 & 14.59 \\
\hline
\end{tabular}

Verifica-se que os resultados referentes às fontes de variação local e local $x$ cultivar estão incoerentes, diferindo dos obtidos anteriormente. Desta forma, o uso de contrastes especificos para testar as fontes de variação é uma prática conveniente e necessária.

ANALYSIS OF WEIGHTED-LEAST-SQUARES ESTIMATES

\begin{tabular}{lrrrrr} 
Effect & Parameter & Estimate & $\begin{array}{c}\text { Standard } \\
\text { Error }\end{array}$ & $\begin{array}{r}\text { Chi- } \\
\text { Square }\end{array}$ & Prob \\
\hline INTERCEPT & 1 & 0.5535 & 0.0643 & 74.04 & 0.0000 \\
LOCAL & 2 & 0.1965 & 0.1192 & 2.72 & 0.0992 \\
& 3 & -0.2671 & 0.1013 & 6.95 & 0.0084 \\
& 4 & -0.0535 & 0.1013 & 0.28 & 0.5976 \\
CULT & 5 & 0.0942 & 0.0820 & 1.32 & 0.2509 \\
LOCAL*CULT & 6 & -0.1037 & 0.0364 & 8.09 & 0.0044 \\
& 7 & 0.0203 & 0.0764 & 0.07 & 0.7901 \\
& 8 & 0.0900 & 0.0831 & 1.17 & 0.2787 \\
& 9 & -0.2535 & 0.0755 & 11.27 & 0.0008 \\
& 10 & 0.1227 & 0.0544 & 5.08 & 0.0242
\end{tabular}

ANALYSIS OF CONTRASTS

$\begin{array}{llrr}\text { Contrast } & \text { DF } & \text { Chi-Square } & \text { Prob } \\ \text { H1 VS H2 NO LOCAL A } & 1 & 0.92 & 0.3367 \\ \text { H1 VS H2 NO LOCAL B } & 1 & 0.02 & 0.8876 \\ \text { H1 VS H2 NO LOCAL C } & 1 & 17.50 & 0.0000 \\ \text { H1 VS H2 NO LOCAL M } & 1 & 0.13 & 0.7152 \\ \text { H1 VS H2 NO LOCAL N } & 1 & 1.09 & 0.2963\end{array}$

Observa-se que no local $C$ houve diferença entre a confiabilidade da cultivar 1 e da cultivar 2. Para este local a cultivar 2 deveria ser indicada por apresentar 
maior confiabilidade que a cultivar 1 , conforme pode ser visto pelos valores observados e preditos das funções, dados a seguir.

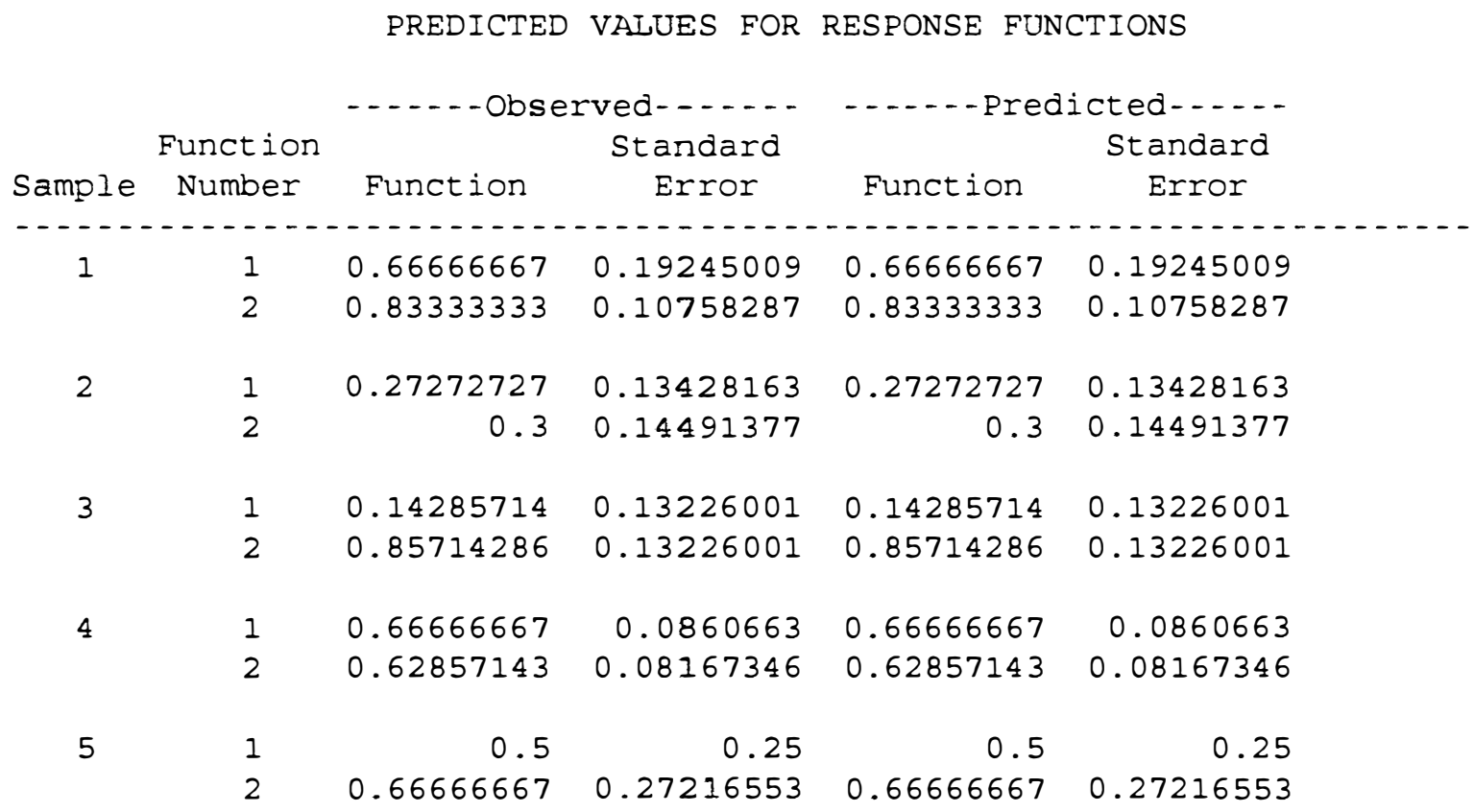

No local C (amostra 3), as confiabilidades das cultivares 2 e 1 foram, aproximadamente, 0,857 (função número 2) e 0,143 (função número 1), respectivamente. Portanto, para este local, a cultivar 2 deveria ser indicada por apresentar alta confiabilidade $(85,7 \%)$.

\subsubsection{Teste do mecanismo de dados incompletos}

Primeiro Caso: Utilizando anos no modelo

Através do programa apresentado no item 3.4.3, o sistema SAS fornece o seguinte resultado: 
CATMOD PROCEDURE

Response: $\mathrm{H} 1{ }^{*} \mathrm{H} 2$

Weight Variable: None

Data Set: PDAl

Frequency Missing: 0

$\begin{array}{llr}\text { Response Levels }(R)= & 8 \\ \text { Populations } & (S)= & 3 \\ \text { Total Frequency }(N)= & 72 \\ \text { Observations (Obs) }= & 72\end{array}$

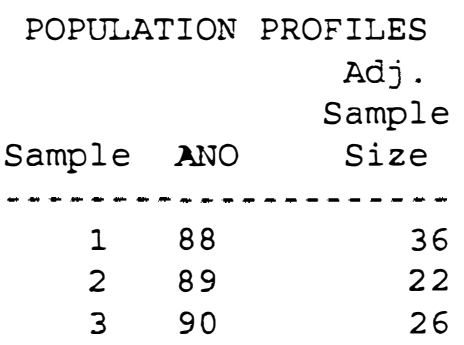

Verifica-se que, neste caso, os tamanhos das amostras foram ajustados em decorrência da utilização da opção ADDCELL $=0.5$ no programa.

\begin{tabular}{ccr} 
RESPONSE PROFILES \\
Response & H1 & H2 \\
\hline 1 & -1 & 0 \\
2 & -1 & 1 \\
3 & 0 & -1 \\
4 & 0 & 0 \\
5 & 0 & 1 \\
6 & 1 & -1 \\
7 & 1 & 0 \\
8 & 1 & 1
\end{tabular}

ADJUSTED RESPONSE FREQUENCIES

\begin{tabular}{ccccr} 
& \multicolumn{4}{c}{ Response Number } \\
Sample & 1 & 2 & 3 & 4 \\
\hline 1 & 1.5 & 4.5 & 1.5 & 8.5 \\
2 & 0.5 & 4.5 & 0.5 & 3.5 \\
3 & 3.5 & 2.5 & 2.5 & 2.5
\end{tabular}




\section{ADJUSTED RESPONSE FREQUENCIES}

\begin{tabular}{ccccr} 
& \multicolumn{4}{c}{ Response Number } \\
Sample & 5 & 6 & 7 & 8 \\
\hline 1 & 3.5 & 0.5 & 2.5 & 13.5 \\
2 & 6.5 & 1.5 & 2.5 & 2.5 \\
3 & 3.5 & 1.5 & 3.5 & 6.5
\end{tabular}

Em decorrência da utilização da opção ADDCELL $=0.5$ no programa, as frequências das amostras foram ajustadas. Todas as análises posteriores são efetuadas baseadas nestas frequências ajustadas.

\section{ANALYSIS-OF-VARIANCE TABLE}

$\begin{array}{lrrr}\text { SOUrCe } & \text { DF } & \text { Chi-Square } & \text { Prob } \\ \text { INTERCEPTO COMP } & 1 & 133.69 & 0.0000 \\ \text { ANOS COMP } & 2 & 1.98 & 0.3708 \\ \text { CULTIVAR COMP } & 1 & 1.30 & 0.2538 \\ \text { ANO X CULTIVAR COMP } & 2 & 1.42 & 0.4918 \\ \text { INTERCEPTO INC } & 1 & 35.27 & 0.0000 \\ \text { ANOS INC } & 2 & 3.71 & 0.1566 \\ \text { CULTIVAR INC } & 1 & 1.42 & 0.2327 \\ \text { ANO X CULTIVAR INC } & 2 & 0.99 & 0.6094\end{array}$

RESIDUAL

0

ANALYSIS OF WEIGHTED-LEAST-SQUARES ESTIMATES

\begin{tabular}{|c|c|c|c|c|c|}
\hline Effect & Parameter & Estimate & $\begin{array}{c}\text { Standard } \\
\text { Error }\end{array}$ & $\begin{array}{l}\text { Chi- } \\
\text { Square }\end{array}$ & Prob \\
\hline \multirow{12}{*}{ MODEL } & 1 & 0.5603 & 0.0485 & 133.69 & 0.0000 \\
\hline & 2 & 0.0290 & 0.0678 & 0.18 & 0.6690 \\
\hline & 3 & -0.0937 & 0.0674 & 1.93 & 0.1648 \\
\hline & 4 & -0.0504 & 0.0442 & 1.30 & 0.253 \\
\hline & 5 & 0.0325 & 0.0508 & 0.41 & 0.522 \\
\hline & 6 & -0.0829 & 0.0699 & 1.41 & 0.235 \\
\hline & 7 & 0.5736 & 0.0966 & 35.27 & 0.0000 \\
\hline & 8 & -0.0736 & 0.1405 & 0.27 & 0.6004 \\
\hline & 9 & 0.2514 & 0.1365 & 3.39 & 0.0656 \\
\hline & 10 & -0.1153 & 0.0966 & 1.42 & 0.2327 \\
\hline & 11 & -0.1347 & 0.1405 & 0.92 & 0.3377 \\
\hline & 12 & 0.0403 & 0.1365 & 0.09 & 0.7680 \\
\hline
\end{tabular}




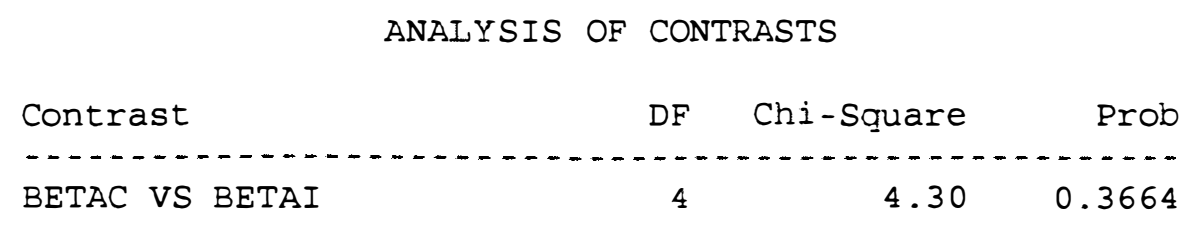

Verifica-se que não há diferença estatisticamente significativa entre os parâmetros do modelo com dados completos e aqueles do modelo com dados incompletos (BETAC VS BETAI). Isto significa que os dados suportam a condição de MCAR Desta forma, a análise incluindo os dados incompletos pode ser implementada sem maiores riscos.

PREDICTED VAIUES FOR RESPONSE FUNCTIONS

\begin{tabular}{|c|c|c|c|c|c|}
\hline \multirow[b]{2}{*}{ Sample } & Function & \multicolumn{2}{|c|}{$\ldots \ldots$. Observed......... } & \multicolumn{2}{|c|}{-.....-Predicted-..... } \\
\hline & Number & Function & Error & Function & Error \\
\hline & 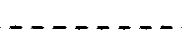 & & $\ldots \ldots$ & $\ldots$ & $\ldots \ldots$ \\
\hline \multirow[t]{4}{*}{1} & 1 & 0.57142857 & 0.09352195 & 0.57142857 & 0.09352195 \\
\hline & 2 & 0.60714286 & 0.09229619 & 0.60714286 & 0.09229619 \\
\hline & 3 & 0.25 & 0.30618622 & 0.25 & 0.30618622 \\
\hline & 4 & 0.75 & 0.1767767 & 0.75 & 0.1767767 \\
\hline \multirow[t]{4}{*}{2} & 1 & 0.33333333 & 0.12171612 & 0.33333333 & 0.12171612 \\
\hline & 2 & 0.6 & 0.12649111 & 0.6 & 0.12649111 \\
\hline & 3 & 0.75 & 0.30618622 & 0.75 & 0.30618622 \\
\hline & 4 & 0.9 & 0.13416408 & 0.9 & 0.13416408 \\
\hline \multirow[t]{4}{*}{3} & 1 & 0.625 & 0.12103073 & 0.625 & 0.12103073 \\
\hline & 2 & 0.625 & 0.12103073 & 0.625 & 0.12103073 \\
\hline & 3 & 0.375 & 0.24206146 & 0.375 & 0.24206146 \\
\hline & 4 & 0.41666667 & 0.20126912 & 0.41666667 & 0.20126912 \\
\hline
\end{tabular}

Caso o contraste BETAC VS BETAI fosse siginificativo, indicando que os dados não são MCAR, os contrastes, apresentados a seguir, seriam utilizados para verificar que parâmetros específicos seriam diferentes entre os dados completos e os dados incompletos. 
ANALYSIS OF CONTRASTS

$\begin{array}{lcrr}\text { Contrast } & \text { DF } & \text { Chi-Square } & \text { Prob } \\ \text { INTERC VS INTERI } & 1 & 0.02 & 0.9021\end{array}$

ANALYSIS OF CONTRASTS

$\begin{array}{lccc}\text { Contrast } & \text { DF } & \text { Chi-Square } & \text { Prob } \\ \text { ANOSC VS ANOSI } & 1 & 2.61 & 0.1063\end{array}$

ANALYSIS OF CONTRASTS

$\begin{array}{lcrr}\text { Contrast } & \text { DF } & \text { Chi-Square } & \text { Prob } \\ \text { CULTC vS CULTI } & 1 & 0.37 & 0.5413\end{array}$

ANALYSIS OF CONTRASTS

Contrast DF Chi-Square Prob

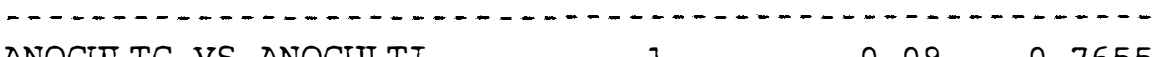

$\begin{array}{llll}\text { ANOCULTC VS ANOCULTI } & 1 & 0.09 & 0.7655\end{array}$

Segundo Caso: Utilizando locais no modelo

Através do programa apresentado no item 3.4.4, o sistema SAS fornece o seguinte resultado:

CATMOD PROCEDURE

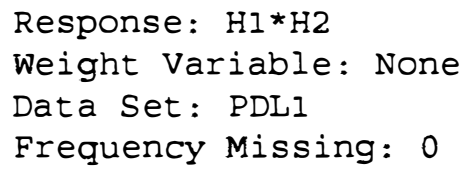

$\begin{array}{llr}\text { Response Levels }(R)= & 8 \\ \text { Populations } & (S)= \\ \text { Total Frequency }(N)= & 72 \\ \text { Observations (Obs) }= & 72\end{array}$




\begin{tabular}{ccr} 
POPULATION $\begin{array}{r}\text { PROFILES } \\
\text { Adj } \\
\text { Sample } \\
\text { Size }\end{array}$ \\
\hline 1 & A & 16 \\
2 & B & 15 \\
3 & C & 11 \\
4 & M & 42 \\
5 & N & 8
\end{tabular}

Verifica-se que, neste caso, os tamanhos das amostras foram ajustados em decorrência da utilização da opção ADDCELL = 0.5 no programa.

$\begin{array}{crr}\text { RESPONSE PROFILES } \\ \text { Response } & \text { H1 } & \text { H2 } \\ 1 & -1 & 0 \\ 2 & -1 & 1 \\ 3 & 0 & -1 \\ 4 & 0 & 0 \\ 5 & 0 & 1 \\ 6 & 1 & -1 \\ 7 & 1 & 0 \\ 8 & 1 & 1\end{array}$

\begin{tabular}{ccccr}
\multicolumn{5}{c}{ ADJUSTED RESPONSE FREQUENCIES } \\
Sample & 1 & 2 & 3 & 4 \\
\hline 1 & 1.5 & 5.5 & 0.5 & 1.5 \\
2 & 0.5 & 0.5 & 1.5 & 5.5 \\
3 & 0.5 & 0.5 & 0.5 & 1.5 \\
4 & 3.5 & 5.5 & 1.5 & 5.5 \\
5 & 0.5 & 0.5 & 1.5 & 1.5
\end{tabular}




\begin{tabular}{crrrr} 
& \multicolumn{4}{c}{ ADJUSTED RESPONSE FREQUENCIES } \\
& \multicolumn{4}{c}{ Response Number } \\
Sample & 5 & 6 & 7 & 8 \\
\hdashline 1 & 1.5 & 0.5 & 0.5 & 4.5 \\
2 & 2.5 & 0.5 & 2.5 & 1.5 \\
3 & 5.5 & 0.5 & 0.5 & 1.5 \\
4 & 4.5 & 2.5 & 5.5 & 13.5 \\
5 & 0.5 & 0.5 & 0.5 & 2.5
\end{tabular}

Em decorrência da utilização da opção ADDCELL $=0.5$ no programa, as frequências das amostras foram ajustadas. Todas as análises posteriores são efetuadas baseadas nestas frequências ajustadas.

\begin{tabular}{|c|c|c|c|}
\hline Source & $D F$ & Chi-Square & Prob \\
\hline 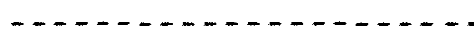 & $\cdots$ & $\ldots .$. & - \\
\hline INTERCEPTO COMP & 1 & 93.81 & 0.0000 \\
\hline LOCAL COMP & 2 & 2.92 & 0.2319 \\
\hline CUITIVAR COMP & 1 & 2.68 & 0.1015 \\
\hline LOCAL X CULTIVAR COMP & 2 & 2.04 & 0.3597 \\
\hline INTERCEPTO INC & 1 & 16.31 & 0.0001 \\
\hline LOCAI INC & 2 & 0.88 & 0.6441 \\
\hline CULTIVAR INC & 1 & 0.39 & 0.5348 \\
\hline LOCAL X CULTIVAR INC & 2 & 0.31 & 0.8560 \\
\hline RESIDUAL & 0 & & \\
\hline
\end{tabular}

Observa-se que alguns valores da tabela da análise de variância estão incoerentes. Caso fosse de interesse, poder-se-ia construir contrastes especificos para a obtenção de valores coerentes. Mas, neste caso, estes valores em nada invalidam as análises posteriores. Por este motivo, não foram construidos contrastes especificos. 


\begin{tabular}{|c|c|c|c|c|c|}
\hline Effect & Parameter & Estimate & $\begin{array}{c}\text { Standard } \\
\text { Error }\end{array}$ & $\begin{array}{l}\text { Chi- } \\
\text { Square }\end{array}$ & Prob \\
\hline \multirow[t]{20}{*}{ MODEL } & 1 & 0.5518 & 0.0570 & 93.81 & 0.0000 \\
\hline & 2 & 0.1357 & 0.1211 & 1.26 & 0.2624 \\
\hline & 3 & -0.2184 & 0.0956 & 5.21 & 0.0224 \\
\hline & 4 & -0.0518 & 0.0938 & 0.30 & 0.5812 \\
\hline & 5 & 0.0862 & 0.0790 & 1.19 & 0.2753 \\
\hline & 6 & -0.0646 & 0.0395 & 2.68 & 0.1015 \\
\hline & 7 & 0.00211 & 0.0771 & 0.00 & 0.9782 \\
\hline & 8 & 0.0646 & 0.0822 & 0.62 & 0.4322 \\
\hline & 9 & -0.2132 & 0.0867 & 6.04 & 0.0140 \\
\hline & 10 & 0.0818 & 0.0577 & 2.01 & 0.1563 \\
\hline & 11 & 0.5022 & 0.1244 & 16.31 & 0.0001 \\
\hline & 12 & 0.1407 & 0.2379 & 0.35 & 0.5542 \\
\hline & 13 & -0.1272 & 0.2589 & 0.24 & 0.6233 \\
\hline & 14 & -0.00218 & 0.3008 & 0.00 & 0.9942 \\
\hline & 15 & 0.1159 & 0.1680 & 0.48 & 0.4903 \\
\hline & 16 & -0.0772 & 0.1244 & 0.39 & 0.5348 \\
\hline & 17 & -0.0657 & 0.2379 & 0.08 & 0.7825 \\
\hline & 18 & -0.0478 & 0.2589 & 0.03 & 0.8535 \\
\hline & 19 & 0.0772 & 0.3008 & 0.07 & 0.7975 \\
\hline & 20 & 0.0841 & 0.1680 & 0.25 & 0.6165 \\
\hline
\end{tabular}

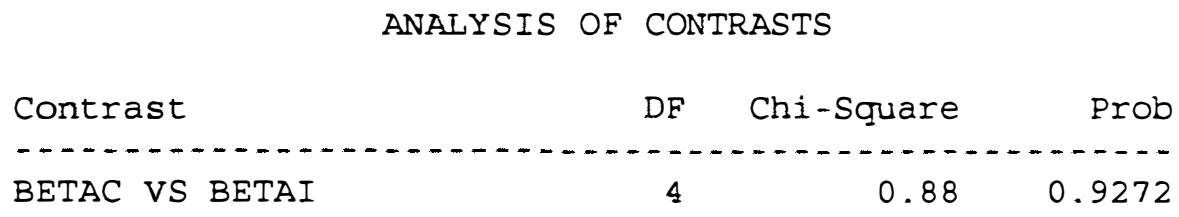

Verifica-se que não há diferença estatisticamente significativa entre os parâmetros do modelo com dados completos e aqueles do modelo com dados incompletos. Desta forma, a análise incluindo os dados incompletos pode ser implementada sem maiores riscos. 


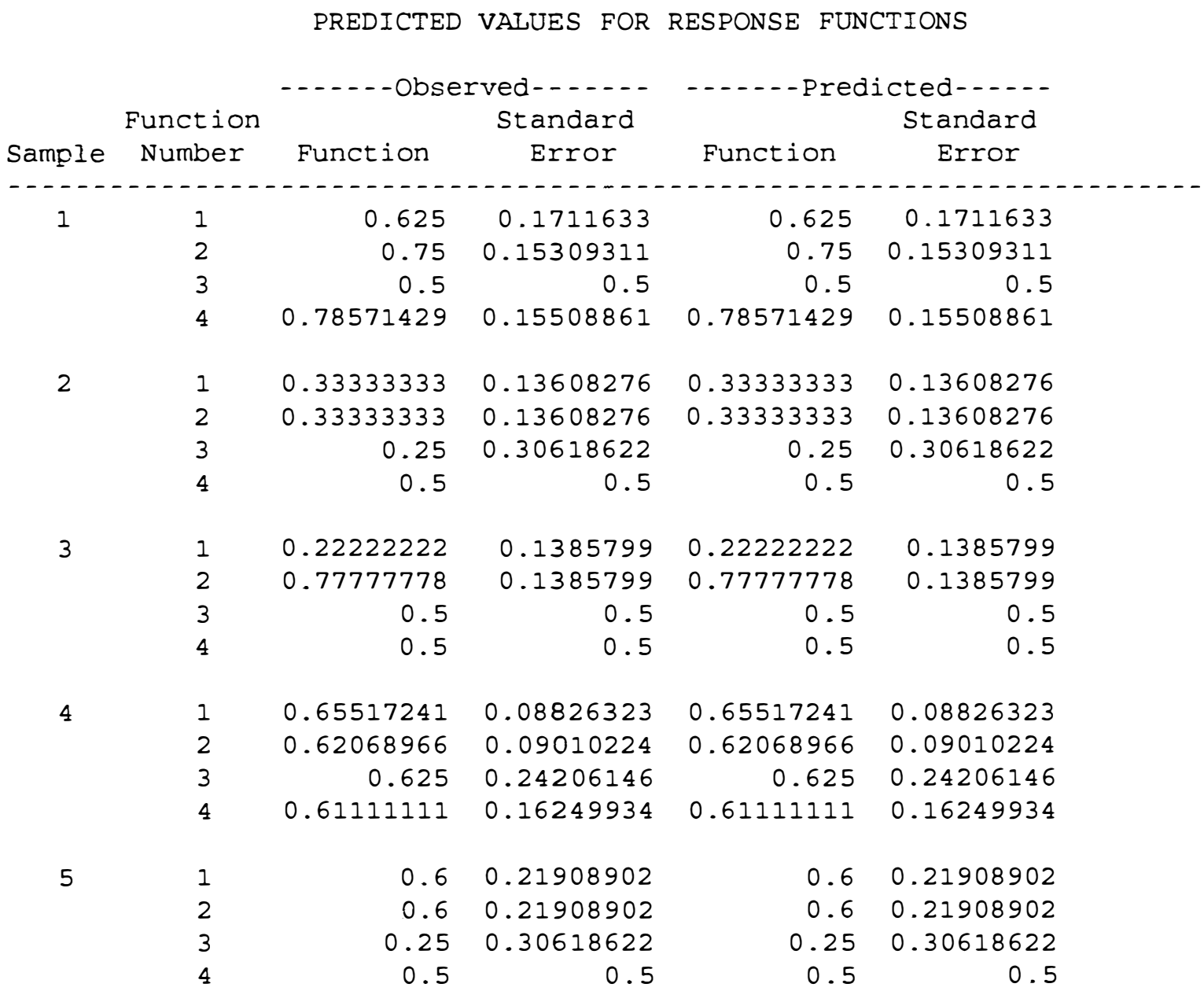

\subsection{Segundo conjunto de dados}

\subsubsection{Considerações iniciais}

O segundo conjunto de dados foi forjado com a intenção de que:

- não houvesse diferença significativa, pelo método de Eskridge (1997), entre as confiabilidades das cultivares 1 e 2 ;

- houvesse diferença significativa, pelo modelo alternativo, entre as confiabilidades das cultivares 1 e 2 . 
- se fosse usado anos no modelo, os dados não suportariam a condição de MCAR, mas, se fosse utilizado o modelo alternativo, que usa locais no modelo, os dados suportariam a condição de MCAR.

\subsubsection{Análise pelo método de Eskridge (1997)}

Através da utilização de qualquer um dos programas apresentados no item 3.2.2, o sistema SAS fornece o seguinte resultado:

Response: $\mathrm{HI}$ *H2

Weight Variable: None Data Set: ESKN2

Frequency Missing: 0
CATMOD PROCEDURE

$\begin{array}{lrr}\text { Response Levels }(R)= & 8 \\ \text { Populations } & (S)= & 3 \\ \text { Total Frequency }(N)= & 72 \\ \text { Observations (Obs) }= & 72\end{array}$

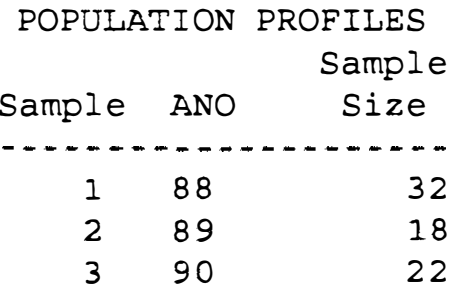

RESPONSE PROFILES

$\begin{array}{crr}\text { Response } & \mathrm{H} 1 & \mathrm{H} 2 \\ 1 & -1 & 0 \\ 2 & -1 & 1 \\ 3 & 0 & -1 \\ 4 & 0 & 0 \\ 5 & 0 & 1 \\ 6 & 1 & -1 \\ 7 & 1 & 0 \\ 8 & 1 & 1\end{array}$




\begin{tabular}{ccccc}
\multicolumn{5}{c}{ RESPONSE FREQUENCIES } \\
Sample & 1 & Response Number \\
\hline 1 & 2 & 3 & 4 \\
2 & 1 & 4 & 1 & 8 \\
3 & 0 & 4 & 0 & 3 \\
& 3 & 2 & 2 & 2
\end{tabular}

RESPONSE FREQUENCIES

\begin{tabular}{ccccr} 
& \multicolumn{4}{c}{ Response Number } \\
Sample & 5 & 6 & 7 & 8 \\
\hline 1 & 1 & 0 & 4 & 13 \\
2 & 2 & 1 & 6 & 2 \\
3 & 1 & 1 & 5 & 6
\end{tabular}

ANALYSIS-OF-VARIANCE TABLE

\begin{tabular}{lccc} 
Source & DF & Chi-Square & Prob \\
\hline INTERCEPT & 1 & 149.86 & 0.0000 \\
ANO & 2 & 0.18 & 0.9130 \\
CULT & 1 & 3.22 & 0.0728 \\
ANO*CULT & 2 & 1.29 & 0.5244
\end{tabular}

RESIDUAL

0

Observa-se que não houve efeito significativo de cultivares, bem como de nenhum outro efeito, exceto o de intercepto.

Quando utilizado o programa que usa a matriz do delineamento, o sistema SAS fornece o seguinte resultado:

ANALYSIS-OF-VARIANCE TABLE

\begin{tabular}{lrrr} 
Source & DF & Chi-Square & Prob \\
\hline INTERCEPTO & 1 & 149.86 & 0.0000 \\
ANOS & 2 & 0.18 & 0.9130 \\
CULTIVAR & 1 & 3.22 & 0.0728 \\
ANO X CULTIVAR & 2 & 1.29 & 0.5244
\end{tabular}




ANALYSIS OF CONTRASTS
Contrast
DF

ANALYSIS OF CONTRASTS

$\begin{array}{llrr}\text { Contrast } & \text { DF } & \text { Chi-Square } & \text { Prob } \\ \text { ANO } & 2 & 0.18 & 0.9130\end{array}$

ANALYSIS OF CONTRASTS

$\begin{array}{lcrr}\text { Contrast } & \text { DF } & \text { Chi-Square } & \text { Prob } \\ \text { CULTIVAR } & 1 & 3.22 & 0.0728\end{array}$

ANALYSIS OF CONTRASTS

$\begin{array}{lcrr}\text { Contrast } & \text { DF } & \text { Chi-Square } & \text { Prob } \\ \text { ANO X CULTIVAR } & 2 & 1.29 & 0.5244\end{array}$

Verifica-se que, neste caso, os valores da tabela da análise de variância estão coerentes com os anteriores.

ANALYSIS OF WEIGHTED-LEAST-SQUARES ESTIMATES

\begin{tabular}{lrrrrr} 
Effect & Parameter & Standard & \multicolumn{1}{c}{$\begin{array}{c}\text { Chi- } \\
\text { Equare }\end{array}$} \\
\hline INTERCEPT & 1 & 0.5839 & 0.0477 & 149.86 & 0.0000 \\
ANO & 2 & 0.0213 & 0.0664 & 0.10 & 0.7489 \\
& 3 & -0.0272 & 0.0677 & 0.16 & 0.6882 \\
CULT & 4 & 0.0756 & 0.0421 & 3.22 & 0.0728 \\
ANO*CULT & 5 & -0.0511 & 0.0489 & 1.09 & 0.2958 \\
& 6 & 0.0106 & 0.0681 & 0.02 & 0.8769
\end{tabular}


PREDICTED VALUES FOR RESPONSE FUNCTIONS

\begin{tabular}{|c|c|c|c|c|c|}
\hline \multicolumn{2}{|r|}{ Function } & \multicolumn{2}{|c|}{-...-- Observed-..... } & \multicolumn{2}{|c|}{$\ldots-\ldots$ - Predicted-.... } \\
\hline Sample & Number & Function & Error & Function & Error \\
\hline \multicolumn{6}{|c|}{ 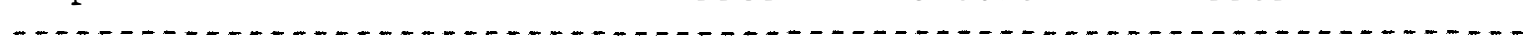 } \\
\hline \multirow[t]{2}{*}{1} & 1 & 0.62962963 & 0.0929349 & 0.62962963 & 0.0929349 \\
\hline & 2 & 0.58064516 & 0.08862687 & 0.58064516 & 0.08862687 \\
\hline \multirow[t]{2}{*}{2} & 1 & 0.64285714 & 0.12806021 & 0.64285714 & 0.12806021 \\
\hline & 2 & 0.47058824 & 0.12105782 & 0.47058824 & 0.12105782 \\
\hline \multirow[t]{2}{*}{3} & 1 & 0.70588235 & 0.11051017 & 0.70588235 & 0.11051017 \\
\hline & 2 & 0.47368421 & 0.11454888 & 0.47368421 & 0.11454888 \\
\hline
\end{tabular}

ANALYSIS OF CONTRASTS

$\begin{array}{llll}\text { Contrast } & \text { DF } & \text { Chi-Square } & \text { Prob } \\ \text { HI VS H2 EM } 88 & 1 & 0.33 & 0.5681 \\ \text { HI VS H2 EM } 89 & 1 & 0.86 & 0.3533 \\ \text { HI VS H2 EM } 90 & 1 & 2.44 & 0.1179\end{array}$

Os contrastes $\mathrm{H} 1$ vs $\mathrm{H} 2$ (confiabilidade da cultivar 1 versus confiabilidade da cultivar 2), dentro de cada ano, revelam que nenhuma cultivar seria indicada para substituir a testemunha.

\subsubsection{Análise pelo modelo alternativo}

Através da utilização de qualquer um dos programas apresentados no item 3.3.2, o sistema SAS fornece o seguinte resultado:

CATMOD PROCEDURE

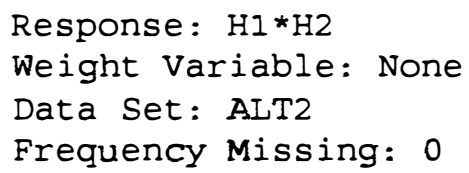

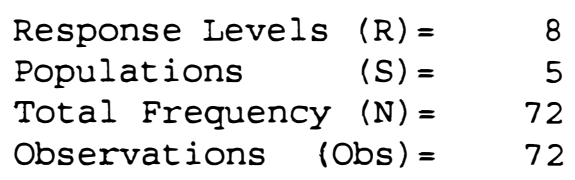




\begin{tabular}{ccr}
$\begin{array}{c}\text { POPULATION PROFILES } \\
\text { Sample } \\
\text { Sample }\end{array}$ \\
\hline 1 & LOCAL & A \\
2 & B & 12 \\
3 & C & 7 \\
4 & M & 38 \\
5 & N & 4
\end{tabular}

RESPONSE PROFILES

Response $\mathrm{H} 1 \mathrm{H} 2$

..............

$\begin{array}{lll}1 & -1 & 0\end{array}$

$\begin{array}{lll}2 & -1 & 1\end{array}$

$\begin{array}{lll}3 & 0 & -1\end{array}$

4000

$\begin{array}{lll}5 & 0 & 1\end{array}$

$\begin{array}{lll}6 & 1 & -1\end{array}$

$\begin{array}{lll}7 & 1 & 0\end{array}$

$\begin{array}{lll}8 & 1 & 1\end{array}$

RESPONSE FREQUENCIES

\begin{tabular}{ccccc} 
& \multicolumn{4}{c}{ Response Number } \\
Sample & 1 & 2 & 3 & 4 \\
\hline 1 & 1 & 5 & 0 & 1 \\
2 & 0 & 0 & 1 & 5 \\
3 & 0 & 0 & 0 & 1 \\
4 & 3 & 5 & 1 & 5 \\
5 & 0 & 0 & 1 & 1
\end{tabular}

RESPONSE FREQUENCIES

\begin{tabular}{ccccr} 
& \multicolumn{4}{c}{ Response Number } \\
Sample & 5 & .6 & 7 & 8 \\
\hline 1 & 0 & 0 & 1 & 4 \\
2 & 1 & 0 & 3 & 1 \\
3 & 2 & 0 & 3 & 1 \\
4 & 1 & 2 & 8 & 13 \\
5 & 0 & 0 & 0 & 2
\end{tabular}


ANALYSIS-OF-VARIANCE TABLE

\begin{tabular}{lrrr} 
SOurCe & DF & Chi-Square & Prob \\
\hline INTERCEPT & 1 & 78.36 & 0.0000 \\
LOCAL & 4 & 13.77 & 0.0081 \\
CULT & 1 & 1.07 & 0.3017 \\
LOCAL*CULT & 4 & 4.55 & 0.3370 \\
RESIDUAL & & &
\end{tabular}

Verifica-se que há efeito significativo de locais e do intercepto.

Ressalte-se que se for utilizado o programa que usa a matriz do delineamento no modelo, a qual o próprio sistema SAS fornece, mesmo quando não solicitado ("default"), o resultado da análise de variância é o seguinte:

\begin{tabular}{lcrr}
\multicolumn{4}{c}{ ANALYSIS-OF-VARIANCE TABLE } \\
SOUrCe & DF & Chi-Square & Prob \\
\hline INTERCEPTO & 1 & 78.36 & 0.0000 \\
LOCAI & 2 & 5.31 & 0.0704 \\
CULTIVAR & 1 & 1.07 & 0.3017 \\
LOCAI X CULTIVAR & 2 & 1.43 & 0.4902 \\
RESIDUAL & & &
\end{tabular}

ANALYSIS OF CONTRASTS

$\begin{array}{llrr}\text { Contrast } & \text { DF } & \text { Chi-Square } & \text { Prob } \\ \text { LOCAL } & 4 & 13.77 & 0.0081\end{array}$


ANALYSIS OF CONTRASTS

\begin{tabular}{|c|c|c|}
\hline Contrast & $\mathrm{DF}$ & Chi-Square \\
\hline CULTIVAR & 1 & 1.07 \\
\hline
\end{tabular}

ANALYSIS OF CONTRASTS

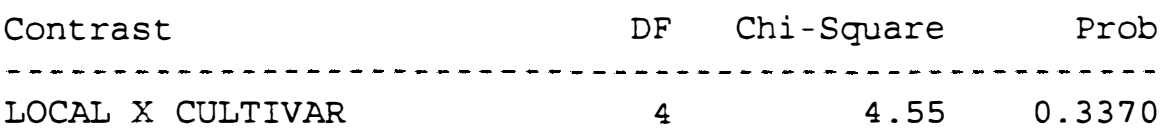

Verifica-se que os resultados referentes às fontes de variação local e local $\mathrm{x}$ cultivar estão incoerentes, diferindo dos obtidos anteriormente. Desta forma, o uso de contrastes específicos para testar as fontes de variação é uma prática conveniente e necessária.

\begin{tabular}{|c|c|c|c|c|c|}
\hline Effect & Parameter & Estimate & $\begin{array}{c}\text { Standard } \\
\text { Error }\end{array}$ & $\begin{array}{l}\text { Chi- } \\
\text { Square }\end{array}$ & Prob \\
\hline$\ldots$ & & $\ldots \ldots$ & & $\ldots \ldots$ & $\cdots$ \\
\hline INTERCEPT & 1 & 0.5623 & 0.0635 & 78.36 & 0.0000 \\
\hline \multirow[t]{4}{*}{ LOCAL } & 2 & 0.2294 & 0.1124 & 4.17 & 0.0413 \\
\hline & 3 & -0.2805 & 0.1007 & 7.76 & 0.0053 \\
\hline & 4 & -0.0623 & 0.1008 & 0.38 & 0.5364 \\
\hline & 5 & 0.0924 & 0.0812 & 1.30 & 0.2549 \\
\hline CULT & 6 & 0.0447 & 0.0433 & 1.07 & 0.3017 \\
\hline \multirow[t]{4}{*}{ LOCAL * CULT } & 7 & -0.00303 & 0.0701 & 0.00 & 0.9655 \\
\hline & 8 & 0.0371 & 0.0830 & 0.20 & 0.6546 \\
\hline & 9 & 0.0267 & 0.1294 & 0.04 & 0.8363 \\
\hline & 10 & 0.0672 & 0.0568 & 1.40 & 0.2370 \\
\hline
\end{tabular}

ANALYSIS OF . CONTRASTS

$\begin{array}{lllr}\text { Contrast } & \text { DF } & \text { Chi-Square } & \text { Prob } \\ \text { H1 VS H2 NO LOCAL A } & 1 & 0.34 & 0.5582 \\ \text { H1 VS H2 NO LOCAL B } & 1 & 0.80 & 0.3706 \\ \text { H1 VS H2 NO LOCAL C } & 1 & 0.21 & 0.6500 \\ \text { H1 VS H2 NO LOCAL M } & 1 & 5.54 & 0.0186 \\ \text { H1 VS H2 NO LOCAL N } & 1 & 1.09 & 0.2963\end{array}$


Verifica-se, pelo contraste $\mathrm{H} 1$ vs $\mathrm{H} 2$ no local $\mathrm{M}$, que há diferença entre a confiabilidade da cultivar 1 e da cultivar 2 , sendo, neste caso, maior a confiabilidade da cultivar 1, conforme pode ser visto pelas funções observadas e estimadas dadas a seguir.

\begin{tabular}{|c|c|c|c|c|c|}
\hline \multirow[b]{3}{*}{ Sample } & \multirow{3}{*}{$\begin{array}{l}\text { Function } \\
\text { Number }\end{array}$} & PREDICTED & VALUES FOR & \multicolumn{2}{|c|}{ RESPONSE FUNCTIONS } \\
\hline & & \multicolumn{2}{|c|}{ - . - Observed-..... } & ....... Predicted...... & $\begin{array}{l}\text { icted-..... } \\
\text { Standard }\end{array}$ \\
\hline & & Function & Error & Function & Error \\
\hline$\ldots \ldots$ & & $\ldots \ldots$ & $\ldots \ldots$ & $\ldots \ldots \ldots$ & $\ldots \ldots \ldots$ \\
\hline \multirow[t]{2}{*}{1} & 1 & 0.83333333 & 0.15214515 & 0.83333333 & 0.15214515 \\
\hline & 2 & 0.75 & 0.125 & 0.75 & 0.125 \\
\hline \multirow[t]{2}{*}{2} & 1 & 0.36363636 & 0.14504073 & 0.36363636 & 0.14504073 \\
\hline & 2 & 0.2 & 0.12649111 & 0.2 & 0.12649111 \\
\hline \multirow[t]{2}{*}{3} & 1 & 0.57142857 & 0.18704391 & 0.57142857 & 0.18704391 \\
\hline & 2 & 0.42857143 & 0.18704391 & 0.42857143 & 0.18704391 \\
\hline \multirow[t]{2}{*}{4} & 1 & 0.76666667 & 0.07722022 & 0.76666667 & 0.07722022 \\
\hline & 2 & 0.54285714 & 0.08420439 & 0.54285714 & 0.08420439 \\
\hline \multirow[t]{2}{*}{5} & 1 & 0.5 & 0.25 & 0.5 & 0.25 \\
\hline & 2 & 0.66666667 & 0.27216553 & 0.66666667 & 0.27216553 \\
\hline
\end{tabular}

No local M (amostra 4), as confiabilidades das cultivares 1 e 2 foram, aproximadamente, 0,767 (função número 1) e 0,543 (função número 2), respectivamente. Portanto, para este local, a cultivar 1 deveria ser indicada por apresentar alta confiabilidade $(76,7 \%)$.

\subsubsection{Teste do mecanismo de dados incompletos}

Primeiro Caso: Utilizando anos no modelo

Através do programa apresentado no item 3.4.3, o sistema SAS fornece o seguinte resultado: 
CATMOD PROCEDURE

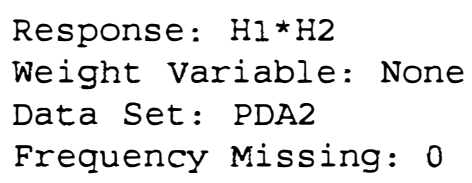

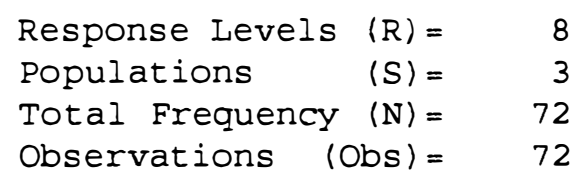

Verifica-se que, neste caso, os tamanhos das amostras foram ajustados em decorrência da utilização da opção $\mathrm{ADDCELL}=0.5$ no programa.

\begin{tabular}{crr} 
RESPONSE PROFILES \\
Response & HI & H2 \\
\hdashline 1 & -1 & 0 \\
2 & -1 & 1 \\
3 & 0 & -1 \\
4 & 0 & 0 \\
5 & 0 & 1 \\
6 & 1 & -1 \\
7 & 1 & 0 \\
8 & 1 & 1
\end{tabular}

ADJUSTED RESPONSE FREQUENCIES

\begin{tabular}{ccccr} 
& \multicolumn{4}{c}{ Response Number } \\
Sample & 1 & 2 & 3 & 4 \\
\hline 1 & 1.5 & 4.5 & 1.5 & 8.5 \\
2 & 0.5 & 4.5 & 0.5 & 3.5 \\
3 & 3.5 & 2.5 & 2.5 & 2.5
\end{tabular}


ADJUSTED RESPONSE FREQUENCIES

\begin{tabular}{ccccr} 
& \multicolumn{4}{c}{ Response Number } \\
Sample & 5 & 6 & 7 & 8 \\
\hline 1 & 1.5 & 0.5 & 4.5 & 13.5 \\
2 & 2.5 & 1.5 & 6.5 & 2.5 \\
3 & 1.5 & 1.5 & 5.5 & 6.5
\end{tabular}

Em decorrência da utilização da opção $\mathrm{ADDCELL}=0.5$ no programa, as frequências das amostras foram ajustadas. Todas as análises posteriores são efetuadas baseadas nestas frequências ajustadas.

ANALYSIS-OF-VARIANCE TABLE

$\begin{array}{lrrr}\text { SOurCe } & \text { DF } & \text { Chi-Square } & \text { Prob } \\ \text { INTERCEPTO COMP } & 1 & 133.69 & 0.0000 \\ \text { ANO COMP } & 2 & 1.98 & 0.3708 \\ \text { CULTIVAR COMP } & 1 & 5.90 & 0.0151 \\ \text { ANO X CULTIVAR COMP } & 2 & 1.05 & 0.5906 \\ \text { INTERCEPTO INC } & 1 & 35.27 & 0.0000 \\ \text { ANO INC } & 2 & 3.71 & 0.1566 \\ \text { CULTIVAR INC } & 1 & 1.42 & 0.2327 \\ \text { ANO X CULTIVAR INC } & 2 & 0.99 & 0.6094\end{array}$

RESIDUAL

0

ANALYSIS OF WEIGHTED-LEAST-SQUARES ESTIMATES

\begin{tabular}{|c|c|c|c|c|c|}
\hline Effect & Parameter & Estimate & $\begin{array}{c}\text { Standard } \\
\text { Error }\end{array}$ & $\begin{array}{l}\text { Chi- } \\
\text { Square }\end{array}$ & Prob \\
\hline & $\ldots$ & $\cdots$ & & $\cdots-$ & -- \\
\hline \multirow[t]{12}{*}{ MODEL } & 1 & 0.5603 & 0.0485 & 133.69 & 0.0000 \\
\hline & 2 & 0.0290 & 0.0678 & 0.18 & 0.6690 \\
\hline & 3 & -0.0937 & 0.0674 & 1.93 & 0.1648 \\
\hline & 4 & 0.1040 & 0.0428 & 5.90 & 0.0151 \\
\hline & 5 & -0.0504 & 0.0493 & 1.04 & 0.3071 \\
\hline & 6 & 0.0294 & 0.0691 & 0.18 & 0.6707 \\
\hline & 7 & 0.5736 & 0.0966 & 35.27 & 0.0000 \\
\hline & 8 & -0.0736 & 0.1405 & 0.27 & 0.6004 \\
\hline & 9 & 0.2514 & 0.1365 & 3.39 & 0.0656 \\
\hline & 10 & -0.1153 & 0.0966 & 1.42 & 0.2327 \\
\hline & 11 & -0.1347 & 0.1405 & 0.92 & 0.3377 \\
\hline & 12 & 0.0403 & 0.1365 & 0.09 & 0.7680 \\
\hline
\end{tabular}




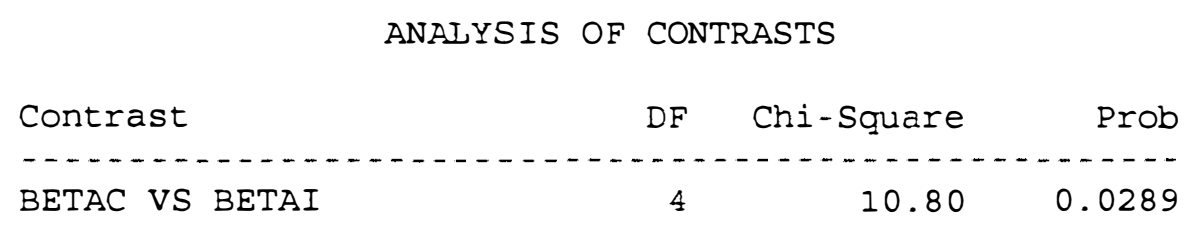

Verifica-se que há diferença estatisticamente significativa entre os parâmetros do modelo com dados completos e aqueles do modelo com dados incompletos (BETAC VS BETAI). Desta forma, a análise incluindo os dados incompletos não deveria ser implementada, pois pode ser uma análise viesada, já que pelo teste de Park \& Davis (1993) os dados não são MCAR.

\begin{tabular}{|c|c|c|c|c|c|}
\hline \multirow[b]{3}{*}{ Sample } & \multirow{3}{*}{$\begin{array}{l}\text { Function } \\
\text { Number }\end{array}$} & PREDICTED & VALUES FOR & \multicolumn{2}{|c|}{ RESPONSE FUNCTIONS } \\
\hline & & \multicolumn{2}{|c|}{$\ldots \ldots$ - Observed -...... } & \multicolumn{2}{|c|}{ - . . - Predicted-.... } \\
\hline & & Function & Error & Function & Error \\
\hline 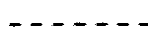 & & $\cdots$ & $-\ldots$ & 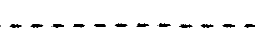 & $\ldots \ldots$ \\
\hline \multirow[t]{4}{*}{1} & 1 & 0.64285714 & 0.09055224 & 0.64285714 & 0.09055224 \\
\hline & 2 & 0.53571429 & 0.09424976 & 0.53571429 & 0.09424976 \\
\hline & 3 & 0.25 & 0.30618622 & 0.25 & 0.30618622 \\
\hline & 4 & 0.75 & 0.1767767 & 0.75 & 0.1767767 \\
\hline \multirow[t]{4}{*}{2} & 1 & 0.6 & 0.12649111 & 0.6 & 0.12649111 \\
\hline & 2 & 0.33333333 & 0.12171612 & 0.33333333 & 0.12171612 \\
\hline & 3 & 0.75 & 0.30618622 & 0.75 & 0.30618622 \\
\hline & 4 & 0.9 & 0.13416408 & 0.9 & 0.13416408 \\
\hline \multirow[t]{8}{*}{3} & 1 & 0.75 & 0.10825318 & 0.75 & 0.10825318 \\
\hline & 2 & 0.5 & 0.125 & 0.5 & 0.125 \\
\hline & 3 & 0.375 & 0.24206146 & 0.375 & 0.24206146 \\
\hline & 4 & 0.41666667 & 0.20126912 & 0.41666667 & 0.20126912 \\
\hline & \multicolumn{5}{|c|}{ ANALYSIS OF CONTRASTS } \\
\hline & \multicolumn{3}{|c|}{ Contrast } & Chi-Squ & Prob \\
\hline & \multirow{2}{*}{\multicolumn{3}{|c|}{ INTERC VS INTER }} & $\ldots \ldots \ldots$ & $\ldots \ldots$ \\
\hline & & & & 1 & 0.9021 \\
\hline
\end{tabular}


ANALYSIS OF CONTRASTS

$\begin{array}{lccc}\text { Contrast } & \text { DF } & \text { Chi-Square } & \text { Prob } \\ \text { ANOSC VS ANOSI } & 1 & 2.61 & 0.1063\end{array}$

ANALYSIS OF CONTRASTS

$\begin{array}{lccr}\text { Contrast } & \text { DF } & \text { Chi-Square } & \text { Prob } \\ \text { CULTC VS CULTI } & 1 & 4.31 & 0.0380\end{array}$

Verifica-se, pelo contraste CULTC VS CULTI, que o efeito de cultivares com dados completos é significativamente diferente do efeito de cultivares com dados incompletos.

\begin{tabular}{llrr}
\multicolumn{5}{c}{ ANALYSIS OF CONTRASTS } \\
Contrast & DF & Chi-Square & Prob \\
\hline ANOCULTC VS ANOCULTI & 1 & 0.25 & 0.6156
\end{tabular}

Segundo Caso: Utilizando locais no modelo

Através do programa apresentado no item 3.4.3, o sistema SAS fornece o seguinte resultado:

CATMOD PROCEDURE

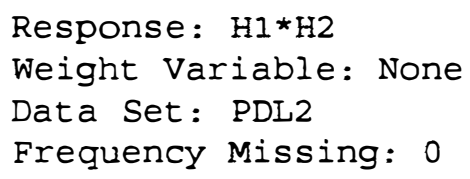

$\begin{array}{lrr}\text { Response Levels }(R)= & 8 \\ \text { Populations } & (S)= & 5 \\ \text { Total Frequency }(N)= & 72 \\ \text { Observations (Obs) }= & 72\end{array}$




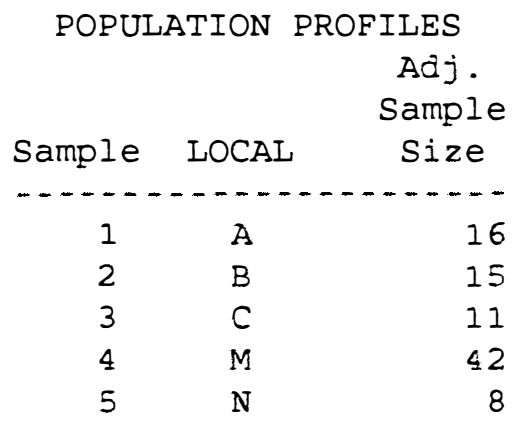

Verifica-se que, neste caso, os tamanhos das amostras foram ajustados em decorrência da utilização da opção ADDCELL = 0.5 no programa.

\begin{tabular}{crr} 
RESPONSE PROFILES \\
Response & HI & H2 \\
\hdashline 1 & -1 & 0 \\
2 & -1 & 1 \\
3 & 0 & -1 \\
4 & 0 & 0 \\
5 & 0 & 1 \\
6 & 1 & -1 \\
7 & 1 & 0 \\
8 & 1 & 1
\end{tabular}

\begin{tabular}{ccccr}
\multicolumn{5}{c}{ ADJUSTED RESPONSE FREQUENCIES } \\
Sample & 1 & 2 & 3 & 4 \\
& \multicolumn{5}{c}{ Response } & Number \\
1 & 1.5 & 5.5 & 0.5 & 1.5 \\
2 & 0.5 & 0.5 & 1.5 & 5.5 \\
3 & 0.5 & 0.5 & 0.5 & 1.5 \\
4 & 3.5 & 5.5 & 1.5 & 5.5 \\
5 & 0.5 & 0.5 & 1.5 & 1.5
\end{tabular}




\begin{tabular}{crrrr}
\multicolumn{5}{c}{ ADJUSTED RESPONSE FREQUENCIES } \\
Sample & 5 & 6 & 7 & 8 \\
\hdashline & & \multicolumn{4}{c}{ Response } & Number \\
1 & 0.5 & 0.5 & 1.5 & 4.5 \\
2 & 1.5 & 0.5 & 3.5 & 1.5 \\
3 & 2.5 & 0.5 & 3.5 & 1.5 \\
4 & 1.5 & 2.5 & 8.5 & 13.5 \\
5 & 0.5 & 0.5 & 0.5 & 2.5
\end{tabular}

Em decorrência da utilização da opção $\mathrm{ADDCELL}=0.5$ no programa, as frequências das amostras foram ajustadas. Todas as análises posteriores são efetuadas baseadas nestas frequências ajustadas.

\begin{tabular}{lcrr}
\multicolumn{4}{c}{ ANALYSIS-OF-VARIANCE TABLE } \\
SOURCe & DF & Chi-Square & Prob \\
\hline INTERCEPTO COMP & 1 & 93.81 & 0.0000 \\
LOCAL COMP & 2 & 2.92 & 0.2319 \\
CULTIVAR COMP & 1 & 2.25 & 0.1335 \\
LOCAL X CULTIVAR COMP & 2 & 0.95 & 0.6211 \\
INTERCEPTO INC & 1 & 16.31 & 0.0001 \\
LOCAL INC & 2 & 0.88 & 0.6441 \\
CULTIVAR INC & 1 & 0.39 & 0.5348 \\
LOCAL X CULTIVAR INC & 2 & 0.31 & 0.8560 \\
& & & \\
RESIDUAL & 0 &. &.
\end{tabular}

Observa-se que alguns valores da tabela da análise de variância estão incoerentes. Caso fosse de interesse, poder-se-ia construir contrastes especificos para a obtenção de valores coerentes. Mas, neste caso, estes valores em nada invalidam as análises posteriores. Por este motivo, não foram construidos contrastes específicos. 


\begin{tabular}{|c|c|c|c|c|c|}
\hline Effect & Parameter & Estimate & $\begin{array}{c}\text { Standard } \\
\text { Error }\end{array}$ & $\begin{array}{l}\text { Chi- } \\
\text { square }\end{array}$ & Prob \\
\hline & $\cdots$ & - & & 070 & \\
\hline \multirow{20}{*}{ MODEL } & 1 & 0.5518 & 0.0570 & 93.81 & 0.0000 \\
\hline & 2 & 0.1357 & 0.1211 & 1.26 & 0.2624 \\
\hline & 3 & -0.2184 & 0.0956 & 5.21 & 0.0224 \\
\hline & 4 & -0.0518 & 0.0938 & 0.30 & 0.5812 \\
\hline & 5 & 0.0862 & 0.0790 & 1.19 & 0.2753 \\
\hline & 6 & 0.0644 & 0.0429 & 2.25 & 0.1335 \\
\hline & 7 & -0.00192 & 0.0790 & 0.00 & 0.9806 \\
\hline & 8 & 0.0189 & 0.0819 & 0.05 & 0.8173 \\
\hline & 9 & -0.00886 & 0.1129 & 0.01 & 0.9375 \\
\hline & 10 & 0.0563 & 0.0577 & 0.95 & 0.3291 \\
\hline & 11 & 0.5022 & 0.1244 & 16.31 & 0.0001 \\
\hline & 12 & 0.1407 & 0.2379 & 0.35 & 0.5542 \\
\hline & 13 & -0.1272 & 0.2589 & 0.24 & 0.6233 \\
\hline & 14 & -0.00218 & 0.3008 & 0.00 & 0.9942 \\
\hline & 15 & 0.1159 & 0.1680 & 0.48 & 0.4903 \\
\hline & 16 & -0.0772 & 0.1244 & 0.39 & 0.5348 \\
\hline & 17 & -0.0657 & 0.2379 & 0.08 & 0.7825 \\
\hline & 18 & -0.0478 & 0.2589 & 0.03 & 0.8535 \\
\hline & 19 & 0.0772 & 0.3008 & 0.07 & 0.7975 \\
\hline & 20 & 0.0841 & 0.1680 & 0.25 & 0.6165 \\
\hline
\end{tabular}

ANALYSIS OF CONTRASTS

$\begin{array}{llrr}\text { Contrast } & \text { DF } & \text { Chi-Square } & \text { Prob } \\ \text { BETAC VS BETAI } & 4 & 1.88 & 0.7582\end{array}$

Verifica-se que não há diferença estatisticamente significativa entre os parâmetros do modelo com dados completos e aqueles do modelo com dados incompletos (BETAC VS BETAI). Desta forma, a análise incluindo os dados incompletos pode ser implementada sem maiores riscos. 


\begin{tabular}{|c|c|c|c|c|c|}
\hline & & PREDICTED & VAIUES FOR & RESPONSE FUN & TIONS \\
\hline & Function & -....-Obse & $\begin{array}{c}\text { cved-....... } \\
\text { Standard }\end{array}$ & -..--Pred & $\begin{array}{l}\text { cted........ } \\
\text { Standard }\end{array}$ \\
\hline Sample & Number & Function & Error & Function & Error \\
\hline$\ldots$ & - & $\ldots-\ldots$ & $\ldots$ & $\ldots$ & $\ldots$ \\
\hline 1 & 1 & 0.75 & 0.15309311 & 0.75 & 0.15309311 \\
\hline & 2 & 0.625 & 0.1711633 & 0.625 & 0.1711633 \\
\hline & 3 & 0.5 & 0.5 & 0.5 & 0.5 \\
\hline & 4 & 0.78571429 & 0.15508861 & 0.78571429 & 0.15508861 \\
\hline 2 & 1 & 0.41666667 & 0.14231876 & 0.41666667 & 0.14231876 \\
\hline & 2 & 0.25 & 0.125 & 0.25 & 0.125 \\
\hline & 3 & 0.25 & 0.30618622 & 0.25 & 0.30618622 \\
\hline & 4 & 0.5 & 0.5 & 0.5 & 0.5 \\
\hline 3 & 1 & 0.55555556 & 0.16563466 & 0.55555556 & 0.16563466 \\
\hline & 2 & 0.44444444 & 0.16563466 & 0.44444444 & 0.16563466 \\
\hline & 3 & 0.5 & 0.5 & 0.5 & 0.5 \\
\hline & 4 & 0.5 & 0.5 & 0.5 & 0.5 \\
\hline 4 & 1 & 0.75862069 & 0.07946271 & 0.75862069 & 0.07946271 \\
\hline & 2 & 0.51724138 & 0.09279245 & 0.51724138 & 0.09279245 \\
\hline & 3 & 0.625 & 0.24206146 & 0.625 & 0.24206146 \\
\hline & 4 & 0.61111111 & 0.16249934 & 0.61111111 & 0.16249934 \\
\hline 5 & 1 & 0.6 & 0.21908902 & 0.6 & 0.21908902 \\
\hline & 2 & 0.6 & 0.21908902 & 0.6 & 0.21908902 \\
\hline & 3 & 0.25 & 0.30618622 & 0.25 & 0.30618622 \\
\hline & 4 & 0.5 & 0.5 & 0.5 & 0.5 \\
\hline
\end{tabular}




\section{CONCLUSÕES}

Do exposto anteriormente pode-se concluir que:

- em alguns casos o modelo alternativo, que inclui locais em vez de anos, pode ser mais informativo que o modelo de Eskridge (1997);

- o teste do mecanismo de dados incompletos de Park \& Davis (1993) é dependente do modelo, conforme foi verificado pela análise do segundo conjunto de dados. A rigor, o teste serve para verificar se os parâmetros do modelo com dados completos não diferem estatisticamente dos parâmetros do modelo com dados incompletos. Desta forma, o teste não verifica a estrutura propriamente dita dos dados, não sendo útil, portanto, para verificar a condição de MCAR. 


\section{REFERÊNCIAS BIBLIOGRÁFICAS}

ÁGRESTI, A. Categorical Data Analysis. New York: John Wiley, 1990.

ESKRIDGE, K. M.; MUMM, R. F. Choosing cultivars based on the probability of outperforming a check. Theoretical and Applied Genetics, v.87, p.494-500.

ESKRIDGE, K. M. Evaluation of com hybrids using the probability of outperforming a check based on strip-test data. Journal of Agricultural, Biological and Environmental Statistics, v.2, p.245-254, 1997.

FONSECA, F. C. E. Teste do mecanismo de dados incompletos para medidas repetidas categorizadas. Piracicaba, 1997. 30p. Seminários em Estatística e Experimentação Agronômica - Departamento de Matemática e Estatística/Escola Superior de Agricultura "Luiz de Queiroz", Universidade de São Paulo.

GRIZZLE, J. E; STARMER, C. F.; KOCH, G. G. Analysis of categorical data by linear models. Biometrics, v.25, p.489-504, 1969.

KOCH, G. G.; MMREY, P. B.; REINFURT, D. W. Linear model analysis of categorical data with incomplete response vectors. Biometrics, v.28, p.663-692, 1972.

KOCH, G. G.; LANDIS, J. R.; FREEMAN, J. L.; FREEMAN, D. HH.; LEHNEN, R. G. A general methodology for the analysis of experiments with repeated measurement of categorical data. Biometrics, v.33, p.133-158, 1977.

LAIRD, N. M. Missing data in longitudinal studies. Statistics in Medicine, v.7, p.305315, 1988.

LIPSITZ, S. R.; LAIRD, N. M.; HARRINGTON, D. P. Weighted least squares analysis of repeated categorical measurement with outcomes subject to nonresponse. Biometrics, v.50, p.11-24, 1994. 
LITTLE, R. J. A. A test of missing completely at random for multivariate data with missing values. Journal of the American Statistical Association, v.83, p.1198-1202, 1988.

LITTLE, R. J. A.; RUBIN, D. B. Statistical Analysis With Missing Data. New York: John Wiley, 1987.

OGLIARI, P. J. Análise de dados categorizados: modelos log-lineares e independência em tabelas de contingência. Piracicaba, 1984. 96p. Dissertação (Mestrado) - Escola Superior de Agricultura "Luiz de Queiroz", Universidade de São Paulo.

PARK, T.; DAVIS, C.S. A test of the missing data mechanism for repeated categorical data. Biometrics, v.49, p.631-638, 1993.

SAS INSTITUTE. SAS/STAT User's Guide. 4.ed. North Carolina: SAS Institute, 1990.

SEGALA, L. F. Análise de experimentos com medidas repetidas com respostas categóricas incompletas. São José do Rio Preto, 1997. 74p. Dissertação (Mestrado) Instituto de Biociências, Letras e Ciências Exatas, Universidade Estadual Paulista.

WOOLSON, R. F.; CLARKE, W. R. Analysis of categorical incomplete longitudinal data. Journal of the Royal Statistical Society, v.147, p.87-99, 1984. 